\title{
Horienoids A and B, Two Heterocoupled Sesquiterpenoid Dimers from
}

\section{Hedyosmum orientale}

Yao-Yue Fan, ${ }^{\dagger}$ Li-She Gan, ${ }^{\S, \#}$ Shi-Xin Chen,${ }^{\S}$ Qi Gong, ${ }^{\star}$ Hai-Yan Zhang, ${ }^{\star}$ and Jian-Min Yue ${ }^{*} \dagger$

${ }^{\dagger}$ State Key Laboratory of Drug Research, Shanghai Institute of Materia Medica, Chinese Academy of Sciences, Shanghai 201203, China

†CAS Key Laboratory of Receptor Research, Shanghai Institute of Materia Medica, Chinese Academy of Sciences, Shanghai 201203, China

${ }^{\S}$ Institute of Modern Chinese Medicine, College of Pharmaceutical Sciences, Zhejiang University, Hangzhou 310058, China

${ }^{\#}$ School of Biotechnology and Health Sciences, Wuyi University, Jiangmen 529020, China 


\section{Table of contents}

Table S1. ${ }^{1} \mathrm{H}(500 \mathrm{~Hz})$ and ${ }^{13} \mathrm{C}\left\{{ }^{1} \mathrm{H}\right\}(125 \mathrm{~Hz})$ NMR Data for Compound 1...............................S4

Table S2. DP4+ evaluation of theoretical and experimental data...........................................S5

Table S3. Inhibitory effect of positive control on LPS-induced BV-2 cell inflammation........S6

Table S4. Inhibitory effect of compound 2 on LPS-induced BV-2 cell inflammation.............S6

Scheme S1. Hypothetical Biosynthetic Pathway of Compound 2................................................ 7

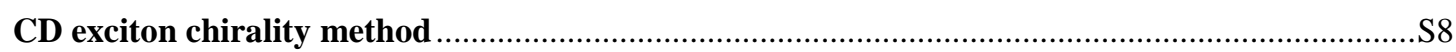

Figure S1. CD spectra of $\mathbf{1}$ and $\mathbf{2}$ (in $\mathrm{MeOH}$ ). The stereoview of $\mathbf{1}$ and $\mathbf{2}$ : arrows denote the electric

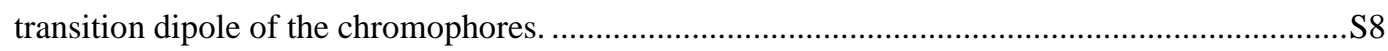

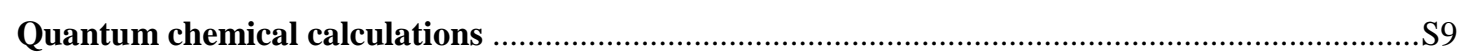

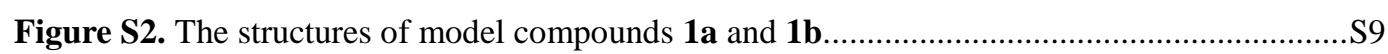

Figure S3. B3LYP/6-31G(d) optimized lowest energy conformers for 1a..................................S9

Figure S4. B3LYP/6-31G(d) optimized lowest energy conformers for $\mathbf{1 b}$..................................S9

Figure S5. B3LYP/6-31G(d) optimized lowest energy conformers for 2. .................................S10

Table S5. Energy (298.15 K) analysis for 1a, 1b, and 2. .................................................. 11

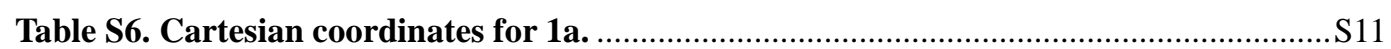

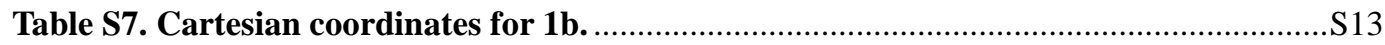

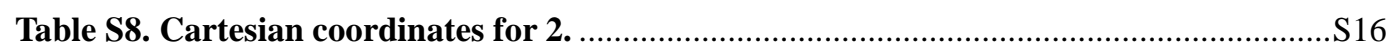

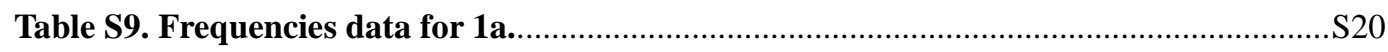

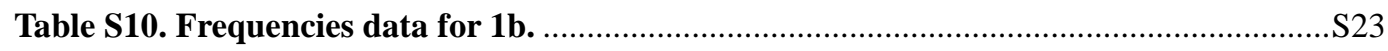

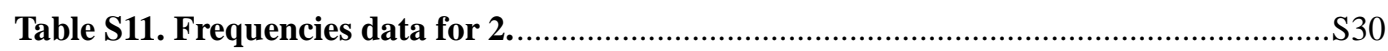

Table S12. Calculated ${ }^{1} \mathrm{H}$-NMR chemical shifts for conformers of 1a and 1b.....................S33

Table S13. Calculated ${ }^{13} \mathbf{C}\left\{{ }^{1} \mathbf{H}\right\}$-NMR chemical shifts for conformers of $1 \mathrm{a}$ and $\mathbf{1 b}$.............S35

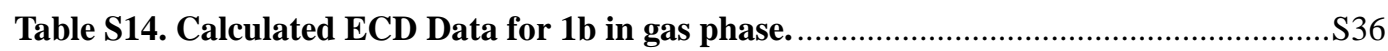

Table S15. Calculated ECD Data for 2 in gas phase. .........................................................S38

Calculated Specific OR Data for Compounds 1 and 2. ..................................................... 440

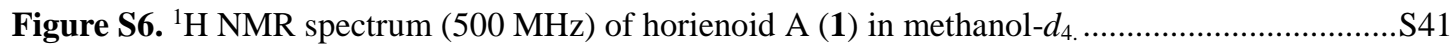

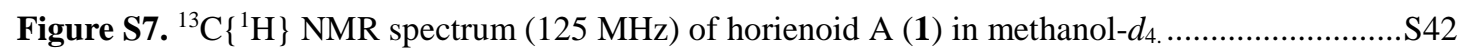

Figure S8. ${ }^{1} \mathrm{H}-{ }^{1} \mathrm{H}$ COSY spectrum $(500 \mathrm{MHz})$ of horienoid A (1) in methanol- $d_{4} \ldots \ldots \ldots \ldots \ldots \ldots \ldots \ldots \ldots . . . . . . . . . . . . . . .343$

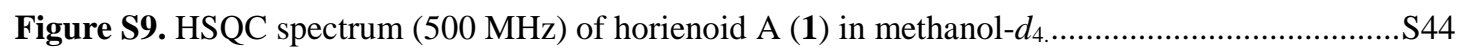

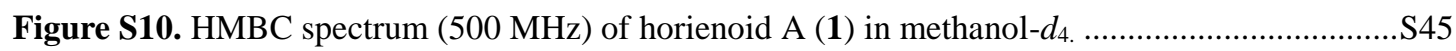

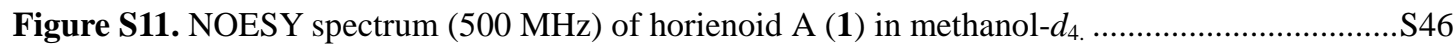

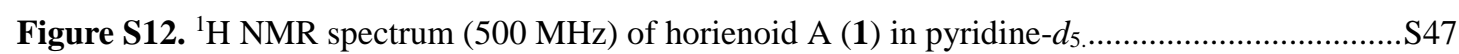

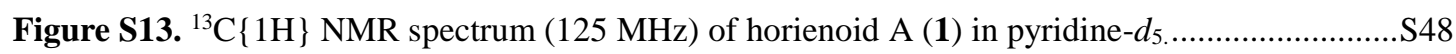

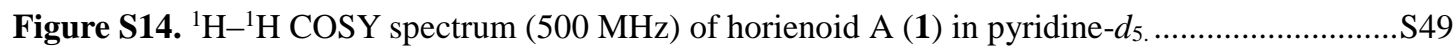

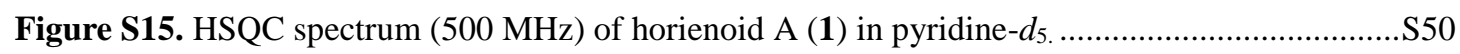

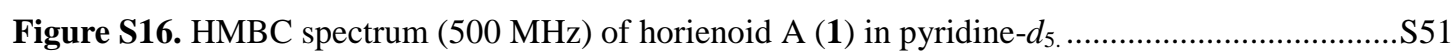

Figure S17. NOESY spectrum (500 MHz) of horienoid A (1) in pyridine- $d_{5} \ldots \ldots \ldots \ldots \ldots \ldots \ldots \ldots \ldots \ldots \ldots . . . . .552$

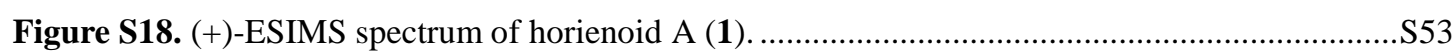

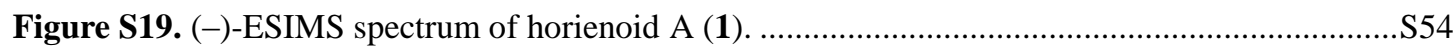

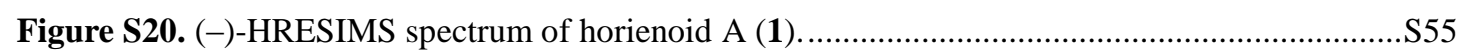


Figure S21. IR spectrum of horienoid A (1). S56

Figure S22. ${ }^{1} \mathrm{H}$ NMR spectrum $(500 \mathrm{MHz})$ of horienoid B (2) in methanol- $d_{4}$..............................S57

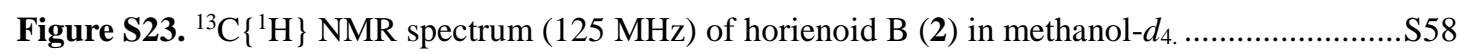

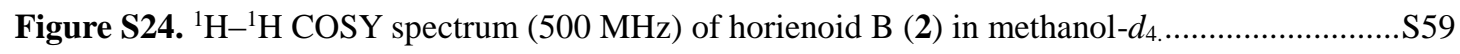

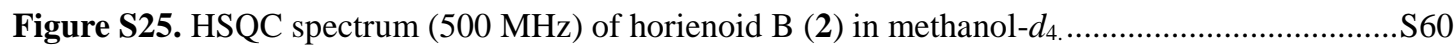

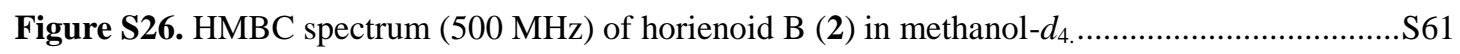

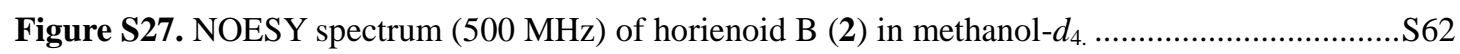

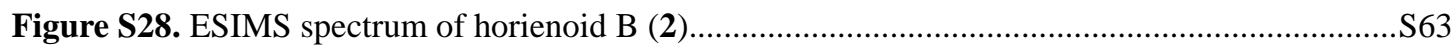

Figure S29. (+)-HRESIMS spectrum of horienoid B (2) ..........................................................S64

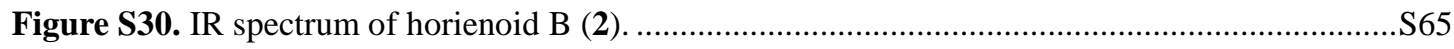


Table S1. ${ }^{1} \mathrm{H}(500 \mathrm{~Hz})$ and ${ }^{13} \mathrm{C}\left\{{ }^{1} \mathrm{H}\right\}(125 \mathrm{~Hz})$ NMR Data for Compound 1.

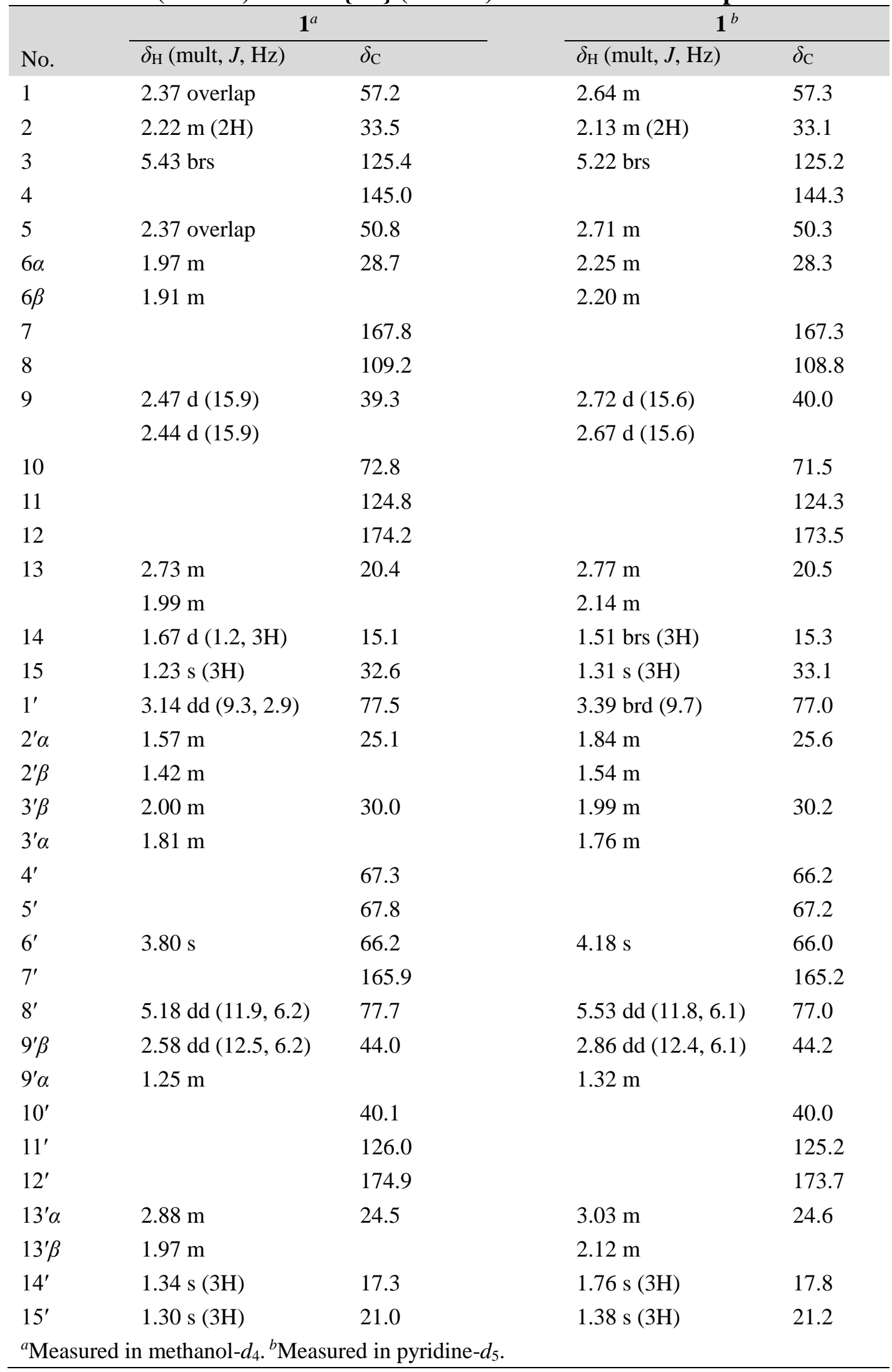


Table S2. DP4+ evaluation of theoretical and experimental data.

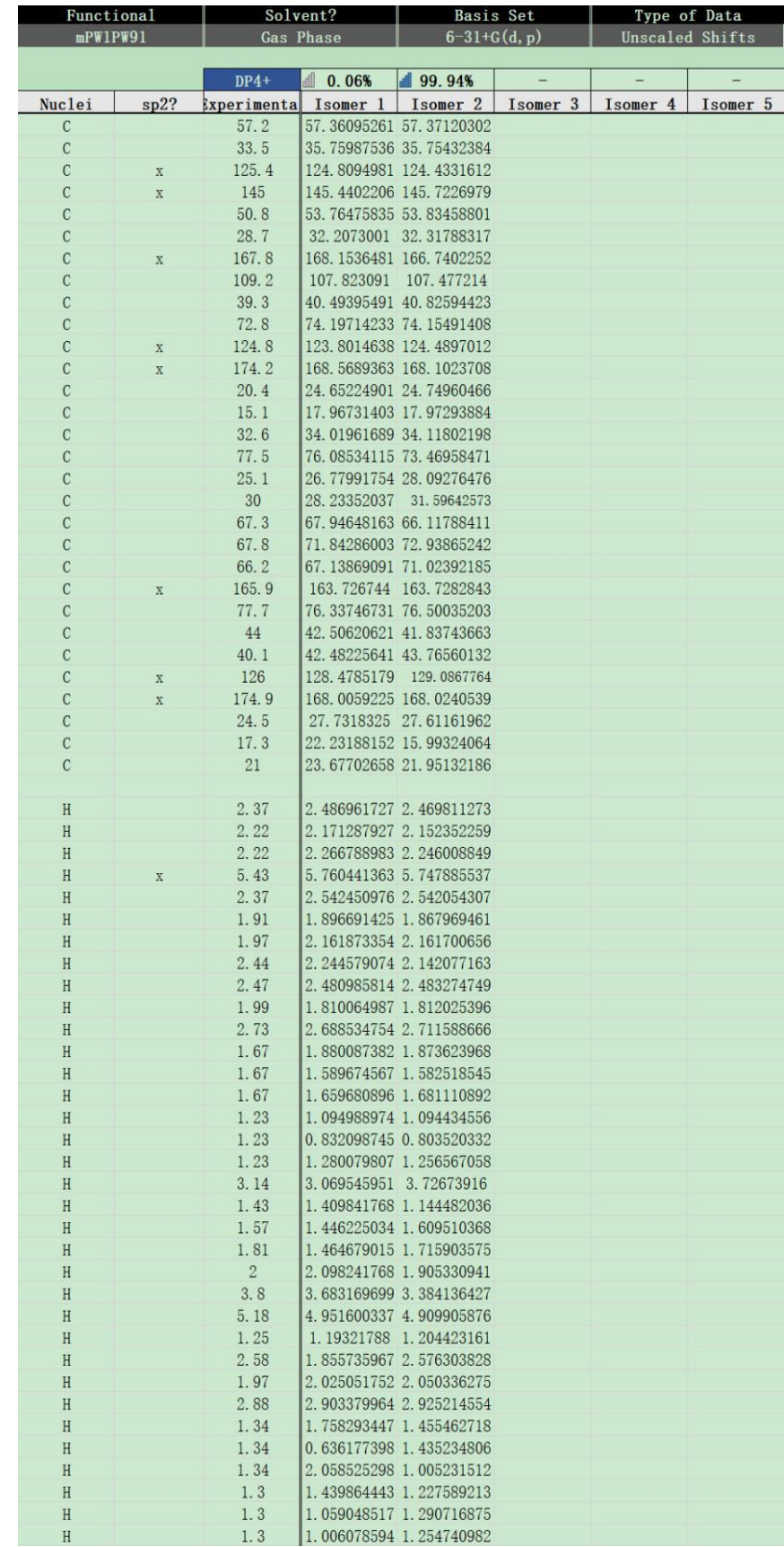

\begin{tabular}{|c|c|c|c|c|c|c|}
\hline Functional & \multicolumn{2}{|c|}{ Solvent? } & \multicolumn{2}{|c|}{ Basis Set } & \multicolumn{2}{|c|}{ Type of Data } \\
\hline $\mathrm{mPW} 1 \mathrm{PW} 91$ & Gas & Phase & $6-31+$ & $(d, p)$ & Unscale & Shifts \\
\hline & Isomer 1 & Isomer 2 & Isomer 3 & Isomer 4 & Isomer 5 & Isomer 6 \\
\hline sDP4+ (H data) & $0.00 \%$ & $100.00 \%$ & - & - & - & - \\
\hline sDP4+ (C data) & $-199.99 \%$ & (ती $0.01 \%$ & - & - & - & - \\
\hline sDP4+ (a11 data) & Hil $1.50 \%$ & - $98.50 \%$ & - & - & - & - \\
\hline uDP4+ (H data) & $0.01 \%$ & $99.99 \%$ & - & - & - & - \\
\hline uDP4+ (C data) & $-199.69 \%$ & $0.31 \%$ & - & - & - & - \\
\hline uDP4+ (a11 data) & $3.96 \%$ & $\begin{array}{r}96.04 \% \\
\end{array}$ & - & - & - & - \\
\hline DP4+ (H data) & (1il) $0.00 \%$ & $100.00 \%$ & - & - & - & - \\
\hline DP4+ (C data $)$ & $100.00 \%$ & $0.00 \%$ & - & - & - & - \\
\hline DP4+ (a11 data) & 메 $0.06 \%$ & - $99.94 \%$ & - & - & - & - \\
\hline
\end{tabular}


Table S3. Inhibitory effect of positive control on LPS-induced BV-2 cell inflammation.

\begin{tabular}{|c|c|c|}
\hline Groups & $\begin{array}{c}\text { Cell viability } \\
\text { ( \% of control group ) }\end{array}$ & $\begin{array}{c}\text { NO production } \pm \text { SEM } \\
(\% \text { of LPS group ) }\end{array}$ \\
\hline Control & 100.0 & $10.38 \% \pm 1.44$ \\
\hline Model - LPS (100 ng/ml) & - & $100.0 \% \pm 0.00$ \\
\hline $\begin{array}{c}\text { Positive control - } \\
\text { Resveratrol ( } 20 \mu \mathrm{M})\end{array}$ & 72.88 & $63.32 \% \pm 1.53^{* * *}$ \\
\hline
\end{tabular}

Table S4. Inhibitory effect of compound 2 on LPS-induced BV-2 cell inflammation.

\begin{tabular}{|c|c|c|c|c|}
\hline \multirow{2}{*}{ compound } & \multicolumn{2}{|c|}{$\begin{array}{c}\text { Cell viability } \\
\text { ( \% of control group ) }\end{array}$} & \multicolumn{2}{|c|}{$\begin{array}{c}\text { NO production } \pm \text { SEM } \\
(\% \text { of LPS group })\end{array}$} \\
\hline & $1 \mu \mathrm{M}$ & $10 \mu \mathrm{M}$ & $1 \mu \mathrm{M}$ & $10 \mu \mathrm{M}$ \\
\hline 2 & $114.7 \%$ & $97.14 \%$ & $93.90 \% \pm 1.64^{*}$ & $63.62 \% \pm 1.96^{* * *}$ \\
\hline
\end{tabular}

${ }^{*} \mathrm{P}<0.05,{ }^{* * *} \mathrm{P}<0.001$ vs model group. 
Scheme S1. Hypothetical Biosynthetic Pathway of Compound 2.

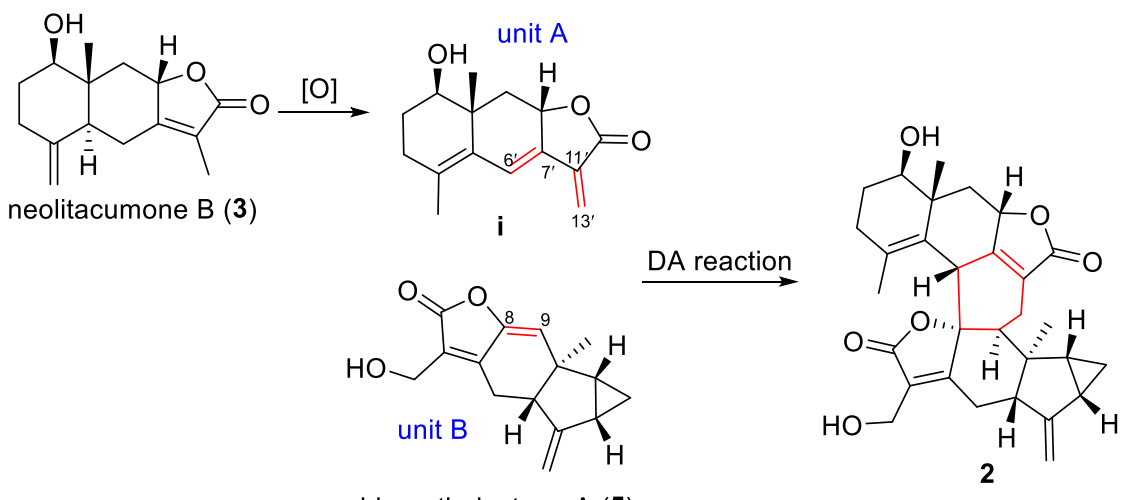




\section{CD exciton chirality method}

The absolute configuration of $\mathbf{1}$ was also determined by biosynthetic consideration and exciton chiral method of electric circular dichroism (ECD). Consistent with its UV maximum absorption at $228 \mathrm{~nm}(\log \varepsilon 4.14)$ ascribed to two $\alpha, \beta$-unsaturated butyrolactone moieties, the ECD spectrum of 1 (Figure S1A) exhibited a positive Cotton effect at $220 \mathrm{~nm}(\Delta \varepsilon+41.4)$ and a negative Cotton effect at $242 \mathrm{~nm}(\Delta \varepsilon-16.1)$ due to the transition interaction between two identical chromophores. The negative chirality suggested that the transition dipole moments of the two chromophores were oriented in a counterclockwise manner (Figure S1A) and established the absolute configuration of $\mathbf{1}$ as depicted. Similarly, the absolute configuration of $\mathbf{2}$ was determined by ECD exciton chirality method (Figure S1B), in which a positive Cotton effect at $222 \mathrm{~nm}(\Delta \varepsilon+16.8)$ and a negative Cotton effect at $242 \mathrm{~nm}(\Delta \varepsilon-22.9)$ uncovered the negative chirality and determined the absolute configuration of $\mathbf{2}$ as shown.
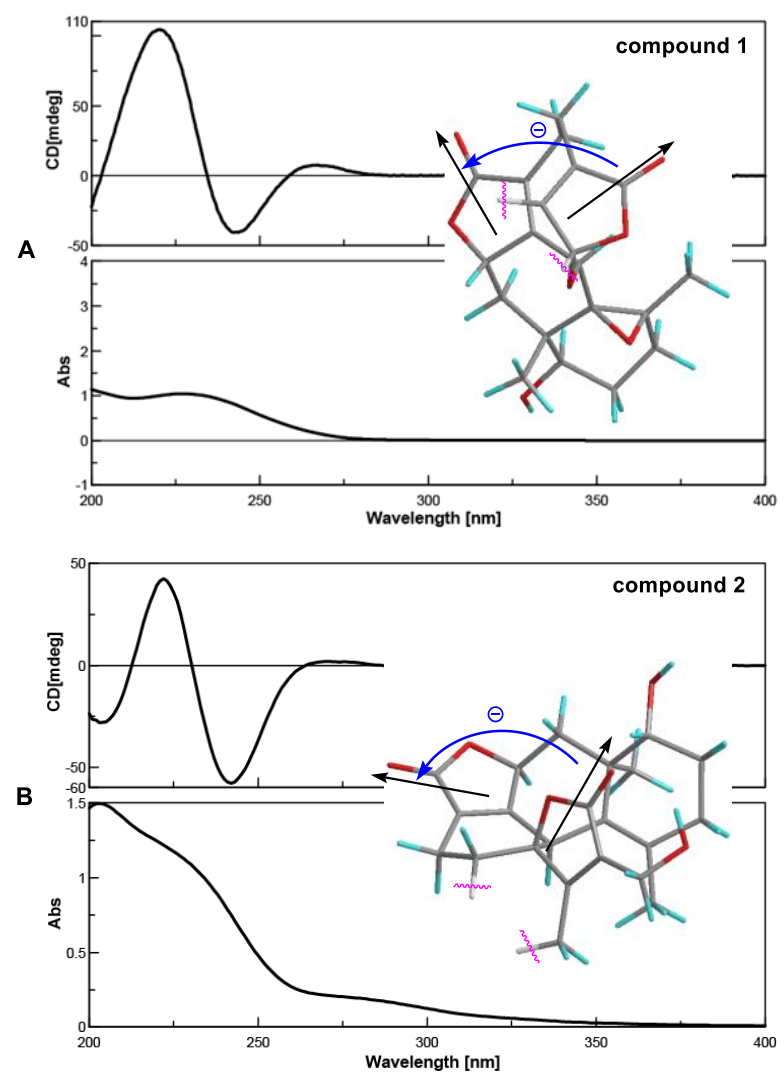

Figure S1. CD spectra of $\mathbf{1}$ and $\mathbf{2}$ (in $\mathrm{MeOH}$ ). The stereoview of $\mathbf{1}$ and 2: arrows denote the electric transition dipole of the chromophores. 


\section{Quantum chemical calculations}
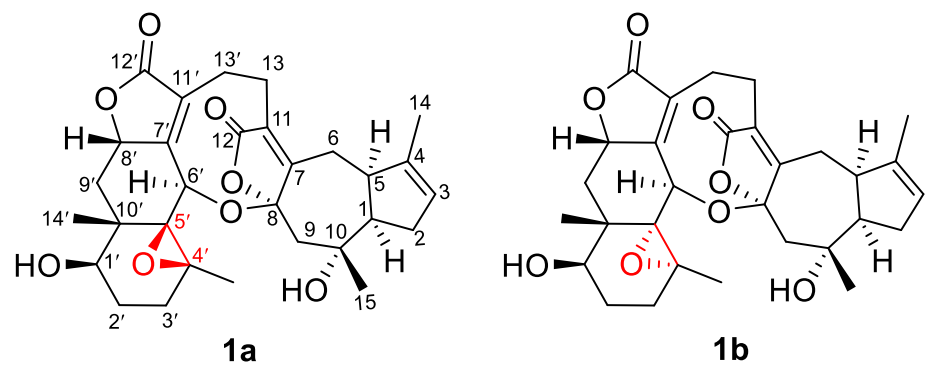

Figure S2. The structures of model compounds $\mathbf{1 a}$ and $\mathbf{1 b .}$

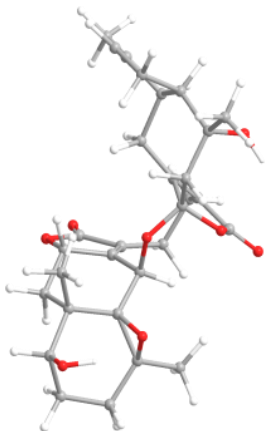

C1

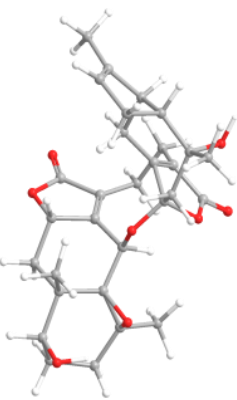

C2

Figure S3. B3LYP/6-31G(d) optimized lowest energy conformers for 1a.

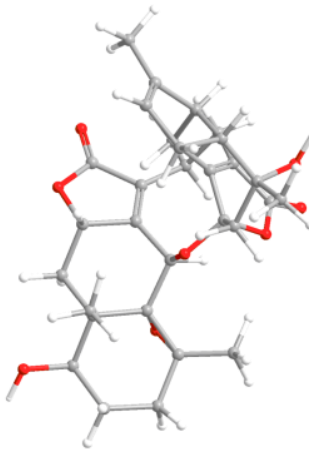

C1

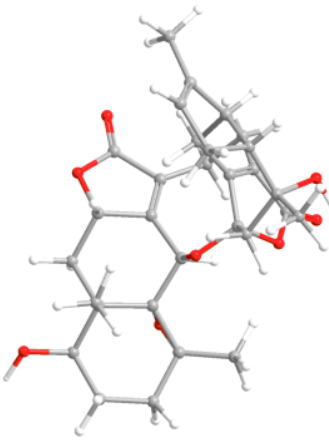

C2

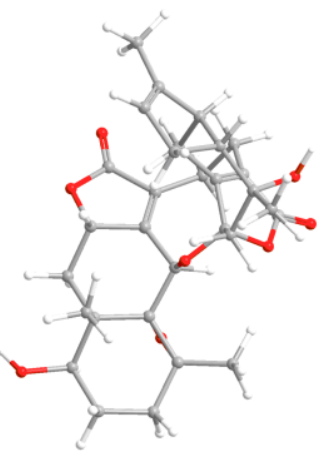

C3

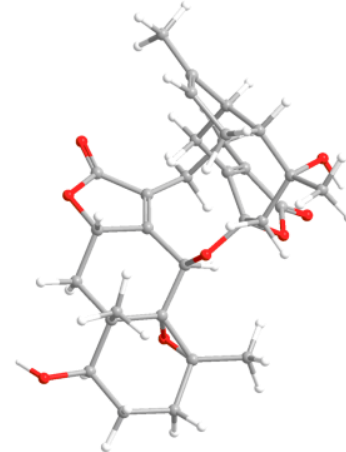

C4

Figure S4. B3LYP/6-31G(d) optimized lowest energy conformers for $\mathbf{1 b}$. 


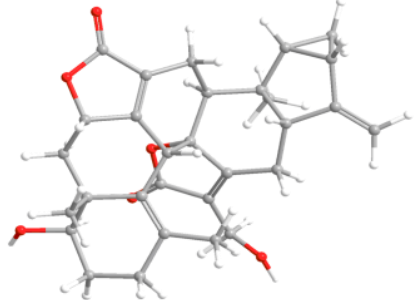

C1

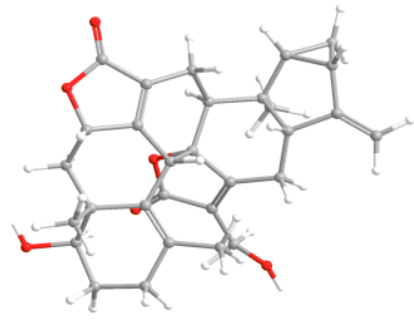

C2

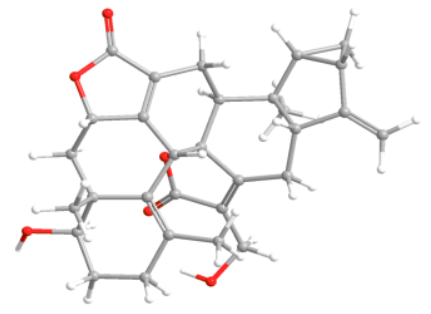

C3

Figure S5. B3LYP/6-31G(d) optimized lowest energy conformers for 2. 
Table S5. Energy(298.15 K) analysis for 1a, 1b, and 2.

\begin{tabular}{|l|l|l|l|l|l|}
\hline Conf. & \multicolumn{1}{|c|}{ G (Hartree) } & \multicolumn{1}{|c|}{ E(Hartree $)$} & $\Delta \mathrm{G}(\mathrm{Kcal} / \mathrm{mol})$ & Boltzmann Distribution \\
\hline \multicolumn{5}{|c|}{$\mathbf{1 b}$} \\
\hline C1 & -1766.044535 & -1765.951078 & 0 & 0.958164863 \\
\hline C2 & -1766.04158 & -1765.948037 & 1.85429205 & 0.041835137 \\
\hline \multicolumn{5}{|c|}{} \\
\hline C1 & -1766.042623 & -1765.947657 & 1.80534627 & 0.025561098 \\
\hline C2 & -1766.0455 & -1765.950640 & 0 & 0.538992511 \\
\hline C3 & -1766.042377 & -1765.947460 & 1.95971373 & 0.019695619 \\
\hline C4 & -1766.045255 & -1765.950443 & 0.15373995 & 0.415750772 \\
\hline & -1614.369179 & -1614.278454 & 1.2236445 & 0.097453447 \\
\hline C1 & -1614.369473 & -1614.279623 & 1.03915656 & 0.133075101 \\
\hline C2 & -1614.371129 & -1614.282262 & 0 & 0.769471452 \\
\hline C3 & & $\mathbf{2}$ & \\
\hline
\end{tabular}

Table S6. Cartesian coordinates for $1 \mathrm{a}$.

\begin{tabular}{|c|c|c|c|c|c|c|}
\hline & \multicolumn{3}{|c|}{ C1 } & \multicolumn{3}{|c|}{$\mathrm{C} 2$} \\
\hline $\mathrm{O}$ & 0.174262 & -1.01949 & -0.12279 & 0.155438 & -1.02538 & -0.08711 \\
\hline $\mathrm{C}$ & -1.62261 & 0.285255 & 0.769937 & -1.63012 & 0.279943 & 0.816582 \\
\hline $\mathrm{C}$ & -1.04662 & -1.10515 & 0.633232 & -1.05001 & -1.11158 & 0.703839 \\
\hline $\mathrm{C}$ & -1.31469 & 0.753531 & 1.991988 & -1.32455 & 0.766781 & 2.030545 \\
\hline $\mathrm{C}$ & -0.80891 & -0.3844 & 2.794648 & -0.79577 & -0.35676 & 2.844671 \\
\hline $\mathrm{O}$ & -0.75311 & -1.50684 & 1.979749 & -0.71348 & -1.4776 & 2.04283 \\
\hline $\mathrm{O}$ & -0.47802 & -0.42172 & 3.954228 & -0.45131 & -0.36443 & 4.001367 \\
\hline $\mathrm{C}$ & -1.1158 & 2.158197 & 2.450421 & -1.12247 & 2.178413 & 2.465918 \\
\hline $\mathrm{C}$ & 0.398936 & 2.519599 & 2.243558 & 0.391491 & 2.534262 & 2.243059 \\
\hline $\mathrm{C}$ & 0.850657 & 2.354467 & 0.821645 & 0.832627 & 2.356675 & 0.819016 \\
\hline $\mathrm{C}$ & 1.103585 & 1.263262 & 0.070501 & 1.084953 & 1.260252 & 0.07485 \\
\hline $\mathrm{C}$ & 1.227869 & -0.20008 & 0.429023 & 1.215462 & -0.20098 & 0.442634 \\
\hline $\mathrm{C}$ & -1.88315 & -2.166 & -0.07187 & -1.88751 & -2.20027 & 0.036442 \\
\hline $\mathrm{C}$ & -3.40554 & -2.09765 & 0.200102 & -3.41552 & -2.09441 & 0.218139 \\
\hline $\mathrm{C}$ & -4.17146 & -1.01791 & -0.60307 & -4.12511 & -1.02306 & -0.65422 \\
\hline $\mathrm{C}$ & -3.83206 & 0.498543 & -0.42852 & -3.80158 & 0.494198 & -0.46975 \\
\hline $\mathrm{C}$ & -2.3224 & 0.905264 & -0.38637 & -2.29696 & 0.894868 & -0.36349 \\
\hline $\mathrm{C}$ & 1.014405 & 3.549553 & -0.05635 & 0.984879 & 3.545191 & -0.06974 \\
\hline $\mathrm{O}$ & 1.39702 & 3.127446 & -1.3065 & 1.360811 & 3.115189 & -1.31922 \\
\hline $\mathrm{C}$ & 1.441457 & 1.693007 & -1.33137 & 1.409952 & 1.680694 & -1.33288 \\
\hline $\mathrm{C}$ & 2.816291 & 1.16757 & -1.7537 & 2.782184 & 1.155701 & -1.76335 \\
\hline $\mathrm{C}$ & 2.915366 & -0.37289 & -1.57304 & 2.884356 & -0.38355 & -1.57487 \\
\hline $\mathrm{C}$ & 2.574669 & -0.72713 & -0.10425 & 2.557363 & -0.7301 & -0.10131 \\
\hline
\end{tabular}




\begin{tabular}{|c|c|c|c|c|c|c|}
\hline $\mathrm{C}$ & 4.364055 & -0.84725 & -1.92391 & 4.329928 & -0.85876 & -1.93701 \\
\hline $\mathrm{C}$ & 5.399213 & -0.37873 & -0.89708 & 5.374112 & -0.38397 & -0.92217 \\
\hline $\mathrm{C}$ & 5.128153 & -0.97079 & 0.491601 & 5.116536 & -0.96933 & 0.471982 \\
\hline $\mathrm{C}$ & 3.654918 & -1.02471 & 0.893307 & 3.647094 & -1.02314 & 0.88731 \\
\hline $\mathrm{C}$ & -4.26381 & -1.21562 & -2.13853 & -4.12307 & -1.22677 & -2.19296 \\
\hline $\mathrm{C}$ & -4.67005 & 0.160632 & -2.60676 & -4.52042 & 0.143471 & -2.6876 \\
\hline $\mathrm{C}$ & -4.47522 & 1.101531 & -1.67423 & -4.38692 & 1.08863 & -1.74827 \\
\hline $\mathrm{O}$ & 0.864254 & 4.714244 & 0.222539 & 0.830933 & 4.711702 & 0.200246 \\
\hline $\mathrm{C}$ & -4.7675 & 2.567658 & -1.79259 & -4.68402 & 2.551936 & -1.88637 \\
\hline $\mathrm{C}$ & 3.447247 & -0.93174 & 2.395738 & 3.452083 & -0.92525 & 2.391104 \\
\hline $\mathrm{O}$ & 4.441496 & -2.25813 & -2.09913 & 4.407061 & -2.27057 & -2.10516 \\
\hline $\mathrm{C}$ & 1.947353 & -1.07997 & -2.55221 & 1.906874 & -1.09701 & -2.53984 \\
\hline $\mathrm{H}$ & 0.674142 & 1.363941 & -2.04238 & 0.637828 & 1.343834 & -2.03502 \\
\hline $\mathrm{O}$ & 2.882613 & -2.0852 & 0.26115 & 2.870098 & -2.08652 & 0.266217 \\
\hline $\mathrm{H}$ & 1.223741 & -0.31144 & 1.509364 & 1.224587 & -0.3027 & 1.523633 \\
\hline $\mathrm{C}$ & -4.01639 & -3.47963 & -0.09507 & -4.0315 & -3.47465 & -0.07566 \\
\hline $\mathrm{O}$ & -3.65743 & -1.75872 & 1.573806 & -3.61783 & -1.77542 & 1.604566 \\
\hline $\mathrm{H}$ & -5.20102 & -1.09912 & -0.23108 & -5.18532 & -1.1005 & -0.36468 \\
\hline $\mathrm{H}$ & -4.28528 & 0.874982 & 0.496235 & -4.29697 & 0.878495 & 0.430738 \\
\hline $\mathrm{H}$ & -1.74073 & 2.858431 & 1.887915 & -1.75042 & 2.872304 & 1.898663 \\
\hline $\mathrm{H}$ & -1.34998 & 2.269623 & 3.513588 & -1.34717 & 2.304791 & 3.529507 \\
\hline $\mathrm{H}$ & 0.556387 & 3.562197 & 2.537154 & 0.553734 & 3.578963 & 2.526514 \\
\hline $\mathrm{H}$ & 0.987648 & 1.889843 & 2.919566 & 0.983146 & 1.908414 & 2.919918 \\
\hline $\mathrm{H}$ & -1.48809 & -3.13901 & 0.242765 & -1.53932 & -3.15472 & 0.442416 \\
\hline $\mathrm{H}$ & -1.68954 & -2.09139 & -1.14566 & -1.64773 & -2.20198 & -1.03004 \\
\hline $\mathrm{H}$ & -2.26163 & 1.997851 & -0.33679 & -2.2348 & 1.987554 & -0.31627 \\
\hline $\mathrm{H}$ & -1.84067 & 0.59735 & -1.32291 & -1.78135 & 0.583061 & -1.28051 \\
\hline $\mathrm{H}$ & 3.011706 & 1.433451 & -2.79908 & 2.968761 & 1.416129 & -2.81177 \\
\hline $\mathrm{H}$ & 3.562139 & 1.684188 & -1.14002 & 3.532256 & 1.676613 & -1.15849 \\
\hline $\mathrm{H}$ & 4.612096 & -0.42184 & -2.90441 & 4.568629 & -0.43847 & -2.92209 \\
\hline $\mathrm{H}$ & 5.404223 & 0.71636 & -0.84111 & 5.378263 & 0.711413 & -0.87176 \\
\hline $\mathrm{H}$ & 6.394046 & -0.6806 & -1.24093 & 6.366103 & -0.68638 & -1.27378 \\
\hline $\mathrm{H}$ & 5.67494 & -0.39764 & 1.249051 & 5.669307 & -0.39145 & 1.221487 \\
\hline $\mathrm{H}$ & 5.51283 & -1.99708 & 0.551378 & 5.50339 & -1.99471 & 0.533497 \\
\hline $\mathrm{H}$ & -4.99067 & -1.99006 & -2.41114 & -4.81951 & -2.01348 & -2.50654 \\
\hline $\mathrm{H}$ & -3.30586 & -1.52198 & -2.589 & -3.13394 & -1.51883 & -2.58001 \\
\hline $\mathrm{H}$ & -5.0688 & 0.349641 & -3.60045 & -4.8642 & 0.325766 & -3.70283 \\
\hline $\mathrm{H}$ & -5.21165 & 2.808664 & -2.76365 & -5.07645 & 2.78721 & -2.88079 \\
\hline $\mathrm{H}$ & -5.46428 & 2.896159 & -1.00935 & -5.42471 & 2.87754 & -1.14313 \\
\hline $\mathrm{H}$ & -3.86121 & 3.17812 & -1.68038 & -3.78956 & 3.169027 & -1.72658 \\
\hline $\mathrm{H}$ & 4.187844 & -1.56625 & 2.896711 & 4.198132 & -1.5567 & 2.887916 \\
\hline $\mathrm{H}$ & 3.600458 & 0.094284 & 2.751545 & 3.60632 & 0.102279 & 2.742152 \\
\hline $\mathrm{H}$ & 2.46059 & -1.2769 & 2.709792 & 2.468661 & -1.27123 & 2.714218 \\
\hline
\end{tabular}




\begin{tabular}{|c|c|c|c|c|c|c|}
\hline $\mathrm{H}$ & 4.002238 & -2.6517 & -1.32244 & 3.974443 & -2.65965 & -1.32236 \\
\hline $\mathrm{H}$ & 0.910909 & -0.76918 & -2.41657 & 0.870752 & -0.79187 & -2.38946 \\
\hline $\mathrm{H}$ & 2.249462 & -0.85653 & -3.58233 & 2.193175 & -0.87346 & -3.57452 \\
\hline $\mathrm{H}$ & 1.988472 & -2.16233 & -2.42474 & 1.955868 & -2.17889 & -2.4112 \\
\hline $\mathrm{H}$ & -5.10042 & -3.4569 & 0.055522 & -5.12544 & -3.43896 & 0.007753 \\
\hline $\mathrm{H}$ & -3.59452 & -4.22828 & 0.586456 & -3.65612 & -4.20317 & 0.649441 \\
\hline $\mathrm{H}$ & -3.81105 & -3.81053 & -1.11922 & -3.78539 & -3.82899 & -1.08253 \\
\hline $\mathrm{H}$ & -3.12495 & -2.34962 & 2.131505 & -4.57503 & -1.76152 & 1.766898 \\
\hline
\end{tabular}

Table S7. Cartesian coordinates for $1 \mathrm{~b}$.

\begin{tabular}{|l|l|l|l|l|l|l|}
\hline & \multicolumn{3}{|c}{ C1 } & \multicolumn{3}{|c|}{ C2 } \\
\hline O & 0.264436 & -0.96327 & -0.08955 & 0.282285 & -0.95596 & -0.11419 \\
\hline $\mathrm{C}$ & -1.65182 & 0.160464 & 0.808282 & -1.63962 & 0.180787 & 0.765497 \\
\hline $\mathrm{C}$ & -0.99975 & -1.18182 & 0.570654 & -0.99375 & -1.16122 & 0.513903 \\
\hline $\mathrm{C}$ & -1.47369 & 0.494522 & 2.097148 & -1.45581 & 0.505754 & 2.057259 \\
\hline $\mathrm{C}$ & -0.96216 & -0.70534 & 2.806215 & -0.96704 & -0.70292 & 2.7604 \\
\hline $\mathrm{O}$ & -0.75974 & -1.70839 & 1.877106 & -0.7954 & -1.71585 & 1.82395 \\
\hline $\mathrm{O}$ & -0.72246 & -0.85692 & 3.978557 & -0.73705 & -0.87538 & 3.931303 \\
\hline $\mathrm{C}$ & -1.38013 & 1.844263 & 2.722633 & -1.36276 & 1.850716 & 2.692752 \\
\hline $\mathrm{C}$ & 0.122658 & 2.297607 & 2.65004 & 0.140003 & 2.304706 & 2.628917 \\
\hline $\mathrm{C}$ & 0.659382 & 2.326303 & 1.249187 & 0.682861 & 2.333111 & 1.230786 \\
\hline $\mathrm{C}$ & 1.023863 & 1.346769 & 0.396871 & 1.044397 & 1.351725 & 0.37966 \\
\hline $\mathrm{C}$ & 1.228827 & -0.1369 & 0.603264 & 1.241917 & -0.13182 & 0.58943 \\
\hline $\mathrm{C}$ & -1.73154 & -2.20261 & -0.2997 & -1.72783 & -2.1545 & -0.38012 \\
\hline $\mathrm{C}$ & -3.27266 & -2.17963 & -0.23638 & -3.26825 & -2.17568 & -0.22628 \\
\hline $\mathrm{C}$ & -3.96227 & -1.02887 & -1.01876 & -4.01885 & -1.03318 & -0.9534 \\
\hline $\mathrm{C}$ & -3.72575 & 0.461179 & -0.61413 & -3.76035 & 0.460992 & -0.57217 \\
\hline $\mathrm{C}$ & -2.25407 & 0.89884 & -0.33562 & -2.27755 & 0.911531 & -0.36217 \\
\hline $\mathrm{C}$ & 0.798651 & 3.62318 & 0.526719 & 0.83331 & 3.629476 & 0.509415 \\
\hline $\mathrm{O}$ & 1.287705 & 3.37528 & -0.73174 & 1.325747 & 3.378314 & -0.74702 \\
\hline $\mathrm{C}$ & 1.434073 & 1.958085 & -0.91461 & 1.466184 & 1.96054 & -0.92931 \\
\hline $\mathrm{C}$ & 2.868244 & 1.56786 & -1.27421 & 2.901782 & 1.564244 & -1.27733 \\
\hline $\mathrm{C}$ & 3.057514 & 0.024472 & -1.2713 & 3.086641 & 0.020401 & -1.26973 \\
\hline $\mathrm{C}$ & 2.635397 & -0.52774 & 0.108632 & 2.651182 & -0.52852 & 0.107398 \\
\hline $\mathrm{C}$ & 4.566661 & -0.26678 & -1.48343 & 4.596955 & -0.27556 & -1.46771 \\
\hline $\mathrm{C}$ & 4.89078 & -1.75702 & -1.36261 & 4.915384 & -1.76676 & -1.34235 \\
\hline $\mathrm{C}$ & 4.516746 & -2.34932 & 0.007294 & 4.526773 & -2.35679 & 0.024549 \\
\hline $\mathrm{C}$ & 3.326841 & -1.69808 & 0.712533 & 3.333405 & -1.70033 & 0.718764 \\
\hline $\mathrm{C}$ & -3.83197 & -1.02659 & -2.56549 & -3.98738 & -1.04404 & -2.50397 \\
\hline $\mathrm{C}$ & -4.2562 & 0.381149 & -2.90988 & -4.41831 & 0.364331 & -2.8347 \\
\hline
\end{tabular}




\begin{tabular}{|c|c|c|c|c|c|c|}
\hline $\mathrm{C}$ & -4.59428 & 2.654384 & -1.82006 & -4.68117 & 2.648354 & -1.74831 \\
\hline $\mathrm{C}$ & 2.677159 & -2.6103 & 1.73588 & 2.671637 & -2.60894 & 1.737773 \\
\hline $\mathrm{O}$ & 4.911507 & 0.226525 & -2.7813 & 4.954638 & 0.215076 & -2.76282 \\
\hline $\mathrm{C}$ & 2.226552 & -0.62772 & -2.40169 & 2.26421 & -0.63141 & -2.40663 \\
\hline $\mathrm{H}$ & 0.747522 & 1.673829 & -1.72185 & 0.784233 & 1.679401 & -1.74141 \\
\hline $\mathrm{O}$ & 3.61133 & -0.34499 & 1.15811 & 3.617898 & -0.34789 & 1.165588 \\
\hline $\mathrm{H}$ & 1.202099 & -0.35415 & 1.669255 & 1.204245 & -0.34895 & 1.655338 \\
\hline $\mathrm{C}$ & -3.79538 & -3.53023 & -0.76017 & -3.79027 & -3.53173 & -0.73557 \\
\hline $\mathrm{O}$ & -3.59967 & -2.05948 & 1.15731 & -3.64102 & -2.02038 & 1.152536 \\
\hline $\mathrm{H}$ & -5.03688 & -1.18343 & -0.83065 & -5.06872 & -1.19561 & -0.67698 \\
\hline $\mathrm{H}$ & -4.3054 & 0.704402 & 0.28523 & -4.2974 & 0.705091 & 0.352085 \\
\hline $\mathrm{H}$ & -2.00955 & 2.576057 & 2.206969 & -1.99003 & 2.584833 & 2.178068 \\
\hline $\mathrm{H}$ & -1.67786 & 1.817363 & 3.77533 & -1.66648 & 1.818127 & 3.743523 \\
\hline $\mathrm{H}$ & 0.206898 & 3.303704 & 3.072795 & 0.221616 & 3.310765 & 3.052195 \\
\hline $\mathrm{H}$ & 0.708946 & 1.620016 & 3.280055 & 0.724269 & 1.62777 & 3.261721 \\
\hline $\mathrm{H}$ & -1.3734 & -3.18993 & 0.007583 & -1.31616 & -3.1458 & -0.15606 \\
\hline $\mathrm{H}$ & -1.40943 & -2.05412 & -1.33374 & -1.45823 & -1.93906 & -1.41793 \\
\hline $\mathrm{H}$ & -2.24628 & 1.977366 & -0.14485 & -2.2686 & 1.991209 & -0.17849 \\
\hline $\mathrm{H}$ & -1.65589 & 0.729456 & -1.23971 & -1.7139 & 0.738089 & -1.28745 \\
\hline $\mathrm{H}$ & 3.143613 & 1.973097 & -2.25136 & 3.185864 & 1.966443 & -2.25319 \\
\hline $\mathrm{H}$ & 3.53517 & 2.01565 & -0.52715 & 3.564287 & 2.011879 & -0.52625 \\
\hline $\mathrm{H}$ & 5.120822 & 0.288207 & -0.71464 & 5.145752 & 0.278683 & -0.69455 \\
\hline $\mathrm{H}$ & 5.963569 & -1.90624 & -1.54524 & 5.989318 & -1.91944 & -1.51467 \\
\hline $\mathrm{H}$ & 4.370197 & -2.28839 & -2.16613 & 4.400716 & -2.29724 & -2.15023 \\
\hline $\mathrm{H}$ & 5.360178 & -2.26124 & 0.704717 & 5.36422 & -2.27183 & 0.729519 \\
\hline $\mathrm{H}$ & 4.322258 & -3.42301 & -0.09971 & 4.328789 & -3.42975 & -0.08357 \\
\hline $\mathrm{H}$ & -4.46297 & -1.79089 & -3.03463 & 656 & -1.80429 & -2.92438 \\
\hline $\mathrm{H}$ & -2.80273 & -1.22645 & -2.90374 & -2.98558 & -1.25878 & -2.9101 \\
\hline $\mathrm{H}$ & -4.52919 & 0.683526 & -3.9179 & -4.74946 & 0.66007 & -3.82717 \\
\hline $\mathrm{H}$ & -4.92051 & 3.004933 & -2.80441 & -5.06107 & 2.991713 & -2.71583 \\
\hline $\mathrm{H}$ & -5.40375 & 2.851152 & -1.10377 & -5.4485 & 2.85392 & -0.98948 \\
\hline $\mathrm{H}$ & -3.74445 & 3.276911 & -1.50942 & -3.81333 & 3.270755 & -1.49149 \\
\hline $\mathrm{H}$ & 2.190371 & -3.45365 & 1.233736 & 2.180448 & -3.44784 & 1.232424 \\
\hline $\mathrm{H}$ & 3.449733 & -3.01544 & 2.400627 & 3.437589 & -3.02101 & 2.405832 \\
\hline $\mathrm{H}$ & 1.930655 & -2.11208 & 2.353993 & 1.927313 & -2.10447 & 2.353712 \\
\hline $\mathrm{H}$ & 5.875403 & 0.172853 & -2.86637 & 5.919273 & 0.161069 & -2.83883 \\
\hline $\mathrm{H}$ & 1.167786 & -0.37445 & -2.32937 & 1.206235 & -0.37149 & -2.34752 \\
\hline $\mathrm{H}$ & 2.606065 & -0.29589 & -3.37172 & 2.656447 & -0.30522 & -3.37346 \\
\hline $\mathrm{H}$ & 2.286006 & -1.71937 & -2.36348 & 2.316858 & -1.72325 & -2.364 \\
\hline $\mathrm{H}$ & -4.89287 & -3.54694 & -0.76709 & -4.88256 & -3.56683 & -0.67289 \\
\hline $\mathrm{H}$ & -3.44504 & -4.33495 & -0.10655 & -3.3889 & -4.3433 & -0.11646 \\
\hline $\mathrm{H}$ & -3.45268 & -3.73677 & -1.77995 & -3.49196 & -3.72552 & -1.77199 \\
\hline $\mathrm{O}$ & 0.264436 & -0.96327 & -0.08955 & -3.1131 & -2.64271 & 1.679409 \\
\hline
\end{tabular}




\begin{tabular}{|c|c|c|c|c|c|c|}
\hline & \multicolumn{3}{|c|}{$\mathrm{C3}$} & \multicolumn{3}{|c|}{$\mathrm{C} 4$} \\
\hline $\mathrm{O}$ & 0.264212 & -0.96151 & -0.088 & 0.281961 & -0.95427 & -0.11267 \\
\hline $\mathrm{C}$ & -1.65326 & 0.161169 & 0.808225 & -1.64109 & 0.181045 & 0.765939 \\
\hline $\mathrm{C}$ & -1.00109 & -1.18121 & 0.570601 & -0.99499 & -1.16098 & 0.514157 \\
\hline $\mathrm{C}$ & -1.47559 & 0.49479 & 2.097288 & -1.45776 & 0.505405 & 2.057925 \\
\hline $\mathrm{C}$ & -0.96524 & -0.70578 & 2.806274 & -0.97 & -0.70403 & 2.760763 \\
\hline $\mathrm{O}$ & -0.76258 & -1.70841 & 1.876835 & -0.79763 & -1.71618 & 1.823787 \\
\hline $\mathrm{O}$ & -0.72674 & -0.85756 & 3.978751 & -0.74132 & -0.87683 & 3.931796 \\
\hline $\mathrm{C}$ & -1.38276 & 1.844042 & 2.724117 & -1.36552 & 1.849813 & 2.694899 \\
\hline $\mathrm{C}$ & 0.120279 & 2.297948 & 2.655337 & 0.137407 & 2.304603 & 2.634694 \\
\hline $\mathrm{C}$ & 0.659049 & 2.32544 & 1.25534 & 0.682134 & 2.332014 & 1.237359 \\
\hline $\mathrm{C}$ & 1.023555 & 1.34359 & 0.405807 & 1.0437 & 1.348441 & 0.388892 \\
\hline $\mathrm{C}$ & 1.228451 & -0.13994 & 0.609774 & 1.241345 & -0.13498 & 0.595988 \\
\hline $\mathrm{C}$ & -1.73149 & -2.20117 & -0.30167 & -1.72762 & -2.15363 & -0.38159 \\
\hline $\mathrm{C}$ & -3.27268 & -2.17861 & -0.23954 & -3.2683 & -2.17471 & -0.22978 \\
\hline $\mathrm{C}$ & -3.96185 & -1.02769 & -1.02207 & -4.01787 & -1.03188 & -0.95738 \\
\hline $\mathrm{C}$ & -3.72612 & 0.462124 & -0.61618 & -3.7604 & 0.461963 & -0.57438 \\
\hline $\mathrm{C}$ & -2.2547 & 0.899797 & -0.33594 & -2.27788 & 0.912435 & -0.36194 \\
\hline $\mathrm{C}$ & 0.795189 & 3.620197 & 0.528838 & 0.829096 & 3.626337 & 0.511977 \\
\hline $\mathrm{O}$ & 1.279566 & 3.368152 & -0.7326 & 1.316717 & 3.371221 & -0.74755 \\
\hline $\mathrm{C}$ & 1.423721 & 1.951066 & -0.91007 & 1.455161 & 1.9536 & -0.92456 \\
\hline $\mathrm{C}$ & 2.855029 & 1.559926 & -1.28077 & 2.888008 & 1.556664 & -1.28391 \\
\hline $\mathrm{C}$ & 3.057407 & 0.019064 & -1.26784 & 3.086184 & 0.015373 & -1.26633 \\
\hline $\mathrm{C}$ & 2.633747 & -0.529 & 0.111684 & 2.649403 & -0.52962 & 0.110332 \\
\hline $\mathrm{C}$ & 4.57962 & -0.26444 & -1.47484 & 4.609603 & -0.27255 & -1.45922 \\
\hline $\mathrm{C}$ & 4.899284 & -1.75129 & -1.37055 & 4.924088 & -1.76029 & -1.35044 \\
\hline $\mathrm{C}$ & 4.509134 & -2.35455 & -0.01007 & 4.51946 & -2.36155 & 0.006772 \\
\hline $\mathrm{C}$ & 3.325776 & -1.70289 & 0.705989 & 3.3325 & -1.70492 & 0.711979 \\
\hline $\mathrm{C}$ & -3.83 & -1.02438 & -2.56866 & -3.98367 & -1.04135 & -2.50789 \\
\hline $\mathrm{C}$ & -4.25388 & 0.383581 & -2.91258 & -4.41436 & 0.367231 & -2.8381 \\
\hline $\mathrm{C}$ & -4.23913 & 1.199362 & -1.85061 & -4.33318 & 1.191654 & -1.78613 \\
\hline $\mathrm{O}$ & 0.54819 & 4.735035 & 0.921124 & 0.588033 & 4.742205 & 0.904349 \\
\hline $\mathrm{C}$ & -4.59438 & 2.655875 & -1.82148 & -4.68059 & 2.650028 & -1.74985 \\
\hline $\mathrm{C}$ & 2.677872 & -2.61822 & 1.727621 & 2.672705 & -2.61684 & 1.729234 \\
\hline $\mathrm{O}$ & 5.02425 & 0.164053 & -2.76438 & 5.066578 & 0.152872 & -2.74514 \\
\hline $\mathrm{C}$ & 2.241164 & -0.64392 & -2.40035 & 2.278515 & -0.64734 & -2.40519 \\
\hline $\mathrm{H}$ & 0.732151 & 1.660988 & -1.71073 & 0.768058 & 1.666552 & -1.73003 \\
\hline $\mathrm{O}$ & 3.613317 & -0.35194 & 1.159169 & 3.619809 & -0.35462 & 1.166519 \\
\hline $\mathrm{H}$ & 1.201398 & -0.36078 & 1.675022 & 1.203445 & -0.3559 & 1.661109 \\
\hline $\mathrm{C}$ & -3.79472 & -3.52915 & -0.76398 & -3.78989 & -3.53048 & -0.74007 \\
\hline $\mathrm{O}$ & -3.60061 & -2.05841 & 1.153932 & -3.64256 & -2.01927 & 1.148608 \\
\hline $\mathrm{H}$ & -5.03657 & -1.18253 & -0.83504 & -5.06817 & -1.19468 & -0.68292 \\
\hline
\end{tabular}




\begin{tabular}{|c|c|c|c|c|c|c|}
\hline $\mathrm{H}$ & -4.30662 & 0.70466 & 0.282789 & -4.29875 & 0.705171 & 0.349345 \\
\hline $\mathrm{H}$ & -2.01132 & 2.576371 & 2.208141 & -1.99212 & 2.584453 & 2.18013 \\
\hline $\mathrm{H}$ & -1.68223 & 1.816229 & 3.77628 & -1.67077 & 1.816124 & 3.745172 \\
\hline $\mathrm{H}$ & 0.203477 & 3.304037 & 3.078253 & 0.217873 & 3.310625 & 3.058219 \\
\hline $\mathrm{H}$ & 0.705363 & 1.620389 & 3.286394 & 0.720659 & 1.627734 & 3.26839 \\
\hline $\mathrm{H}$ & -1.37323 & -3.18867 & 0.004852 & -1.3163 & -3.14501 & -0.15742 \\
\hline $\mathrm{H}$ & -1.40824 & -2.05134 & -1.33517 & -1.45625 & -1.93764 & -1.41884 \\
\hline $\mathrm{H}$ & -2.24735 & 1.978357 & -0.14502 & -2.26943 & 1.992077 & -0.17771 \\
\hline $\mathrm{H}$ & -1.65594 & 0.730246 & -1.23965 & -1.71333 & 0.739217 & -1.28676 \\
\hline $\mathrm{H}$ & 3.098804 & 1.964348 & -2.26974 & 3.140462 & 1.95815 & -2.27183 \\
\hline $\mathrm{H}$ & 3.527891 & 2.024401 & -0.54829 & 3.556569 & 2.020977 & -0.54739 \\
\hline $\mathrm{H}$ & 5.128054 & 0.275938 & -0.69269 & 5.152496 & 0.267314 & -0.67286 \\
\hline $\mathrm{H}$ & 5.970635 & -1.88782 & -1.55102 & 5.99668 & -1.89997 & -1.52055 \\
\hline $\mathrm{H}$ & 4.385038 & -2.2741 & -2.18411 & 4.416133 & -2.28229 & -2.16843 \\
\hline $\mathrm{H}$ & 5.352312 & -2.28601 & 0.690002 & 5.356554 & -2.29622 & 0.714394 \\
\hline $\mathrm{H}$ & 4.302303 & -3.42453 & -0.13047 & 4.309332 & -3.43073 & -0.11509 \\
\hline $\mathrm{H}$ & -4.46058 & -1.78829 & -3.03893 & -4.652 & -1.80129 & -2.93013 \\
\hline $\mathrm{H}$ & -2.80048 & -1.22418 & -2.90609 & -2.98117 & -1.25561 & -2.91253 \\
\hline $\mathrm{H}$ & -4.52618 & 0.686498 & -3.92064 & -4.74419 & 0.663681 & -3.83079 \\
\hline $\mathrm{H}$ & -4.91908 & 3.007127 & -2.80608 & -5.05845 & 2.994281 & -2.71786 \\
\hline $\mathrm{H}$ & -5.40535 & 2.851408 & -1.10656 & -5.44996 & 2.854064 & -0.99268 \\
\hline $\mathrm{H}$ & -3.74564 & 3.278811 & -1.50861 & -3.81393 & 3.272892 & -1.49009 \\
\hline $\mathrm{H}$ & 2.182678 & -3.4554 & 1.22346 & 1.935659 & -2.11218 & 2.35389 \\
\hline $\mathrm{H}$ & 3.452792 & -3.03217 & 2.384042 & 2.173483 & -3.44968 & 1.221796 \\
\hline $\mathrm{H}$ & 1.93891 & -2.11948 & 2.354501 & 3.441065 & -3.03748 & 2.389026 \\
\hline $\mathrm{H}$ & 5.160715 & 1.122391 & -2.73255 & 5.208815 & 1.110355 & -2.71294 \\
\hline $\mathrm{H}$ & 1.189306 & -0.35332 & -2.37808 & 1.228044 & -0.35106 & -2.39564 \\
\hline $\mathrm{H}$ & 2.668859 & -0.35939 & -3.36618 & 2.718087 & -0.36792 & -3.36712 \\
\hline $\mathrm{H}$ & 2.262544 & -1.73464 & -2.32273 & 2.293438 & -1.73793 & -2.32413 \\
\hline $\mathrm{H}$ & -4.89219 & -3.54612 & -0.77165 & -4.88225 & -3.56554 & -0.67862 \\
\hline $\mathrm{H}$ & -3.44457 & -4.33399 & -0.11041 & -3.38923 & -4.34237 & -0.12094 \\
\hline $\mathrm{H}$ & -3.4513 & -3.73534 & -1.78358 & -3.4904 & -3.72391 & -1.7762 \\
\hline $\mathrm{H}$ & -4.56768 & -2.09385 & 1.233264 & -3.12003 & -2.64623 & 1.675396 \\
\hline
\end{tabular}

Table S8. Cartesian coordinates for 2.

\begin{tabular}{|l|l|l|l|l|l|l|}
\hline & \multicolumn{3}{|c}{ C1 } & \multicolumn{3}{c|}{ C2 } \\
\hline H & -0.03281 & 0.356469 & -1.7821 & -0.04308 & 0.355448 & -1.78229 \\
\hline H & -1.49081 & 1.309518 & 1.740348 & -1.50189 & 1.320185 & 1.732098 \\
\hline H & 2.591794 & 2.80956 & -1.54426 & 2.591725 & 2.804646 & -1.54382 \\
\hline C & -1.54888 & 2.446026 & -0.06658 & -1.55029 & 2.449634 & -0.07905 \\
\hline O & 5.140878 & -0.04434 & 0.908475 & 5.170243 & -0.17717 & 0.925136 \\
\hline
\end{tabular}




\begin{tabular}{|c|c|c|c|c|c|c|}
\hline $\mathrm{C}$ & 0.83467 & -2.24297 & -2.21181 & 0.823323 & -2.25101 & -2.21001 \\
\hline $\mathrm{C}$ & 4.08686 & 0.617235 & -1.74653 & 4.092334 & 0.608991 & -1.7489 \\
\hline $\mathrm{O}$ & 0.052557 & 5.094657 & 0.639098 & 0.061569 & 5.090969 & 0.644498 \\
\hline $\mathrm{C}$ & -0.13212 & 2.838061 & -0.25108 & -0.13156 & 2.838822 & -0.25644 \\
\hline $\mathrm{C}$ & 0.53263 & 4.085203 & 0.182032 & 0.536893 & 4.081181 & 0.184385 \\
\hline $\mathrm{O}$ & 1.889554 & 3.906675 & 0.017961 & 1.895193 & 3.896773 & 0.024867 \\
\hline $\mathrm{C}$ & 0.550729 & 0.510476 & -0.8649 & 0.544503 & 0.508293 & -0.86747 \\
\hline $\mathrm{C}$ & 0.796872 & 1.974769 & -0.66515 & 0.795353 & 1.971912 & -0.6679 \\
\hline $\mathrm{C}$ & 2.14226 & 2.613133 & -0.5648 & 2.142503 & 2.606363 & -0.56483 \\
\hline $\mathrm{C}$ & 3.039866 & 1.703904 & 0.265154 & 3.040796 & 1.689975 & 0.256816 \\
\hline $\mathrm{C}$ & 1.921908 & -1.5488 & -1.42844 & 1.917125 & -1.55011 & -1.44259 \\
\hline $\mathrm{C}$ & 1.865712 & -0.29207 & -0.93448 & 1.858943 & -0.29475 & -0.945 \\
\hline $\mathrm{C}$ & 3.209104 & 0.35728 & -0.48739 & 3.204195 & 0.345612 & -0.49975 \\
\hline $\mathrm{C}$ & 3.930819 & -0.65129 & 0.437115 & 3.926163 & -0.67805 & 0.424823 \\
\hline $\mathrm{C}$ & 4.25601 & -1.95399 & -0.30849 & 4.260316 & -1.96102 & -0.3363 \\
\hline $\mathrm{C}$ & 3.145906 & -2.43665 & -1.27283 & 3.155417 & -2.42351 & -1.31605 \\
\hline $\mathrm{O}$ & -0.58436 & -4.30275 & 0.324869 & -0.59959 & -4.29134 & 0.381187 \\
\hline $\mathrm{C}$ & -0.33115 & -3.55998 & 1.517032 & -0.30918 & -3.53948 & 1.558533 \\
\hline $\mathrm{H}$ & -2.4463 & 0.11429 & -1.51527 & -2.4377 & 0.092752 & -1.5187 \\
\hline $\mathrm{C}$ & -3.3614 & -0.39592 & 1.800838 & -3.37223 & -0.37824 & 1.797223 \\
\hline $\mathrm{H}$ & -5.43542 & 1.278988 & -1.79672 & -5.42592 & 1.250816 & -1.83237 \\
\hline $\mathrm{H}$ & -4.05701 & 2.410168 & 0.152881 & -4.05996 & 2.408302 & 0.110395 \\
\hline $\mathrm{C}$ & -4.92941 & -1.6693 & -1.89596 & -4.91717 & -1.69839 & -1.88971 \\
\hline $\mathrm{O}$ & 1.419812 & -1.48971 & 2.850164 & 1.418824 & -1.44063 & 2.875866 \\
\hline $\mathrm{O}$ & 0.457521 & 0.068518 & 1.515545 & 0.455643 & 0.094009 & 1.516586 \\
\hline $\mathrm{C}$ & 0.653505 & -1.21514 & 1.953434 & 0.654861 & -1.1835 & 1.972344 \\
\hline $\mathrm{C}$ & -0.23296 & -2.10534 & 1.181826 & -0.22454 & -2.0874 & 1.208831 \\
\hline $\mathrm{C}$ & -1.63466 & 1.083676 & 0.679459 & -1.64034 & 1.089816 & 0.671475 \\
\hline $\mathrm{C}$ & -0.42727 & 0.073555 & 0.364913 & -0.43031 & 0.080633 & 0.3671 \\
\hline $\mathrm{C}$ & -0.91086 & -1.36733 & 0.286348 & -0.9075 & -1.36316 & 0.305772 \\
\hline $\mathrm{C}$ & -2.10531 & -1.75772 & -0.54432 & -2.10103 & -1.76645 & -0.52082 \\
\hline $\mathrm{C}$ & -4.21837 & 1.338912 & 0.147137 & -4.22063 & 1.336921 & 0.117758 \\
\hline $\mathrm{C}$ & -3.0351 & 0.39257 & 0.509914 & -3.03905 & 0.396043 & 0.499521 \\
\hline $\mathrm{C}$ & -2.95861 & -0.51049 & -0.76261 & -2.95367 & -0.52284 & -0.7609 \\
\hline $\mathrm{C}$ & -4.37799 & -0.62513 & -1.27523 & -4.37012 & -0.64553 & -1.27994 \\
\hline $\mathrm{C}$ & -5.02993 & 0.697385 & -0.97191 & -5.02501 & 0.680208 & -0.9977 \\
\hline $\mathrm{C}$ & -5.63401 & 0.880838 & 0.401466 & -5.63754 & 0.881264 & 0.369449 \\
\hline $\mathrm{H}$ & -2.08138 & 3.213077 & 0.502821 & -2.0863 & 3.218917 & 0.483938 \\
\hline $\mathrm{H}$ & -2.04604 & 2.373315 & -1.04372 & -2.04053 & 2.372914 & -1.05929 \\
\hline $\mathrm{H}$ & 5.432587 & -0.54199 & 1.686887 & 4.977394 & 0.36998 & 1.701088 \\
\hline $\mathrm{H}$ & 1.27623 & -2.6693 & -3.12362 & 1.254524 & -2.68347 & -3.12374 \\
\hline $\mathrm{H}$ & 0.394511 & -3.07699 & -1.65324 & 0.391602 & -3.08168 & -1.63965 \\
\hline $\mathrm{H}$ & 0.032871 & -1.57266 & -2.52934 & 0.016044 & -1.5846 & -2.52216 \\
\hline
\end{tabular}




\begin{tabular}{|l|l|l|l|l|l|l|}
\hline $\mathrm{H}$ & 5.056032 & 1.030405 & -1.44871 & 5.076809 & 0.968932 & -1.43194 \\
\hline $\mathrm{H}$ & 4.267452 & -0.29864 & -2.31353 & 4.237724 & -0.29472 & -2.34433 \\
\hline $\mathrm{H}$ & 3.611887 & 1.318435 & -2.4388 & 3.653144 & 1.352374 & -2.42042 \\
\hline $\mathrm{H}$ & 4.021442 & 2.155216 & 0.424662 & 4.024103 & 2.147529 & 0.402805 \\
\hline $\mathrm{H}$ & 2.57765 & 1.544966 & 1.244887 & 2.579106 & 1.534883 & 1.238769 \\
\hline $\mathrm{H}$ & 3.272386 & -0.85169 & 1.290075 & 3.260501 & -0.89994 & 1.266678 \\
\hline $\mathrm{H}$ & 4.482108 & -2.73867 & 0.424094 & 4.483634 & -2.74998 & 0.389042 \\
\hline $\mathrm{H}$ & 5.183987 & -1.77792 & -0.86113 & 5.19375 & -1.77753 & -0.87804 \\
\hline $\mathrm{H}$ & 3.572846 & -2.63416 & -2.26757 & 3.581525 & -2.5801 & -2.31862 \\
\hline $\mathrm{H}$ & 2.76707 & -3.41378 & -0.94088 & 2.794877 & -3.41786 & -1.0171 \\
\hline $\mathrm{H}$ & -0.65984 & -5.23767 & 0.569916 & -0.65321 & -5.22583 & 0.633379 \\
\hline $\mathrm{H}$ & -1.13662 & -3.71986 & 2.254401 & -1.08911 & -3.69469 & 2.32386 \\
\hline $\mathrm{H}$ & 0.608747 & -3.86399 & 1.999479 & 0.646548 & -3.838 & 2.012658 \\
\hline $\mathrm{H}$ & -2.49676 & -0.94282 & 2.182663 & -2.50962 & -0.92189 & 2.188431 \\
\hline $\mathrm{H}$ & -3.68307 & 0.299422 & 2.583835 & -3.69686 & 0.325507 & 2.571463 \\
\hline $\mathrm{H}$ & -4.16552 & -1.12267 & 1.648624 & -4.17607 & -1.10603 & 1.648937 \\
\hline $\mathrm{H}$ & -5.95929 & -1.6465 & -2.24253 & -5.94515 & -1.68119 & -2.24216 \\
\hline $\mathrm{H}$ & -4.3726 & -2.58279 & -2.08943 & -4.35856 & -2.61389 & -2.06775 \\
\hline $\mathrm{H}$ & -2.66358 & -2.53983 & -0.01896 & -2.66047 & -2.53956 & 0.01611 \\
\hline $\mathrm{H}$ & -1.80654 & -2.199 & -1.50038 & -1.80099 & -2.22356 & -1.46881 \\
\hline $\mathrm{H}$ & -6.38256 & 1.663105 & 0.502787 & -6.38718 & 1.664274 & 0.455935 \\
\hline $\mathrm{H}$ & -5.83417 & 0.004295 & 1.008008 & -5.84079 & 0.012706 & 0.986346 \\
\hline
\end{tabular}

\begin{tabular}{|l|l|l|l|}
\hline \multicolumn{5}{|c|}{ C3 } \\
\hline H & 0.112471 & 0.431827 & 1.80973 \\
\hline H & 1.595387 & 1.108514 & -1.77958 \\
\hline H & -2.33462 & 3.041374 & 1.462209 \\
\hline C & 1.754044 & 2.330677 & -0.0355 \\
\hline O & -5.13139 & 0.291338 & -0.77759 \\
\hline C & -0.91544 & -2.10623 & 2.320851 \\
\hline C & -3.93383 & 0.955802 & 1.806467 \\
\hline O & 0.324759 & 5.035796 & -0.85704 \\
\hline C & 0.367629 & 2.819658 & 0.144313 \\
\hline C & -0.21747 & 4.086233 & -0.34479 \\
\hline O & -1.58033 & 4.008107 & -0.15963 \\
\hline C & -0.46064 & 0.576269 & 0.884433 \\
\hline C & -0.61136 & 2.042029 & 0.610232 \\
\hline C & -1.9119 & 2.765423 & 0.490317 \\
\hline C & -2.87977 & 1.880991 & -0.28473 \\
\hline C & -1.95073 & -1.38921 & 1.489366 \\
\hline C & -1.82237 & -0.1442 & 0.976524 \\
\hline C & -3.12554 & 0.582668 & 0.528847 \\
\hline C & -3.95659 & -0.39752 & -0.33329 \\
\hline
\end{tabular}




\begin{tabular}{|c|c|c|c|}
\hline $\mathrm{C}$ & -4.34689 & -1.64308 & 0.468377 \\
\hline $\mathrm{C}$ & -3.18062 & -2.25545 & 1.27037 \\
\hline $\mathrm{O}$ & -1.25858 & -4.15254 & -1.23636 \\
\hline $\mathrm{C}$ & 0.086385 & -3.68934 & -1.21735 \\
\hline $\mathrm{H}$ & 2.543338 & 0.052744 & 1.525397 \\
\hline $\mathrm{C}$ & 3.352716 & -0.72114 & -1.76849 \\
\hline $\mathrm{H}$ & 5.602797 & 1.050458 & 1.675682 \\
\hline $\mathrm{H}$ & 4.251129 & 2.13447 & -0.31887 \\
\hline $\mathrm{C}$ & 4.924822 & -1.849 & 1.979235 \\
\hline $\mathrm{O}$ & -1.51505 & -1.61677 & -2.63754 \\
\hline $\mathrm{O}$ & -0.41344 & -0.02027 & -1.464 \\
\hline $\mathrm{C}$ & -0.7007 & -1.31332 & -1.7852 \\
\hline $\mathrm{C}$ & 0.143324 & -2.20524 & -0.9734 \\
\hline $\mathrm{C}$ & 1.743968 & 0.92958 & -0.71035 \\
\hline $\mathrm{C}$ & 0.488547 & 0.012062 & -0.32184 \\
\hline $\mathrm{C}$ & 0.885378 & -1.44763 & -0.14965 \\
\hline $\mathrm{C}$ & 2.071046 & -1.85305 & 0.688756 \\
\hline $\mathrm{C}$ & 4.34864 & 1.05805 & -0.24537 \\
\hline $\mathrm{C}$ & 3.103392 & 0.164481 & -0.52437 \\
\hline $\mathrm{C}$ & 3.002949 & -0.64803 & 0.806743 \\
\hline $\mathrm{C}$ & 4.423524 & -0.81568 & 1.300612 \\
\hline $\mathrm{C}$ & 5.145416 & 0.442301 & 0.898644 \\
\hline $\mathrm{C}$ & 5.728156 & 0.498726 & -0.49487 \\
\hline $\mathrm{H}$ & 2.322927 & 3.033247 & -0.6509 \\
\hline $\mathrm{H}$ & 2.261634 & 2.278089 & 0.937593 \\
\hline $\mathrm{H}$ & -5.55377 & -0.2601 & -1.45354 \\
\hline $\mathrm{H}$ & -1.38545 & -2.42869 & 3.260385 \\
\hline $\mathrm{H}$ & -0.54758 & -3.01696 & 1.833093 \\
\hline $\mathrm{H}$ & -0.0604 & -1.48317 & 2.589058 \\
\hline $\mathrm{H}$ & -4.87991 & 1.431526 & 1.528465 \\
\hline $\mathrm{H}$ & -4.15679 & 0.074572 & 2.411963 \\
\hline $\mathrm{H}$ & -3.38048 & 1.640407 & 2.455834 \\
\hline $\mathrm{H}$ & -3.83206 & 2.388698 & -0.45174 \\
\hline $\mathrm{H}$ & -2.44533 & 1.648312 & -1.26276 \\
\hline $\mathrm{H}$ & -3.34782 & -0.67746 & -1.20241 \\
\hline $\mathrm{H}$ & -4.76263 & -2.39734 & -0.2123 \\
\hline $\mathrm{H}$ & -5.16432 & -1.35524 & 1.136552 \\
\hline $\mathrm{H}$ & -3.54598 & -2.63441 & 2.235585 \\
\hline $\mathrm{H}$ & -2.8013 & -3.14153 & 0.739959 \\
\hline $\mathrm{H}$ & -1.70777 & -3.62271 & -1.92177 \\
\hline $\mathrm{H}$ & 0.598698 & -4.23757 & -0.42175 \\
\hline $\mathrm{H}$ & 0.596774 & -3.92951 & -2.16492 \\
\hline $\mathrm{H}$ & 2.448906 & -1.23552 & -2.10121 \\
\hline
\end{tabular}




\begin{tabular}{|l|l|l|l|}
\hline $\mathrm{H}$ & 3.699904 & -0.09629 & -2.59846 \\
\hline $\mathrm{H}$ & 4.114439 & -1.48562 & -1.58596 \\
\hline $\mathrm{H}$ & 5.96155 & -1.8669 & 2.304613 \\
\hline $\mathrm{H}$ & 4.319541 & -2.71244 & 2.244142 \\
\hline $\mathrm{H}$ & 2.582583 & -2.6953 & 0.208597 \\
\hline $\mathrm{H}$ & 1.768482 & -2.20317 & 1.68223 \\
\hline $\mathrm{H}$ & 6.519553 & 1.225531 & -0.66158 \\
\hline $\mathrm{H}$ & 5.861857 & -0.42608 & -1.0454 \\
\hline
\end{tabular}

Table S9. Frequencies data for $1 \mathrm{a}$.

\begin{tabular}{|c|c|c|c|c|c|}
\hline \multicolumn{3}{|c|}{$\mathrm{C1}$} & \multicolumn{3}{|c|}{$\mathrm{C2}$} \\
\hline 1 & 2 & 3 & 1 & 2 & 3 \\
\hline 29.0483 & 32.4005 & 46.9257 & 29.4813 & 32.6330 & 46.5082 \\
\hline 4 & 5 & 6 & 4 & 5 & 6 \\
\hline 55.7137 & 66.7711 & 77.3714 & 54.1213 & 65.9101 & 77.7873 \\
\hline 7 & 8 & 9 & 7 & 8 & 9 \\
\hline 94.0323 & 94.7874 & 114.7449 & 94.0320 & 95.6827 & 115.7000 \\
\hline 10 & 11 & 12 & 10 & 11 & 12 \\
\hline 127.1909 & 141.8787 & 154.0392 & 127.1553 & 143.2496 & 153.8956 \\
\hline 13 & 14 & 15 & 13 & 14 & 15 \\
\hline 154.4952 & 163.7665 & 169.9526 & 154.9235 & 163.5721 & 170.3102 \\
\hline 16 & 17 & 18 & 16 & 17 & 18 \\
\hline 175.8722 & 178.8438 & 194.5557 & 174.2440 & 179.0832 & 193.4316 \\
\hline 19 & 20 & 21 & 19 & 20 & 21 \\
\hline 206.2073 & 212.8883 & 224.1884 & 205.7240 & 215.8273 & 225.3697 \\
\hline 22 & 23 & 24 & 22 & 23 & 24 \\
\hline 240.4204 & 247.8885 & 251.4109 & 241.8181 & 246.1129 & 251.5124 \\
\hline 25 & 26 & 27 & 25 & 26 & 27 \\
\hline 259.7702 & 267.3237 & 277.6180 & 261.0730 & 267.5354 & 274.3566 \\
\hline 28 & 29 & 30 & 28 & 29 & 30 \\
\hline 284.4923 & 290.4393 & 297.6173 & 283.5408 & 291.5849 & 297.6866 \\
\hline 31 & 32 & 33 & 31 & 32 & 33 \\
\hline 312.1457 & 316.5659 & 319.7747 & 297.9948 & 312.3929 & 316.7533 \\
\hline 34 & 35 & 36 & 34 & 35 & 36 \\
\hline 337.4579 & 344.7992 & 348.2635 & 321.3474 & 343.1636 & 346.3674 \\
\hline 37 & 38 & 39 & 37 & 38 & 39 \\
\hline 360.4746 & 364.8636 & 369.6937 & 362.3827 & 365.2262 & 369.5614 \\
\hline 40 & 41 & 42 & 40 & 41 & 42 \\
\hline 373.2237 & 380.0267 & 413.2829 & 373.6931 & 380.3535 & 413.1261 \\
\hline 43 & 44 & 45 & 43 & 44 & 45 \\
\hline 436.0060 & 446.8259 & 462.7128 & 435.3886 & 446.8271 & 460.8370 \\
\hline 46 & 47 & 48 & 46 & 47 & 48 \\
\hline 467.4816 & 483.2972 & 496.8014 & 465.7140 & 480.4352 & 495.7037 \\
\hline
\end{tabular}




\begin{tabular}{|c|c|c|c|c|c|}
\hline 49 & 50 & 51 & 49 & 50 & 51 \\
\hline 512.1446 & 518.2600 & 527.3690 & 511.5490 & 516.1867 & 528.0172 \\
\hline 52 & 53 & 54 & 52 & 53 & 54 \\
\hline 541.8275 & 546.6963 & 557.6614 & 541.9106 & 547.7804 & 558.5947 \\
\hline 55 & 56 & 57 & 55 & 56 & 57 \\
\hline 581.3383 & 589.5202 & 593.9436 & 581.6343 & 589.8264 & 595.1055 \\
\hline 58 & 59 & 60 & 58 & 59 & 60 \\
\hline 612.5161 & 620.4675 & 631.9908 & 614.2622 & 620.2542 & 631.2951 \\
\hline 61 & 62 & 63 & 61 & 62 & 63 \\
\hline 637.6768 & 658.9198 & 659.6447 & 638.0305 & 658.5369 & 659.8450 \\
\hline 64 & 65 & 66 & 64 & 65 & 66 \\
\hline 676.2683 & 686.0763 & 700.1676 & 677.9263 & 686.2031 & 702.9731 \\
\hline 67 & 68 & 69 & 67 & 68 & 69 \\
\hline 735.3853 & 744.2646 & 750.9794 & 736.0998 & 744.6811 & 751.2703 \\
\hline 70 & 71 & 72 & 70 & 71 & 72 \\
\hline 765.5673 & 771.4452 & 777.6317 & 765.8952 & 772.4575 & 778.7815 \\
\hline 73 & 74 & 75 & 73 & 74 & 75 \\
\hline 789.2908 & 792.9024 & 825.6421 & 789.2476 & 793.4420 & 825.5387 \\
\hline 76 & 77 & 78 & 76 & 77 & 78 \\
\hline 834.0098 & 840.0923 & 857.2985 & 833.5694 & 840.4647 & 857.6387 \\
\hline 79 & 80 & 81 & 79 & 80 & 81 \\
\hline 866.4042 & 876.6986 & 884.1222 & 868.0403 & 876.6684 & 884.5558 \\
\hline 82 & 83 & 84 & 82 & 83 & 84 \\
\hline 893.9236 & 907.4810 & 920.7115 & 894.1801 & 912.0003 & 921.6080 \\
\hline 85 & 86 & 87 & 85 & 86 & 87 \\
\hline 930.4030 & 937.3947 & 946.9718 & 930.3871 & 939.2087 & 948.7539 \\
\hline 88 & 89 & 90 & 88 & 89 & 90 \\
\hline 952.5422 & 956.8249 & 961.3770 & 955.1225 & 957.1806 & 961.1233 \\
\hline 91 & 92 & 93 & 91 & 92 & 93 \\
\hline 976.5403 & 981.3396 & 984.2139 & 976.8201 & 984.4913 & 985.6213 \\
\hline 94 & 95 & 96 & 94 & 95 & 96 \\
\hline 999.2833 & 1004.2277 & 1012.8212 & 998.6789 & 1003.9273 & 1011.7256 \\
\hline 97 & 98 & 99 & 97 & 98 & 99 \\
\hline 1013.9116 & 1020.5656 & 1035.0901 & 1013.6266 & 1020.2654 & 1035.0623 \\
\hline 100 & 101 & 102 & 100 & 101 & 102 \\
\hline 1041.9696 & 1046.0796 & 1053.8623 & 1042.2989 & 1047.4595 & 1054.1712 \\
\hline 103 & 104 & 105 & 103 & 104 & 105 \\
\hline 1059.3274 & 1065.2374 & 1080.1530 & 1057.8969 & 1065.3084 & 1080.2013 \\
\hline 106 & 107 & 108 & 106 & 107 & 108 \\
\hline 1088.1900 & 1100.8358 & 1104.0858 & 1086.9036 & 1100.1114 & 1103.0532 \\
\hline 109 & 110 & 111 & 109 & 110 & 111 \\
\hline 1107.6405 & 1109.9022 & 1125.3881 & 1109.9110 & 1110.3648 & 1125.8282 \\
\hline 112 & 113 & 114 & 112 & 113 & 114 \\
\hline
\end{tabular}




\begin{tabular}{|c|c|c|c|c|c|}
\hline 1130.1269 & 1152.7241 & 1156.4695 & 1132.9056 & 1152.4227 & 1155.9297 \\
\hline 115 & 116 & 117 & 115 & 116 & 117 \\
\hline 1159.8954 & 1170.6470 & 1172.5529 & 1160.2749 & 1170.4031 & 1173.0392 \\
\hline 118 & 119 & 120 & 118 & 119 & 120 \\
\hline 1182.4237 & 1186.1712 & 1194.3181 & 1179.9348 & 1186.3478 & 1194.3465 \\
\hline 121 & 122 & 123 & 121 & 122 & 123 \\
\hline 1197.5657 & 1213.2624 & 1216.1951 & 1203.8380 & 1215.1421 & 1216.4832 \\
\hline 124 & 125 & 126 & 124 & 125 & 126 \\
\hline 1227.3873 & 1233.6531 & 1234.9657 & 1229.3446 & 1234.0094 & 1236.0934 \\
\hline 127 & 128 & 129 & 127 & 128 & 129 \\
\hline 1244.4907 & 1259.4609 & 1263.5154 & 1245.4144 & 1263.4165 & 1273.1715 \\
\hline 130 & 131 & 132 & 130 & 131 & 132 \\
\hline 1278.9301 & 1297.7503 & 1314.7716 & 1282.4582 & 1297.9221 & 1309.0694 \\
\hline 133 & 134 & 135 & 133 & 134 & 135 \\
\hline 1318.1112 & 1320.4833 & 1323.4500 & 1318.4018 & 1320.4824 & 1323.5627 \\
\hline 136 & 137 & 138 & 136 & 137 & 138 \\
\hline 1329.9376 & 1335.8395 & 1338.4901 & 1329.4361 & 1334.9195 & 1338.6382 \\
\hline 139 & 140 & 141 & 139 & 140 & 141 \\
\hline 1341.8716 & 1348.7653 & 1352.2524 & 1340.2746 & 1348.6910 & 1352.2056 \\
\hline 142 & 143 & 144 & 142 & 143 & 144 \\
\hline 1360.9837 & 1369.1690 & 1374.8819 & 1360.5953 & 1364.6898 & 1372.1451 \\
\hline 145 & 146 & 147 & 145 & 146 & 147 \\
\hline 1382.1127 & 1385.4416 & 1388.4877 & 1378.6099 & 1381.5385 & 1388.3659 \\
\hline 148 & 149 & 150 & 148 & 149 & 150 \\
\hline 1392.7339 & 1401.2917 & 1404.2931 & 1389.0240 & 1393.5254 & 1404.4055 \\
\hline 151 & 152 & 153 & 151 & 152 & 153 \\
\hline 1406.6214 & 1412.4772 & 1415.6584 & 1406.8839 & 1412.5417 & 1431.0909 \\
\hline 154 & 155 & 156 & 154 & 155 & 156 \\
\hline 1436.1978 & 1436.7355 & 1444.6370 & 1436.2369 & 1438.3155 & 1444.6087 \\
\hline 157 & 158 & 159 & 157 & 158 & 159 \\
\hline 1445.8998 & 1455.7519 & 1470.5978 & 1446.0115 & 1455.9333 & 1471.0307 \\
\hline 160 & 161 & 162 & 160 & 161 & 162 \\
\hline 1502.4737 & 1508.0947 & 1509.3863 & 1497.9449 & 1507.1871 & 1509.3785 \\
\hline 163 & 164 & 165 & 163 & 164 & 165 \\
\hline 1510.0279 & 1514.7951 & 1516.2589 & 1509.8235 & 1514.8615 & 1516.2285 \\
\hline 166 & 167 & 168 & 166 & 167 & 168 \\
\hline 1517.9060 & 1519.5592 & 1521.0546 & 1517.6441 & 1519.4200 & 1520.8227 \\
\hline 169 & 170 & 171 & 169 & 170 & 171 \\
\hline 1522.9930 & 1523.9787 & 1525.6152 & 1523.0238 & 1523.1069 & 1525.6253 \\
\hline 172 & 173 & 174 & 172 & 173 & 174 \\
\hline 1526.1250 & 1529.2192 & 1534.0314 & 1527.2659 & 1528.8339 & 1534.4364 \\
\hline 175 & 176 & 177 & 175 & 176 & 177 \\
\hline 1542.7276 & 1703.8983 & 1728.6124 & 1543.0541 & 1702.9710 & 1730.1401 \\
\hline
\end{tabular}




\begin{tabular}{|c|c|c|c|c|c|}
\hline 178 & 179 & 180 & 178 & 179 & 180 \\
\hline 1731.5683 & 1854.8349 & 1857.8374 & 1733.7511 & 1853.9721 & 1856.9585 \\
\hline 181 & 182 & 183 & 181 & 182 & 183 \\
\hline 3003.0854 & 3031.5742 & 3041.3599 & 2988.5464 & 3011.4885 & 3031.6677 \\
\hline 184 & 185 & 186 & 184 & 185 & 186 \\
\hline 3047.6814 & 3049.6480 & 3050.3173 & 3044.4234 & 3047.3688 & 3049.2716 \\
\hline 187 & 188 & 189 & 187 & 188 & 189 \\
\hline 3050.5906 & 3055.0344 & 3056.5945 & 3049.9640 & 3054.7610 & 3055.3971 \\
\hline 190 & 191 & 192 & 190 & 191 & 192 \\
\hline 3060.5586 & 3064.6107 & 3066.1568 & 3056.1105 & 3059.9111 & 3065.4008 \\
\hline 193 & 194 & 195 & 193 & 194 & 195 \\
\hline 3070.5395 & 3070.9039 & 3071.4152 & 3069.9202 & 3071.8844 & 3075.4058 \\
\hline 196 & 197 & 198 & 196 & 197 & 198 \\
\hline 3075.3655 & 3076.7594 & 3080.5419 & 3076.8932 & 3079.1523 & 3089.5808 \\
\hline 199 & 200 & 201 & 199 & 200 & 201 \\
\hline 3089.9067 & 3099.6802 & 3103.3115 & 3089.6968 & 3098.4969 & 3102.8271 \\
\hline 202 & 203 & 204 & 202 & 203 & 204 \\
\hline 3109.9416 & 3112.2156 & 3112.3142 & 3109.4965 & 3111.5773 & 3111.7817 \\
\hline 205 & 206 & 207 & 205 & 206 & 207 \\
\hline 3120.1254 & 3122.2111 & 3122.5252 & 3120.0063 & 3122.7706 & 3129.4602 \\
\hline 208 & 209 & 210 & 208 & 209 & 210 \\
\hline 3130.5519 & 3132.1372 & 3149.9879 & 3132.6951 & 3138.1884 & 3149.7697 \\
\hline 211 & 212 & 213 & 211 & 212 & 213 \\
\hline 3156.4895 & 3177.9784 & 3180.9716 & 3157.4147 & 3177.8884 & 3184.1130 \\
\hline 214 & 215 & 216 & 214 & 215 & 216 \\
\hline 3194.4658 & 3656.9995 & 3726.4801 & 3194.8126 & 3655.0022 & 3726.3743 \\
\hline
\end{tabular}

Table S10. Frequencies data for $1 \mathrm{~b}$.

\begin{tabular}{|c|c|c|c|c|c|}
\hline \multicolumn{7}{|c|}{ C1 } & \multicolumn{3}{c|}{ C2 } \\
\hline 1 & 2 & 3 & 1 & 2 & 3 \\
\hline 29.2524 & 33.6128 & 43.3023 & 29.7056 & 33.0467 & 43.5842 \\
\hline 4 & 5 & 6 & 4 & 5 & 6 \\
\hline 53.9076 & 68.5131 & 77.8995 & 55.7504 & 68.7421 & 77.9232 \\
\hline 7 & 8 & 9 & 7 & 8 & 9 \\
\hline 92.7419 & 96.0602 & 110.1344 & 92.3297 & 95.8396 & 110.7773 \\
\hline 10 & 11 & 12 & 10 & 11 & 12 \\
\hline 121.7689 & 132.0210 & 142.7268 & 119.9339 & 132.2859 & 142.2566 \\
\hline 13 & 14 & 15 & 13 & 14 & 15 \\
\hline 153.0831 & 158.0160 & 163.9615 & 152.5569 & 158.0828 & 164.3838 \\
\hline 16 & 17 & 18 & 16 & 17 & 18 \\
\hline 169.2953 & 174.8431 & 180.6830 & 169.3178 & 176.4544 & 180.5499 \\
\hline 19 & 20 & 21 & 19 & 20 & 21 \\
\hline 194.4157 & 212.8705 & 218.9706 & 195.8217 & 210.0721 & 217.2202 \\
\hline
\end{tabular}




\begin{tabular}{|c|c|c|c|c|c|}
\hline 22 & 23 & 24 & 22 & 23 & 24 \\
\hline 229.0147 & 242.3066 & 246.4397 & 229.3100 & 241.9646 & 245.9502 \\
\hline 25 & 26 & 27 & 25 & 26 & 27 \\
\hline 249.0969 & 257.3797 & 268.4749 & 250.2275 & 257.3121 & 268.9294 \\
\hline 28 & 29 & 30 & 28 & 29 & 30 \\
\hline 274.7945 & 276.5920 & 283.9633 & 276.3230 & 277.8085 & 285.0440 \\
\hline 31 & 32 & 33 & 31 & 32 & 33 \\
\hline 291.6673 & 298.1221 & 308.9518 & 290.7312 & 307.9226 & 312.0398 \\
\hline 34 & 35 & 36 & 34 & 35 & 36 \\
\hline 312.4853 & 322.5757 & 327.0710 & 322.2012 & 326.2901 & 330.1857 \\
\hline 37 & 38 & 39 & 37 & 38 & 39 \\
\hline 331.2999 & 345.5730 & 360.5436 & 334.8373 & 345.8800 & 359.6556 \\
\hline 40 & 41 & 42 & 40 & 41 & 42 \\
\hline 364.1095 & 369.1509 & 397.9447 & 362.9804 & 369.2188 & 397.5475 \\
\hline 43 & 44 & 45 & 43 & 44 & 45 \\
\hline 414.4013 & 424.4309 & 450.6707 & 414.8092 & 424.3973 & 451.4050 \\
\hline 46 & 47 & 48 & 46 & 47 & 48 \\
\hline 461.1929 & 464.7882 & 475.9753 & 463.4523 & 466.5781 & 477.4620 \\
\hline 49 & 50 & 51 & 49 & 50 & 51 \\
\hline 487.9445 & 512.8143 & 523.1993 & 489.5224 & 515.6222 & 522.8478 \\
\hline 52 & 53 & 54 & 52 & 53 & 54 \\
\hline 538.3379 & 543.8334 & 554.0523 & 537.9977 & 542.4586 & 553.8013 \\
\hline 55 & 56 & 57 & 55 & 56 & 57 \\
\hline 558.8694 & 585.9058 & 590.5402 & 559.0269 & 586.4723 & 590.0815 \\
\hline 58 & 59 & 60 & 58 & 59 & 60 \\
\hline 601.7925 & 619.8794 & 625.6250 & 601.2140 & 620.1813 & 625.8483 \\
\hline 61 & 62 & 63 & 61 & 62 & 63 \\
\hline 632.3113 & 654.0869 & 662.1488 & 632.8789 & 653.6222 & 662.2425 \\
\hline 64 & 65 & 66 & 64 & 65 & 66 \\
\hline 674.6248 & 683.9204 & 699.7585 & 673.5987 & 684.1770 & 697.2070 \\
\hline 67 & 68 & 69 & 67 & 68 & 69 \\
\hline 714.3622 & 739.2296 & 750.0410 & 713.1096 & 738.6271 & 750.0895 \\
\hline 70 & 71 & 72 & 70 & 71 & 72 \\
\hline 757.7923 & 766.9939 & 778.2457 & 757.6131 & 766.9883 & 776.4065 \\
\hline 73 & 74 & 75 & 73 & 74 & 75 \\
\hline 787.9221 & 789.8352 & 826.9953 & 787.6789 & 789.5937 & 827.0014 \\
\hline 76 & 77 & 78 & 76 & 77 & 78 \\
\hline 838.3807 & 845.9093 & 851.8511 & 837.4755 & 846.4396 & 851.9447 \\
\hline 79 & 80 & 81 & 79 & 80 & 81 \\
\hline 866.2376 & 879.9098 & 889.3278 & 864.4933 & 879.4965 & 889.2600 \\
\hline 82 & 83 & 84 & 82 & 83 & 84 \\
\hline 895.8939 & 912.7562 & 921.2795 & 894.8905 & 907.9595 & 920.1091 \\
\hline 85 & 86 & 87 & 85 & 86 & 87 \\
\hline
\end{tabular}




\begin{tabular}{|c|c|c|c|c|c|}
\hline 939.6273 & 948.3912 & 952.9531 & 937.3151 & 948.0276 & 949.6068 \\
\hline 88 & 89 & 90 & 88 & 89 & 90 \\
\hline 953.2294 & 960.3358 & 964.9385 & 952.9518 & 960.6792 & 964.5943 \\
\hline 91 & 92 & 93 & 91 & 92 & 93 \\
\hline 981.5700 & 985.4657 & 993.6671 & 979.4387 & 982.8468 & 993.7983 \\
\hline 94 & 95 & 96 & 94 & 95 & 96 \\
\hline 999.7694 & 1011.6709 & 1017.0513 & 999.7888 & 1012.4076 & 1018.4437 \\
\hline 97 & 98 & 99 & 97 & 98 & 99 \\
\hline 1024.6692 & 1030.5261 & 1035.0022 & 1024.4452 & 1030.4015 & 1034.8788 \\
\hline 100 & 101 & 102 & 100 & 101 & 102 \\
\hline 1041.8395 & 1047.1502 & 1049.7500 & 1041.5847 & 1046.1700 & 1049.1901 \\
\hline 103 & 104 & 105 & 103 & 104 & 105 \\
\hline 1058.2135 & 1067.1860 & 1077.3565 & 1059.3557 & 1067.1371 & 1077.4506 \\
\hline 106 & 107 & 108 & 106 & 107 & 108 \\
\hline 1087.1273 & 1094.9568 & 1100.2567 & 1088.2696 & 1094.7368 & 1101.8880 \\
\hline 109 & 110 & 111 & 109 & 110 & 111 \\
\hline 1109.5190 & 1113.6455 & 1123.6457 & 1107.5670 & 1113.5682 & 1123.2596 \\
\hline 112 & 113 & 114 & 112 & 113 & 114 \\
\hline 1133.2929 & 1144.3061 & 1155.1045 & 1130.5695 & 1144.4978 & 1156.2465 \\
\hline 115 & 116 & 117 & 115 & 116 & 117 \\
\hline 1159.8287 & 1171.6535 & 1173.9718 & 1159.8896 & 1172.2636 & 1173.0779 \\
\hline 118 & 119 & 120 & 118 & 119 & 120 \\
\hline 1180.5738 & 1192.8724 & 1203.5063 & 1183.1443 & 1193.0020 & 1197.1834 \\
\hline 121 & 122 & 123 & 121 & 122 & 123 \\
\hline 1208.1430 & 1210.0237 & 1217.9248 & 1208.0185 & 1209.7391 & 1215.5721 \\
\hline 124 & 125 & 126 & 124 & 125 & 126 \\
\hline 1226.9585 & 1231.4603 & 1235.8193 & 1226.9818 & 1230.9281 & 1233.9680 \\
\hline 127 & 128 & 129 & 127 & 128 & 129 \\
\hline 1245.9183 & 1265.4146 & 1273.2842 & 1244.9378 & 1258.3646 & 1266.2659 \\
\hline 130 & 131 & 132 & 130 & 131 & 132 \\
\hline 1282.4168 & 1284.4116 & 1308.2788 & 1279.0269 & 1284.4515 & 1312.3498 \\
\hline 133 & 134 & 135 & 133 & 134 & 135 \\
\hline 1314.6045 & 1319.2660 & 1329.9451 & 1315.0651 & 1320.2389 & 1330.6701 \\
\hline 136 & 137 & 138 & 136 & 137 & 138 \\
\hline 1333.8301 & 1334.3432 & 1337.3854 & 1333.9015 & 1335.4122 & 1337.1982 \\
\hline 139 & 140 & 141 & 139 & 140 & 141 \\
\hline 1340.7364 & 1349.1423 & 1350.4003 & 1341.2359 & 1348.0413 & 1351.8426 \\
\hline 142 & 143 & 144 & 142 & 143 & 144 \\
\hline 1357.9376 & 1362.5777 & 1366.1491 & 1358.0611 & 1362.9378 & 1372.6751 \\
\hline 145 & 146 & 147 & 145 & 146 & 147 \\
\hline 1376.2805 & 1380.4306 & 1384.7300 & 1377.1830 & 1384.9177 & 1385.7616 \\
\hline 148 & 149 & 150 & 148 & 149 & 150 \\
\hline 1388.8154 & 1393.2946 & 1398.9424 & 1392.5598 & 1398.8896 & 1402.1432 \\
\hline
\end{tabular}




\begin{tabular}{|c|c|c|c|c|c|}
\hline 151 & 152 & 153 & 151 & 152 & 153 \\
\hline 1408.0621 & 1425.7890 & 1430.5671 & 1407.3491 & 1415.5639 & 1425.9049 \\
\hline 154 & 155 & 156 & 154 & 155 & 156 \\
\hline 1431.0367 & 1438.0906 & 1445.9613 & 1431.0817 & 1436.2190 & 1445.6240 \\
\hline 157 & 158 & 159 & 157 & 158 & 159 \\
\hline 1446.2079 & 1452.0383 & 1462.0119 & 1446.0891 & 1452.1178 & 1461.8756 \\
\hline 160 & 161 & 162 & 160 & 161 & 162 \\
\hline 1498.1654 & 1506.8714 & 1507.3307 & 1502.4749 & 1506.7903 & 1508.2733 \\
\hline 163 & 164 & 165 & 163 & 164 & 165 \\
\hline 1509.7406 & 1514.5855 & 1517.1463 & 1509.9808 & 1514.7305 & 1517.6157 \\
\hline 166 & 167 & 168 & 166 & 167 & 168 \\
\hline 1518.8159 & 1520.1591 & 1520.9022 & 1518.9414 & 1520.2700 & 1521.0309 \\
\hline 169 & 170 & 171 & 169 & 170 & 171 \\
\hline 1522.7666 & 1523.2480 & 1527.1108 & 1522.8479 & 1523.7998 & 1525.8726 \\
\hline 172 & 173 & 174 & 172 & 173 & 174 \\
\hline 1528.7680 & 1532.2506 & 1534.0993 & 1529.2994 & 1532.2417 & 1534.0988 \\
\hline 175 & 176 & 177 & 175 & 176 & 177 \\
\hline 1549.4576 & 1703.8639 & 1730.1257 & 1549.2274 & 1704.5037 & 1727.5478 \\
\hline 178 & 179 & 180 & 178 & 179 & 180 \\
\hline 1732.9499 & 1852.0160 & 1860.0583 & 1731.5135 & 1852.9471 & 1860.8779 \\
\hline 181 & 182 & 183 & 181 & 182 & 183 \\
\hline 2987.7360 & 3010.1476 & 3032.1876 & 3002.0990 & 3031.9543 & 3035.8058 \\
\hline 184 & 185 & 186 & 184 & 185 & 186 \\
\hline 3035.5958 & 3038.4778 & 3044.5119 & 3038.8728 & 3041.1491 & 3046.9093 \\
\hline 187 & 188 & 189 & 187 & 188 & 189 \\
\hline 3047.6565 & 3049.3833 & 3050.4398 & 3049.7407 & 3050.3698 & 3052.1291 \\
\hline 190 & 191 & 192 & 190 & 191 & 192 \\
\hline 3056.1768 & 3058.6592 & 3065.7521 & 3058.8136 & 3064.8655 & 3066.0957 \\
\hline 193 & 194 & 195 & 193 & 194 & 195 \\
\hline 3072.9401 & 3074.4808 & 3077.4227 & 3067.7875 & 3072.0747 & 3074.5248 \\
\hline 196 & 197 & 198 & 196 & 197 & 198 \\
\hline 3077.7565 & 3079.1221 & 3081.2823 & 3077.3665 & 3077.5703 & 3080.6648 \\
\hline 199 & 200 & 201 & 199 & 200 & 201 \\
\hline 3086.7538 & 3100.1783 & 3103.0299 & 3081.3279 & 3100.8729 & 3103.3039 \\
\hline 202 & 203 & 204 & 202 & 203 & 204 \\
\hline 3111.2508 & 3115.3022 & 3120.5666 & 3111.3904 & 3115.2793 & 3120.7380 \\
\hline 205 & 206 & 207 & 205 & 206 & 207 \\
\hline 3122.8071 & 3129.7585 & 3130.9347 & 3121.5391 & 3122.1774 & 3130.9167 \\
\hline 208 & 209 & 210 & 208 & 209 & 210 \\
\hline 3132.0639 & 3136.7857 & 3143.7496 & 3131.9530 & 3132.6340 & 3144.0295 \\
\hline 211 & 212 & 213 & 211 & 212 & 213 \\
\hline 3151.8099 & 3165.4309 & 3182.6421 & 3148.8294 & 3165.5903 & 3180.7038 \\
\hline 214 & 215 & 216 & 214 & 215 & 216 \\
\hline
\end{tabular}




\begin{tabular}{|c|c|c|c|c|c|}
\hline 3194.8410 & 3726.3616 & 3753.6705 & 3194.5200 & 3727.1775 & 3753.9297 \\
\hline \multicolumn{3}{|c|}{$\mathrm{C3}$} & \multicolumn{3}{|c|}{$\mathrm{C} 4$} \\
\hline 1 & 2 & 3 & 1 & 2 & 3 \\
\hline 29.2931 & 33.5849 & 42.2635 & 29.6507 & 33.0166 & 42.4421 \\
\hline 4 & 5 & 6 & 4 & 5 & 6 \\
\hline 53.7262 & 68.4688 & 77.4375 & 55.5360 & 68.7231 & 77.4379 \\
\hline 7 & 8 & 9 & 7 & 8 & 9 \\
\hline 92.5322 & 95.9211 & 111.1871 & 92.0496 & 95.7774 & 111.7827 \\
\hline 10 & 11 & 12 & 10 & 11 & 12 \\
\hline 121.6533 & 131.8068 & 142.3654 & 119.9854 & 132.1066 & 141.9854 \\
\hline 13 & 14 & 15 & 13 & 14 & 15 \\
\hline 152.7361 & 160.1446 & 164.0296 & 152.5080 & 160.2742 & 164.4438 \\
\hline 16 & 17 & 18 & 16 & 17 & 18 \\
\hline 170.3803 & 174.7480 & 178.5272 & 170.7775 & 176.3605 & 178.3030 \\
\hline 19 & 20 & 21 & 19 & 20 & 21 \\
\hline 193.0472 & 213.5545 & 217.2487 & 194.4436 & 210.0582 & 217.3292 \\
\hline 22 & 23 & 24 & 22 & 23 & 24 \\
\hline 227.5213 & 244.4967 & 247.5831 & 226.8055 & 243.6809 & 249.1984 \\
\hline 25 & 26 & 27 & 25 & 26 & 27 \\
\hline 254.5251 & 266.4723 & 270.4699 & 254.4629 & 266.1867 & 271.0880 \\
\hline 28 & 29 & 30 & 28 & 29 & 30 \\
\hline 274.7503 & 283.0455 & 284.8017 & 277.2179 & 282.5683 & 285.5897 \\
\hline 31 & 32 & 33 & 31 & 32 & 33 \\
\hline 291.5203 & 298.2455 & 308.1041 & 290.6015 & 306.8906 & 312.2914 \\
\hline 34 & 35 & 36 & 34 & 35 & 36 \\
\hline 312.5539 & 323.5576 & 327.6795 & 323.1299 & 326.6335 & 330.2495 \\
\hline 37 & 38 & 39 & 37 & 38 & 39 \\
\hline 331.3891 & 345.6981 & 360.5098 & 335.6416 & 346.1670 & 359.5847 \\
\hline 40 & 41 & 42 & 40 & 41 & 42 \\
\hline 363.9786 & 368.9295 & 398.7704 & 362.8450 & 368.9925 & 398.3486 \\
\hline 43 & 44 & 45 & 43 & 44 & 45 \\
\hline 415.4194 & 421.8113 & 451.1159 & 415.7500 & 421.7527 & 451.8414 \\
\hline 46 & 47 & 48 & 46 & 47 & 48 \\
\hline 461.4367 & 464.9182 & 478.5998 & 463.8297 & 466.7354 & 479.9954 \\
\hline 49 & 50 & 51 & 49 & 50 & 51 \\
\hline 487.8362 & 512.9689 & 522.9033 & 489.3547 & 515.7795 & 522.5850 \\
\hline 52 & 53 & 54 & 52 & 53 & 54 \\
\hline 537.8327 & 544.4397 & 554.5691 & 537.3551 & 543.1035 & 554.3164 \\
\hline 55 & 56 & 57 & 55 & 56 & 57 \\
\hline 557.4578 & 586.2623 & 592.2300 & 557.5846 & 586.9390 & 591.5682 \\
\hline 58 & 59 & 60 & 58 & 59 & 60 \\
\hline 602.5206 & 619.7943 & 626.5172 & 602.0757 & 620.0807 & 626.7617 \\
\hline
\end{tabular}




\begin{tabular}{|c|c|c|c|c|c|}
\hline 61 & 62 & 63 & 61 & 62 & 63 \\
\hline 632.4243 & 654.2356 & 664.1830 & 632.9459 & 653.7983 & 664.2807 \\
\hline 64 & 65 & 66 & 64 & 65 & 66 \\
\hline 674.9501 & 684.1404 & 700.2519 & 673.8147 & 684.3446 & 697.6671 \\
\hline 67 & 68 & 69 & 67 & 68 & 69 \\
\hline 715.6154 & 738.5013 & 750.2795 & 714.5693 & 737.8893 & 750.3675 \\
\hline 70 & 71 & 72 & 70 & 71 & 72 \\
\hline 757.6304 & 766.2621 & 778.2501 & 757.4531 & 766.3113 & 776.3247 \\
\hline 73 & 74 & 75 & 73 & 74 & 75 \\
\hline 787.5488 & 790.4103 & 827.1850 & 787.3989 & 790.0548 & 827.1840 \\
\hline 76 & 77 & 78 & 76 & 77 & 78 \\
\hline 839.0547 & 845.0669 & 851.4579 & 838.1939 & 845.4558 & 851.7487 \\
\hline 79 & 80 & 81 & 79 & 80 & 81 \\
\hline 865.7227 & 878.7030 & 886.3946 & 864.0035 & 878.3890 & 886.1335 \\
\hline 82 & 83 & 84 & 82 & 83 & 84 \\
\hline 895.6892 & 912.5089 & 921.2446 & 894.8708 & 907.7659 & 920.0771 \\
\hline 85 & 86 & 87 & 85 & 86 & 87 \\
\hline 939.4799 & 947.8533 & 953.0470 & 937.2393 & 947.4668 & 949.5200 \\
\hline 88 & 89 & 90 & 88 & 89 & 90 \\
\hline 953.9850 & 960.1448 & 963.3283 & 953.9701 & 960.4766 & 962.9029 \\
\hline 91 & 92 & 93 & 91 & 92 & 93 \\
\hline 981.8066 & 985.3406 & 994.9516 & 979.5607 & 982.7884 & 995.1034 \\
\hline 94 & 95 & 96 & 94 & 95 & 96 \\
\hline 999.7750 & 1011.7906 & 1016.5369 & 999.8878 & 1012.7415 & 1017.1240 \\
\hline 97 & 98 & 99 & 97 & 98 & 99 \\
\hline 1020.1053 & 1027.4657 & 1037.4292 & 1020.5120 & 1027.1833 & 1037.1358 \\
\hline 100 & 101 & 102 & 100 & 101 & 102 \\
\hline 1042.8344 & 1047.8645 & 1053.2964 & 1042.7839 & 1046.4610 & 1053.1653 \\
\hline 103 & 104 & 105 & 103 & 104 & 105 \\
\hline 1058.2069 & 1066.0115 & 1077.2838 & 1059.3224 & 1065.9762 & 1077.4265 \\
\hline 106 & 107 & 108 & 106 & 107 & 108 \\
\hline 1087.0747 & 1094.8471 & 1100.3302 & 1088.2410 & 1094.6502 & 1101.6716 \\
\hline 109 & 110 & 111 & 109 & 110 & 111 \\
\hline 1106.8867 & 1110.9345 & 1121.8475 & 1107.2947 & 1108.5855 & 1121.7068 \\
\hline 112 & 113 & 114 & 112 & 113 & 114 \\
\hline 1133.0578 & 1139.2620 & 1155.0892 & 1130.2552 & 1139.0893 & 1156.3601 \\
\hline 115 & 116 & 117 & 115 & 116 & 117 \\
\hline 1160.7715 & 1171.9397 & 1174.5692 & 1160.8414 & 1172.6655 & 1173.4781 \\
\hline 118 & 119 & 120 & 118 & 119 & 120 \\
\hline 1180.7717 & 1192.3663 & 1196.6022 & 1183.2411 & 1192.5029 & 1196.6323 \\
\hline 121 & 122 & 123 & 121 & 122 & 123 \\
\hline 1203.5867 & 1210.8565 & 1217.3384 & 1197.3564 & 1210.4842 & 1214.9722 \\
\hline 124 & 125 & 126 & 124 & 125 & 126 \\
\hline
\end{tabular}




\begin{tabular}{|c|c|c|c|c|c|}
\hline 1228.0029 & 1235.7128 & 1238.1807 & 1227.5737 & 1233.6436 & 1238.0067 \\
\hline 127 & 128 & 129 & 127 & 128 & 129 \\
\hline 1246.3252 & 1273.0869 & 1278.6541 & 1245.2991 & 1258.8430 & 1278.7203 \\
\hline 130 & 131 & 132 & 130 & 131 & 132 \\
\hline 1281.8239 & 1290.5526 & 1309.2603 & 1278.9131 & 1290.1450 & 1314.9385 \\
\hline 133 & 134 & 135 & 133 & 134 & 135 \\
\hline 1315.3017 & 1318.6292 & 1327.7626 & 1315.3210 & 1318.3992 & 1328.4449 \\
\hline 136 & 137 & 138 & 136 & 137 & 138 \\
\hline 1330.1288 & 1334.2478 & 1337.2800 & 1330.4525 & 1335.3415 & 1337.2583 \\
\hline 139 & 140 & 141 & 139 & 140 & 141 \\
\hline 1341.5513 & 1349.1445 & 1350.3076 & 1341.8403 & 1347.8925 & 1351.8684 \\
\hline 142 & 143 & 144 & 142 & 143 & 144 \\
\hline 1360.4067 & 1365.4695 & 1374.0030 & 1360.6768 & 1371.6393 & 1374.4427 \\
\hline 145 & 146 & 147 & 145 & 146 & 147 \\
\hline 1376.3521 & 1380.2338 & 1383.9019 & 1377.6880 & 1383.2194 & 1385.5619 \\
\hline 148 & 149 & 150 & 148 & 149 & 150 \\
\hline 1386.3932 & 1390.7234 & 1396.0062 & 1388.3701 & 1395.9568 & 1400.8418 \\
\hline 151 & 152 & 153 & 151 & 152 & 153 \\
\hline 1401.0974 & 1408.2563 & 1430.7414 & 1402.0242 & 1407.5895 & 1415.6943 \\
\hline 154 & 155 & 156 & 154 & 155 & 156 \\
\hline 1438.1263 & 1441.5255 & 1446.0209 & 1436.3748 & 1441.5342 & 1445.6959 \\
\hline 157 & 158 & 159 & 157 & 158 & 159 \\
\hline 1446.2164 & 1452.2537 & 1462.1858 & 1446.0974 & 1452.3776 & 1462.0787 \\
\hline 160 & 161 & 162 & 160 & 161 & 162 \\
\hline 1498.1623 & 1507.1810 & 1507.4525 & 1502.4416 & 1507.2638 & 1508.2340 \\
\hline 163 & 164 & 165 & 163 & 164 & 165 \\
\hline 1509.7546 & 1514.7703 & 1517.1463 & 1509.9771 & 1514.9558 & 1517.6209 \\
\hline 166 & 167 & 168 & 166 & 167 & 168 \\
\hline 1518.8840 & 1520.3716 & 1521.1607 & 1519.0202 & 1520.6408 & 1521.2658 \\
\hline 169 & 170 & 171 & 169 & 170 & 171 \\
\hline 1523.2514 & 1523.9230 & 1527.1211 & 1523.7620 & 1523.9268 & 1525.9003 \\
\hline 172 & 173 & 174 & 172 & 173 & 174 \\
\hline 1528.7789 & 1532.8862 & 1534.5585 & 1529.2474 & 1532.9475 & 1534.6162 \\
\hline 175 & 176 & 177 & 175 & 176 & 177 \\
\hline 1551.1332 & 1704.3237 & 1730.0775 & 1550.8844 & 1705.0086 & 1727.5024 \\
\hline 178 & 179 & 180 & 178 & 179 & 180 \\
\hline 1732.8418 & 1853.9087 & 1860.4505 & 1731.4639 & 1854.8238 & 1861.2690 \\
\hline 181 & 182 & 183 & 181 & 182 & 183 \\
\hline 2988.2970 & 3010.2176 & 3032.1284 & 3002.1742 & 3031.8828 & 3041.4367 \\
\hline 184 & 185 & 186 & 184 & 185 & 186 \\
\hline 3043.5037 & 3044.3806 & 3044.6075 & 3043.6327 & 3044.5604 & 3047.0347 \\
\hline 187 & 188 & 189 & 187 & 188 & 189 \\
\hline 3047.4747 & 3048.3404 & 3055.0071 & 3048.6104 & 3050.0793 & 3056.5405 \\
\hline
\end{tabular}




\begin{tabular}{|c|c|c|c|c|c|}
\hline 190 & 191 & 192 & 190 & 191 & 192 \\
\hline 3056.2271 & 3058.9929 & 3069.2611 & 3059.1224 & 3065.0038 & 3068.0989 \\
\hline 193 & 194 & 195 & 193 & 194 & 195 \\
\hline 3073.6704 & 3074.8617 & 3077.5743 & 3069.5344 & 3072.8341 & 3074.8865 \\
\hline 196 & 197 & 198 & 196 & 197 & 198 \\
\hline 3077.7670 & 3079.2204 & 3083.6497 & 3077.2101 & 3077.9261 & 3080.7612 \\
\hline 199 & 200 & 201 & 199 & 200 & 201 \\
\hline 3086.9514 & 3099.4621 & 3099.8195 & 3083.7182 & 3100.2005 & 3100.5026 \\
\hline 202 & 203 & 204 & 202 & 203 & 204 \\
\hline 3110.1377 & 3111.3935 & 3115.9766 & 3110.5600 & 3111.5055 & 3115.9451 \\
\hline 205 & 206 & 207 & 205 & 206 & 207 \\
\hline 3121.0592 & 3122.8369 & 3130.0603 & 3121.2471 & 3121.5133 & 3122.2268 \\
\hline 208 & 209 & 210 & 208 & 209 & 210 \\
\hline 3131.0477 & 3136.9197 & 3142.1841 & 3131.1754 & 3132.1198 & 3142.3757 \\
\hline 211 & 212 & 213 & 211 & 212 & 213 \\
\hline 3151.9582 & 3158.7334 & 3181.1353 & 3149.0149 & 3158.8084 & 3179.1935 \\
\hline 214 & 215 & 216 & 214 & 215 & 216 \\
\hline 3194.8751 & 3726.4441 & 3762.7291 & 3194.6074 & 3727.0631 & 3762.9466 \\
\hline
\end{tabular}

Table S11. Frequencies data for 2.

\begin{tabular}{|c|c|c|c|c|c|c|c|c|}
\hline \multicolumn{3}{|c|}{ C1 } & \multicolumn{3}{|c|}{ C2 } & \multicolumn{3}{|c|}{$\mathrm{C} 3$} \\
\hline 1 & 2 & 3 & 1 & 2 & 3 & 1 & 2 & 3 \\
\hline 28.7118 & 37.5679 & 50.5547 & 30.4886 & 40.5042 & 51.3947 & 29.3349 & 48.6499 & 60.9378 \\
\hline 4 & 5 & 6 & 4 & 5 & 6 & 4 & 5 & 6 \\
\hline 61.4120 & 75.1608 & 79.1025 & 65.7362 & 76.8447 & 80.7989 & 67.5898 & 77.7725 & 88.6307 \\
\hline 7 & 8 & 9 & 7 & 8 & 9 & 7 & 8 & 9 \\
\hline 84.2266 & 95.9291 & 106.7292 & 85.8591 & 99.6307 & 108.9892 & 94.2878 & 104.6970 & 107.9585 \\
\hline 10 & 11 & 12 & 10 & 11 & 12 & 10 & 11 & 12 \\
\hline 118.4062 & 134.5242 & 139.8317 & 120.3972 & 134.7458 & 139.6013 & 134.2872 & 147.9547 & 152.2897 \\
\hline 13 & 14 & 15 & 13 & 14 & 15 & 13 & 14 & 15 \\
\hline 158.5098 & 167.7058 & 169.7757 & 158.3259 & 168.5466 & 173.1650 & 160.5303 & 171.2485 & 179.7350 \\
\hline 16 & 17 & 18 & 16 & 17 & 18 & 16 & 17 & 18 \\
\hline 175.0101 & 184.6582 & 194.4280 & 183.9879 & 194.6688 & 199.5340 & 192.1347 & 198.5756 & 202.7619 \\
\hline 19 & 20 & 21 & 19 & 20 & 21 & 19 & 20 & 21 \\
\hline 199.6490 & 206.1890 & 226.5858 & 206.3414 & 225.5571 & 247.3072 & 209.1320 & 214.0724 & 227.2824 \\
\hline 22 & 23 & 24 & 22 & 23 & 24 & 22 & 23 & 24 \\
\hline 245.6678 & 250.5665 & 258.1328 & 251.6731 & 258.8866 & 269.6266 & 239.1441 & 245.9387 & 253.7606 \\
\hline 25 & 26 & 27 & 25 & 26 & 27 & 25 & 26 & 27 \\
\hline 269.6921 & 279.6103 & 282.5609 & 280.2947 & 283.0638 & 286.7024 & 259.8985 & 272.0308 & 281.7352 \\
\hline 28 & 29 & 30 & 28 & 29 & 30 & 28 & 29 & 30 \\
\hline 289.9843 & 297.0100 & 307.2791 & 298.3338 & 306.5408 & 308.7619 & 288.2543 & 295.7493 & 308.3897 \\
\hline 31 & 32 & 33 & 31 & 32 & 33 & 31 & 32 & 33 \\
\hline 310.2264 & 321.8167 & 329.5567 & 318.5272 & 324.7635 & 330.4587 & 322.6571 & 335.8729 & 351.4930 \\
\hline
\end{tabular}




\begin{tabular}{|c|c|c|c|c|c|c|c|c|}
\hline 34 & 35 & 36 & 34 & 35 & 36 & 34 & 35 & 36 \\
\hline 345.0055 & 365.0815 & 369.2749 & 345.5490 & 364.7164 & 369.1450 & 367.0417 & 368.5322 & 384.4567 \\
\hline 37 & 38 & 39 & 37 & 38 & 39 & 37 & 38 & 39 \\
\hline 389.7472 & 401.5768 & 414.1237 & 390.2057 & 401.5279 & 413.8520 & 403.4192 & 426.8964 & 427.7586 \\
\hline 40 & 41 & 42 & 40 & 41 & 42 & 40 & 41 & 42 \\
\hline 424.0535 & 428.4632 & 440.0812 & 424.1772 & 428.8997 & 440.1731 & 436.4984 & 443.6158 & 461.9316 \\
\hline 43 & 44 & 45 & 43 & 44 & 45 & 43 & 44 & 45 \\
\hline 465.3663 & 468.7963 & 483.7305 & 465.2588 & 467.7950 & 483.5116 & 470.1110 & 485.2319 & 505.3469 \\
\hline 46 & 47 & 48 & 46 & 47 & 48 & 46 & 47 & 48 \\
\hline 501.9551 & 528.4592 & 532.7970 & 501.2713 & 527.6928 & 532.8314 & 530.4924 & 534.4168 & 542.9397 \\
\hline 49 & 50 & 51 & 49 & 50 & 51 & 49 & 50 & 51 \\
\hline 539.3186 & 557.2865 & 581.5805 & 540.1491 & 557.5138 & 583.6917 & 553.1576 & 574.3865 & 583.9492 \\
\hline 52 & 53 & 54 & 52 & 53 & 54 & 52 & 53 & 54 \\
\hline 596.4985 & 608.6840 & 615.6619 & 598.6885 & 608.3577 & 616.0742 & 592.1451 & 607.8642 & 615.2081 \\
\hline 55 & 56 & 57 & 55 & 56 & 57 & 55 & 56 & 57 \\
\hline 627.1716 & 634.8489 & 648.5280 & 627.9438 & 634.8194 & 648.9498 & 628.6710 & 633.3382 & 645.7439 \\
\hline 58 & 59 & 60 & 58 & 59 & 60 & 58 & 59 & 60 \\
\hline 659.2605 & 669.2526 & 679.8633 & 659.6952 & 670.9210 & 681.7059 & 668.8441 & 677.5499 & 692.3245 \\
\hline 61 & 62 & 63 & 61 & 62 & 63 & 61 & 62 & 63 \\
\hline 704.5337 & 708.0118 & 722.1682 & 705.0579 & 708.5432 & 722.7499 & 705.4124 & 708.6666 & 721.1519 \\
\hline 64 & 65 & 66 & 64 & 65 & 66 & 64 & 65 & 66 \\
\hline 739.9735 & 752.8499 & 758.4280 & 740.0819 & 752.9864 & 758.4618 & 738.8735 & 753.6034 & 759.1952 \\
\hline 67 & 68 & 69 & 67 & 68 & 69 & 67 & 68 & 69 \\
\hline 767.7807 & 779.9866 & 802.8206 & 767.6703 & 780.8892 & 803.3315 & 771.1830 & 777.4633 & 795.7181 \\
\hline 70 & 71 & 72 & 70 & 71 & 72 & 70 & 71 & 72 \\
\hline 810.4720 & 830.1096 & 834.1573 & 810.8752 & 829.6639 & 833.5213 & 807.8742 & 830.1770 & 840.5361 \\
\hline 73 & 74 & 75 & 73 & 74 & 75 & 73 & 74 & 75 \\
\hline 853.5980 & 868.2434 & 877.3012 & 853.0464 & 867.5597 & 873.4034 & 844.7430 & 862.2170 & 867.2837 \\
\hline 76 & 77 & 78 & 76 & 77 & 78 & 76 & 77 & 78 \\
\hline 884.0375 & 901.7314 & 915.5004 & 884.4380 & 901.9489 & 916.0573 & 879.6646 & 901.7932 & 916.2476 \\
\hline 79 & 80 & 81 & 79 & 80 & 81 & 79 & 80 & 81 \\
\hline 939.0260 & 950.2638 & 952.3427 & 938.8673 & 950.2250 & 952.7544 & 935.7874 & 946.8919 & 951.0521 \\
\hline 82 & 83 & 84 & 82 & 83 & 84 & 82 & 83 & 84 \\
\hline 957.8179 & 968.1035 & 973.0437 & 958.5233 & 969.9740 & 973.1397 & 958.4750 & 964.6049 & 970.5289 \\
\hline 85 & 86 & 87 & 85 & 86 & 87 & 85 & 86 & 87 \\
\hline 983.5784 & 987.8045 & 1005.3307 & 984.5563 & 988.3444 & 1003.7150 & 981.3154 & 985.6295 & 1005.2521 \\
\hline 88 & 89 & 90 & 88 & 89 & 90 & 88 & 89 & 90 \\
\hline 1012.5170 & 1017.6076 & 1019.9928 & 1010.2872 & 1014.1496 & 1021.2719 & 1008.0832 & 1018.5044 & 1024.3845 \\
\hline 91 & 92 & 93 & 91 & 92 & 93 & 91 & 92 & 93 \\
\hline 1032.4620 & 1039.2047 & 1045.3925 & 1031.9598 & 1039.1369 & 1048.8152 & 1031.5838 & 1035.9729 & 1043.0435 \\
\hline 94 & 95 & 96 & 94 & 95 & 96 & 94 & 95 & 96 \\
\hline 1049.7943 & 1057.7774 & 1061.3388 & 1055.1891 & 1057.7335 & 1061.3855 & 1047.6399 & 1057.9180 & 1062.1599 \\
\hline 97 & 98 & 99 & 97 & 98 & 99 & 97 & 98 & 99 \\
\hline
\end{tabular}




\begin{tabular}{|c|c|c|c|c|c|c|c|c|}
\hline 1065.7420 & 1073.2977 & 1074.4418 & 1066.7751 & 1073.4513 & 1074.4714 & 1064.3532 & 1072.0846 & 1073.4457 \\
\hline 100 & 101 & 102 & 100 & 101 & 102 & 100 & 101 & 102 \\
\hline 1077.9812 & 1083.3329 & 1091.1675 & 1078.2955 & 1081.0211 & 1091.2690 & 1075.3749 & 1081.0811 & 1088.0978 \\
\hline 103 & 104 & 105 & 103 & 104 & 105 & 103 & 104 & 105 \\
\hline 1099.5587 & 1111.7390 & 1114.3150 & 1106.7096 & 1108.6462 & 1111.7034 & 1098.7940 & 1110.3431 & 1112.5989 \\
\hline 106 & 107 & 108 & 106 & 107 & 108 & 106 & 107 & 108 \\
\hline 1115.5567 & 1128.8198 & 1143.2202 & 1114.5995 & 1129.4576 & 1145.9457 & 1115.4534 & 1126.0652 & 1144.0107 \\
\hline 109 & 110 & 111 & 109 & 110 & 111 & 109 & 110 & 111 \\
\hline 1152.5005 & 1156.1505 & 1170.3642 & 1152.3522 & 1156.0019 & 1170.6305 & 1151.6536 & 1156.0067 & 1171.4278 \\
\hline 112 & 113 & 114 & 112 & 113 & 114 & 112 & 113 & 114 \\
\hline 1181.6098 & 1186.3439 & 1191.8781 & 1179.8695 & 1185.9228 & 1191.1310 & 1181.0426 & 1186.3490 & 1192.1155 \\
\hline 115 & 116 & 117 & 115 & 116 & 117 & 115 & 116 & 117 \\
\hline 1206.6250 & 1212.4335 & 1220.9344 & 1207.9878 & 1212.3242 & 1218.3296 & 1206.1531 & 1212.8618 & 1220.1841 \\
\hline 118 & 119 & 120 & 118 & 119 & 120 & 118 & 119 & 120 \\
\hline 1223.8183 & 1232.6059 & 1235.9636 & 1221.4569 & 1230.4192 & 1232.6820 & 1223.0108 & 1232.5588 & 1236.4605 \\
\hline 121 & 122 & 123 & 121 & 122 & 123 & 121 & 122 & 123 \\
\hline 1243.6591 & 1250.0445 & 1255.6716 & 1240.7913 & 1250.3625 & 1261.5134 & 1240.7099 & 1243.5145 & 1253.1069 \\
\hline 124 & 125 & 126 & 124 & 125 & 126 & 124 & 125 & 126 \\
\hline 1263.0706 & 1266.5312 & 1269.5512 & 1263.2329 & 1269.5750 & 1279.7778 & 1264.4372 & 1270.9369 & 1291.2080 \\
\hline 127 & 128 & 129 & 127 & 128 & 129 & 127 & 128 & 129 \\
\hline 1287.7640 & 1299.0902 & 1308.8110 & 1290.9329 & 1299.3408 & 1306.8300 & 1297.7229 & 1307.1147 & 1308.6404 \\
\hline 130 & 131 & 132 & 130 & 131 & 132 & 130 & 131 & 132 \\
\hline 1312.2152 & 1322.8832 & 1328.1155 & 1308.6934 & 1322.6413 & 1326.5407 & 1321.6147 & 1331.4562 & 1333.6659 \\
\hline 133 & 134 & 135 & 133 & 134 & 135 & 133 & 134 & 135 \\
\hline 1330.3692 & 1334.9459 & 1344.9405 & 1330.1736 & 1334.2792 & 1348.9830 & 1349.4484 & 1354.4112 & 1356.0933 \\
\hline 136 & 137 & 138 & 136 & 137 & 138 & 136 & 137 & 138 \\
\hline 1348.9537 & 1357.0986 & 1374.3021 & 1356.9964 & 1359.1484 & 1375.5050 & 1359.5369 & 1374.5925 & 1377.3897 \\
\hline 139 & 140 & 141 & 139 & 140 & 141 & 139 & 140 & 141 \\
\hline 1377.6125 & 1379.9331 & 1382.7948 & 1377.4792 & 1378.9147 & 1382.8195 & 1382.7686 & 1386.6918 & 1391.4009 \\
\hline 142 & 143 & 144 & 142 & 143 & 144 & 142 & 143 & 144 \\
\hline 1398.7236 & 1408.8234 & 1409.4799 & 1396.5926 & 1407.9796 & 1408.9011 & 1401.1792 & 1409.6038 & 1415.8752 \\
\hline 145 & 146 & 147 & 145 & 146 & 147 & 145 & 146 & 147 \\
\hline 1418.7026 & 1436.1699 & 1442.3133 & 1417.9093 & 1439.7670 & 1442.3841 & 1419.6564 & 1432.9066 & 1441.0470 \\
\hline 148 & 149 & 150 & 148 & 149 & 150 & 148 & 149 & 150 \\
\hline 1448.0757 & 1461.8211 & 1473.0549 & 1448.3862 & 1454.2216 & 1473.0623 & 1442.9482 & 1458.9147 & 1467.5373 \\
\hline 151 & 152 & 153 & 151 & 152 & 153 & 151 & 152 & 153 \\
\hline 1483.3984 & 1500.2955 & 1502.7998 & 1483.9565 & 1500.3863 & 1503.2451 & 1473.6646 & 1504.9792 & 1505.8200 \\
\hline 154 & 155 & 156 & 154 & 155 & 156 & 154 & 155 & 156 \\
\hline 1512.8124 & 1522.4669 & 1523.6342 & 1512.9450 & 1523.2363 & 1525.0341 & 1512.8375 & 1520.5665 & 1521.3397 \\
\hline 157 & 158 & 159 & 157 & 158 & 159 & 157 & 158 & 159 \\
\hline 1524.9657 & 1526.4996 & 1530.7130 & 1525.6957 & 1526.5168 & 1531.5750 & 1523.5990 & 1524.0102 & 1527.039 \\
\hline 160 & 161 & 162 & 160 & 161 & 162 & 160 & 161 & 162 \\
\hline 1534.2841 & 1535.6739 & 1542.9900 & 1533.5860 & 1538.2319 & 1542.6675 & 1528.5613 & 1529.0491 & 1534.7517 \\
\hline
\end{tabular}




\begin{tabular}{|c|c|c|c|c|c|c|c|c|}
\hline 163 & 164 & 165 & 163 & 164 & 165 & 163 & 164 & 165 \\
\hline 1552.0077 & 1553.0990 & 1696.2126 & 1552.0184 & 1552.7348 & 1695.9128 & 1551.2315 & 1557.3099 & 1690.4960 \\
\hline 166 & 167 & 168 & 166 & 167 & 168 & 166 & 167 & 168 \\
\hline 1725.1988 & 1741.9957 & 1754.0948 & 1725.1861 & 1742.0945 & 1753.4426 & 1726.3206 & 1742.2926 & 1753.4043 \\
\hline 169 & 170 & 171 & 169 & 170 & 171 & 169 & 170 & 171 \\
\hline 1835.3608 & 1857.9182 & 2963.8620 & 1836.4770 & 1859.6391 & 2963.0534 & 1807.6423 & 1858.3151 & 2966.5232 \\
\hline 172 & 173 & 174 & 172 & 173 & 174 & 172 & 173 & 174 \\
\hline 2979.0025 & 3013.6610 & 3032.7164 & 2979.1136 & 3012.8041 & 3033.6058 & 2990.4002 & 3018.0134 & 3032.6199 \\
\hline 175 & 176 & 177 & 175 & 176 & 177 & 175 & 176 & 177 \\
\hline 3034.9791 & 3036.1487 & 3038.3220 & 3036.3638 & 3037.4766 & 3038.8727 & 3036.3590 & 3039.1210 & 3043.1707 \\
\hline 178 & 179 & 180 & 178 & 179 & 180 & 178 & 179 & 180 \\
\hline 3043.2804 & 3055.5847 & 3063.2681 & 3042.5493 & 3063.1991 & 3063.2532 & 3049.5004 & 3057.7506 & 3061.5341 \\
\hline 181 & 182 & 183 & 181 & 182 & 183 & 181 & 182 & 183 \\
\hline 3064.0626 & 3072.5381 & 3076.1451 & 3069.5089 & 3073.4379 & 3075.3898 & 3064.9666 & 3065.0201 & 3076.9834 \\
\hline 184 & 185 & 186 & 184 & 185 & 186 & 184 & 185 & 186 \\
\hline 3077.4758 & 3082.1352 & 3089.2981 & 3077.5765 & 3079.7644 & 3081.9987 & 3082.0464 & 3084.4144 & 3087.1496 \\
\hline 187 & 188 & 189 & 187 & 188 & 189 & 187 & 188 & 189 \\
\hline 3093.6942 & 3105.0407 & 3109.3471 & 3092.5840 & 3111.1328 & 3111.4778 & 3094.4028 & 3103.4213 & 3110.2374 \\
\hline 190 & 191 & 192 & 190 & 191 & 192 & 190 & 191 & 192 \\
\hline 3112.9130 & 3125.3892 & 3134.5836 & 3113.6975 & 3120.3949 & 3125.7150 & 3113.6450 & 3125.8178 & 3134.8569 \\
\hline 193 & 194 & 195 & 193 & 194 & 195 & 193 & 194 & 195 \\
\hline 3143.9426 & 3145.0681 & 3148.8909 & 3134.1133 & 3142.5312 & 3148.0151 & 3144.8040 & 3149.5702 & 3149.6911 \\
\hline 196 & 197 & 198 & 196 & 197 & 198 & 196 & 197 & 198 \\
\hline 3149.4700 & 3151.3212 & 3159.7917 & 3150.9149 & 3151.4018 & 3159.9595 & 3152.0845 & 3153.1075 & 3160.3084 \\
\hline 199 & 200 & 201 & 199 & 200 & 201 & 199 & 200 & 201 \\
\hline 3167.0629 & 3214.9058 & 3235.6371 & 3167.0877 & 3214.5017 & 3235.8458 & 3168.4399 & 3215.8125 & 3236.1539 \\
\hline 202 & 203 & 204 & 202 & 203 & 204 & 202 & 203 & 204 \\
\hline 3236.3734 & 3751.7573 & 3758.5048 & 3236.5034 & 3752.6594 & 3760.2387 & 3237.4552 & 3658.9288 & 3752.6733 \\
\hline
\end{tabular}

Table S12. Calculated ${ }^{1} \mathrm{H}-\mathrm{NMR}$ chemical shifts for conformers of $1 \mathrm{a}$ and $\mathbf{1 b}$.

\begin{tabular}{|l|l|l|l|l|l|l|}
\hline No. & \multicolumn{2}{|c|}{ 1a } & \multicolumn{4}{c|}{ 1b } \\
\hline & C1 & C2 & C1 & C2 & C3 & C4 \\
\hline 1 & 2.5484 & 2.4058 & 1.9432 & 2.4948 & 1.9453 & 2.4946 \\
\hline 2 & 2.1722 & 2.1500 & 2.1349 & 2.1518 & 2.1376 & 2.1548 \\
\hline & 2.2633 & 2.3463 & 2.3288 & 2.2442 & 2.3225 & 2.2396 \\
\hline 3 & 5.7605 & 5.7587 & 5.7436 & 5.7473 & 5.7451 & 5.7490 \\
\hline 5 & 2.5484 & 2.4058 & 2.3988 & 2.5502 & 2.3977 & 2.5471 \\
\hline 6 & 1.8957 & 1.9190 & 1.8930 & 1.8754 & 1.8734 & 1.8565 \\
\hline & 2.1633 & 2.1288 & 2.1288 & 2.1715 & 2.1106 & 2.1534 \\
\hline 9 & 2.2301 & 2.2581 & 2.2506 & 2.4975 & 2.2447 & 2.4904 \\
\hline
\end{tabular}




\begin{tabular}{|c|c|c|c|c|c|c|}
\hline & 2.4907 & 2.5758 & 2.4795 & 2.1249 & 2.4811 & 2.1275 \\
\hline \multirow[t]{2}{*}{13} & 1.8115 & 1.7768 & 1.7783 & 1.8179 & 1.7686 & 1.8085 \\
\hline & 2.6892 & 2.6729 & 2.6915 & 2.7101 & 2.6971 & 2.7154 \\
\hline \multirow[t]{3}{*}{14} & 1.8801 & 1.8794 & 1.8742 & 1.8740 & 1.8728 & 1.8731 \\
\hline & 1.5908 & 1.5635 & 1.5554 & 1.5836 & 1.5560 & 1.5840 \\
\hline & 1.6582 & 1.6932 & 1.7166 & 1.6859 & 1.7018 & 1.6717 \\
\hline \multirow[t]{3}{*}{15} & 1.1153 & 0.6294 & 0.6415 & 1.1158 & 0.6415 & 1.1160 \\
\hline & 0.8117 & 1.2989 & 1.2667 & 0.7806 & 1.2704 & 0.7826 \\
\hline & 1.2768 & 1.3548 & 1.3327 & 1.2519 & 1.3346 & 1.2542 \\
\hline 1 ' & 3.0695 & 3.0702 & 3.7436 & 3.7381 & 3.7169 & 3.7114 \\
\hline \multirow[t]{2}{*}{$2^{\prime}$} & 1.4098 & 1.4104 & 0.9619 & 0.9650 & 1.3757 & 1.3774 \\
\hline & 1.4462 & 1.4464 & 1.6679 & 1.6613 & 1.5470 & 1.5417 \\
\hline \multirow[t]{2}{*}{$3^{\prime}$} & 1.4647 & 1.4638 & 1.7295 & 1.7182 & 1.7233 & 1.7117 \\
\hline & 2.0982 & 2.0988 & 1.8979 & 1.8949 & 1.9216 & 1.9185 \\
\hline $6^{\prime}$ & 3.6824 & 3.7004 & 3.3849 & 3.3878 & 3.3778 & 3.3796 \\
\hline $8^{\prime}$ & 4.9515 & 4.9535 & 4.9034 & 4.9121 & 4.8998 & 4.9079 \\
\hline \multirow[t]{2}{*}{$9^{\prime}$} & 1.1929 & 1.2001 & 1.2558 & 1.2497 & 1.1522 & 1.1450 \\
\hline & 1.8561 & 1.8470 & 2.8658 & 2.8754 & 2.1775 & 2.1896 \\
\hline \multirow[t]{2}{*}{$13^{\prime}$} & 2.0246 & 2.0350 & 2.0553 & 2.0442 & 2.0682 & 2.0571 \\
\hline & 2.9042 & 2.8842 & 2.9065 & 2.9305 & 2.8972 & 2.9208 \\
\hline \multirow[t]{3}{*}{$14^{\prime}$} & 1.7568 & 1.7921 & 1.5363 & 1.5118 & 1.4009 & 1.3800 \\
\hline & 0.6366 & 0.6261 & 1.4210 & 1.4292 & 1.4351 & 1.4439 \\
\hline & 2.0574 & 2.0839 & 1.0228 & 1.0023 & 1.0287 & 1.0068 \\
\hline \multirow[t]{3}{*}{$15^{\prime}$} & 1.4404 & 1.4272 & 1.2890 & 1.2653 & 1.2948 & 1.1717 \\
\hline & 1.0594 & 1.0506 & 1.3041 & 1.3051 & 1.3162 & 1.2700 \\
\hline & 1.0031 & 1.0739 & 1.2816 & 1.2056 & 1.2454 & 1.3172 \\
\hline
\end{tabular}


Table S13. Calculated ${ }^{13} \mathrm{C}\left\{{ }^{1} \mathrm{H}\right\}$-NMR chemical shifts for conformers of $1 \mathrm{a}$ and $\mathbf{1 b}$.

\begin{tabular}{|c|c|c|c|c|c|c|}
\hline No. & & $\mathbf{a}$ & & & & \\
\hline & C1 & $\mathrm{C2}$ & C1 & $\mathrm{C2}$ & C3 & $\mathrm{C4}$ \\
\hline 1 & 57.1513 & 62.1627 & 62.1986 & 57.1457 & 62.1704 & 57.1394 \\
\hline 2 & 35.7453 & 36.0937 & 36.1281 & 35.7437 & 36.1093 & 35.7283 \\
\hline 3 & 124.8141 & 124.7041 & 124.2829 & 124.4045 & 124.3647 & 124.4828 \\
\hline 4 & 145.4351 & 145.5575 & 145.8993 & 145.7396 & 145.8436 & 145.6842 \\
\hline 5 & 53.7751 & 53.5279 & 53.537 & 53.854 & 53.531 & 53.8421 \\
\hline 6 & 32.2259 & 31.7813 & 31.9519 & 32.3472 & 31.929 & 32.3208 \\
\hline 7 & 168.2462 & 166.0339 & 164.5688 & 166.8259 & 164.6221 & 166.863 \\
\hline 8 & 107.8243 & 107.7954 & 107.4899 & 107.4733 & 107.4985 & 107.4805 \\
\hline 9 & 40.5424 & 39.3844 & 39.7641 & 40.896 & 39.7073 & 40.8534 \\
\hline 10 & 74.1887 & 74.3905 & 74.3528 & 74.1511 & 74.3384 & 74.139 \\
\hline 11 & 123.7639 & 124.6618 & 125.3495 & 124.4803 & 125.2713 & 124.412 \\
\hline 12 & 168.5775 & 168.3728 & 167.9484 & 168.136 & 167.89 & 168.0783 \\
\hline 13 & 24.6556 & 24.5755 & 24.7318 & 24.7811 & 24.6666 & 24.7138 \\
\hline 14 & 17.9659 & 17.9997 & 17.9947 & 17.9772 & 17.983 & 17.9656 \\
\hline 15 & 34.0237 & 33.9261 & 34.041 & 34.133 & 34.0112 & 34.1084 \\
\hline 1 ' & 76.0834 & 76.1298 & 73.0721 & 73.0156 & 74.1046 & 74.0525 \\
\hline 2 ' & 26.7797 & 26.7849 & 29.6748 & 29.6063 & 26.1656 & 26.1246 \\
\hline 3 & 28.2326 & 28.2546 & 31.7369 & 31.6795 & 31.5392 & 31.4828 \\
\hline $4 '$ & 67.9438 & 68.0079 & 66.0705 & 66.04 & 66.2481 & 66.2156 \\
\hline 5 & 71.8383 & 71.9473 & 73.0321 & 72.9794 & 72.9298 & 72.8805 \\
\hline 6 & 67.1544 & 66.7789 & 70.8632 & 71.1 & 70.7215 & 70.9495 \\
\hline 7 ' & 163.7096 & 164.1194 & 164.1427 & 163.7966 & 163.9423 & 163.6041 \\
\hline 8 & 76.3311 & 76.4833 & 76.8403 & 76.6574 & 76.4634 & 76.2776 \\
\hline 9' & 42.5057 & 42.5178 & 42.0524 & 42.1232 & 41.4036 & 41.4743 \\
\hline $10^{\prime}$ & 42.4817 & 42.495 & 43.7845 & 43.7456 & 43.8304 & 43.7873 \\
\hline $11^{\prime}$ & 128.4782 & 128.4858 & 129.0182 & 129.0831 & 129.0458 & 129.0977 \\
\hline $12^{\prime}$ & 167.9993 & 168.1576 & 168.2836 & 168.0939 & 168.0989 & 167.914 \\
\hline $13^{\prime}$ & 27.7279 & 27.8219 & 27.7021 & 27.611 & 27.6931 & 27.603 \\
\hline $14^{\prime}$ & 22.2344 & 22.1742 & 15.7489 & 15.7565 & 16.2947 & 16.3009 \\
\hline $15^{\prime}$ & 23.6756 & 23.7097 & 21.9469 & 21.9097 & 22.039 & 22.0014 \\
\hline
\end{tabular}




\section{ECD simulation:}

ECD spectrum of each conformation is simulated according to the overlapping Gaussian functions expressed as:

$$
\Delta \varepsilon(E)=\frac{1}{2.296 \times 10^{-39} \sqrt{\pi} \sigma} \sum_{i}^{\mathrm{A}} \Delta E_{i} \mathrm{R}_{i} e^{\left[-(E-\Delta E i)^{2} / \sigma^{2}\right]}
$$

Where $\sigma$ is half the bandwidth at 1/e peak height and expressed in energy units. The parameters $\Delta E_{i}$ and $R_{i}$ are the excitation energies and rotational strengths for the transition $i$, respectively.

The above function is converted to $\Delta \varepsilon, \lambda$ (wavelength) correlations as:

$$
\Delta \varepsilon(\lambda)=\frac{1}{2.296 \times 10^{-39} \sqrt{\pi} \sigma} \sum_{i}^{\mathrm{A}} \Delta E_{i} \mathrm{R}_{i} e^{\left[-(1240 / \lambda-\Delta E i)^{2} / \sigma^{2}\right]}
$$

and then simulation were accomplished by using the Excel 2003 and the Origin 7.0 software.

To get the final spectra, all the simulated spectra of conformations of each compound were averaged according to their energy and the Boltzmann distribution theory expressed as:

$$
\frac{N_{i}^{*}}{N}=\frac{g_{i} e^{-\varepsilon_{i} / k_{B} T}}{\sum g_{i} e^{-\varepsilon_{i} / k_{B} T}}
$$

Table S14. Calculated ECD Data for $1 \mathrm{~b}$ in gas phase.

\begin{tabular}{|l|l|l|l|l|l|l|l|l|}
\hline \multirow{2}{*}{ State } & \multicolumn{2}{|c|}{ C1 } & \multicolumn{2}{c|}{ C2 } & \multicolumn{2}{c|}{ C3 } \\
\hline \multirow{2}{*}{} & $\begin{array}{l}\text { Excitation } \\
\text { energies(eV) }\end{array}$ & $\begin{array}{l}\text { Rotatory } \\
\text { Strengths* }\end{array}$ & $\begin{array}{l}\text { Excitation } \\
\text { energies(eV) }\end{array}$ & $\begin{array}{l}\text { Rotatory } \\
\text { Strengths* }\end{array}$ & $\begin{array}{l}\text { Excitation } \\
\text { energies(eV) }\end{array}$ & $\begin{array}{l}\text { Rotatory } \\
\text { Strength* }\end{array}$ & $\begin{array}{l}\text { Excitation } \\
\text { energies(eV) }\end{array}$ & $\begin{array}{l}\text { Rotatory } \\
\text { Strengths* }\end{array}$ \\
\hline 1 & 4.9161 & -2.8888 & 4.917 & -3.844 & 4.9122 & -2.6658 & 4.9135 & -3.4501 \\
\hline 2 & 4.9364 & 3.393 & 4.9291 & 3.2078 & 4.9341 & 4.3835 & 4.9273 & 4.1774 \\
\hline 3 & 5.4838 & -87.088 & 5.3676 & -41.5736 & 5.4812 & -85.2342 & 5.3666 & -40.4368 \\
\hline 4 & 5.8551 & 190.1174 & 5.7511 & 47.8771 & 5.8477 & 175.8213 & 5.7524 & 44.016 \\
\hline 5 & 5.947 & 25.413 & 5.9295 & 61.0943 & 5.9043 & 4.5872 & 5.8876 & -9.9062 \\
\hline 6 & 6.0182 & -19.831 & 5.953 & 83.4094 & 6.0163 & 2.3873 & 5.9374 & 134.3066 \\
\hline 7 & 6.1348 & 9.9891 & 6.042 & -32.8616 & 6.1354 & 8.2713 & 6.0522 & -21.2686 \\
\hline 8 & 6.3518 & -24.818 & 6.3277 & -5.3211 & 6.3368 & -7.3086 & 6.3182 & 2.5666 \\
\hline
\end{tabular}




\begin{tabular}{|c|c|c|c|c|c|c|c|c|}
\hline 9 & 6.4564 & -51.4689 & 6.4294 & -66.4241 & 6.4528 & 10.6877 & 6.4002 & -4.11 \\
\hline 10 & 6.5021 & 15.7472 & 6.4534 & -2.2687 & 6.4718 & -54.101 & 6.442 & -66.7585 \\
\hline 11 & 6.5872 & -31.272 & 6.556 & -8.0382 & 6.5303 & -35.0488 & 6.4989 & -8.1995 \\
\hline 12 & 6.7544 & -9.7503 & 6.6471 & -12.8922 & 6.7302 & -5.9174 & 6.6397 & -16.2672 \\
\hline 13 & 6.8103 & 7.8554 & 6.7484 & -13.9157 & 6.8114 & 7.3359 & 6.7251 & -8.6903 \\
\hline 14 & 6.8848 & -41.2419 & 6.8505 & -17.4286 & 6.8793 & -44.3862 & 6.8488 & -18.2485 \\
\hline 15 & 6.9683 & 22.8879 & 6.9646 & 28.6499 & 6.9303 & 25.8512 & 6.9342 & 35.155 \\
\hline 16 & 7.0554 & 65.8484 & 7.0687 & 37.3518 & 7.0428 & 63.4952 & 7.0586 & 45.0517 \\
\hline 17 & 7.1084 & 15.3975 & 7.0991 & 51.1132 & 7.1063 & 17.5248 & 7.0999 & 9.5667 \\
\hline 18 & 7.1537 & 0.4851 & 7.1079 & -27.05 & 7.1784 & 2.2546 & 7.1309 & -1.8019 \\
\hline 19 & 7.246 & -0.0517 & 7.1875 & -8.6663 & 7.232 & -4.8592 & 7.1888 & -4.6907 \\
\hline 20 & 7.2695 & -1.5702 & 7.3351 & -5.8158 & 7.2754 & -0.2724 & 7.2846 & -3.2864 \\
\hline 21 & 7.3378 & -2.6955 & 7.3735 & -33.4404 & 7.2905 & -3.1611 & 7.3095 & -3.3863 \\
\hline 22 & 7.361 & -39.8335 & 7.4075 & 11.135 & 7.3129 & 10.1968 & 7.387 & 11.3835 \\
\hline 23 & 7.3755 & 6.4716 & 7.456 & -0.3527 & 7.3312 & -0.6621 & 7.4198 & -1.4757 \\
\hline 24 & 7.4238 & 21.1003 & 7.5062 & 10.8972 & 7.4128 & 19.0247 & 7.4838 & 7.4097 \\
\hline 25 & 7.4666 & -2.2818 & 7.5398 & 23.5236 & 7.4475 & -15.2954 & 7.5267 & 11.7579 \\
\hline 26 & 7.5842 & -8.4685 & 7.5921 & -0.0405 & 7.5192 & 11.2565 & 7.5329 & 20.2452 \\
\hline 27 & 7.6015 & -2.2029 & 7.5954 & -4.6586 & 7.5851 & -9.1485 & 7.6037 & -6.2832 \\
\hline 28 & 7.6733 & -5.6171 & 7.6295 & 14.2305 & 7.6688 & -7.0187 & 7.6113 & 0.38 \\
\hline 29 & 7.6902 & -0.909 & 7.6476 & -3.6502 & 7.7 & -3.8649 & 7.642 & 6.9426 \\
\hline 30 & 7.7158 & 10.7448 & 7.6763 & -7.7694 & 7.7137 & 9.4416 & 7.6559 & -13.0737 \\
\hline 31 & 7.7262 & 13.1773 & 7.7177 & 0.4915 & 7.726 & 4.4134 & 7.6821 & -7.8632 \\
\hline 32 & 7.7314 & -0.382 & 7.7357 & -16.5913 & 7.735 & 6.3096 & 7.7476 & -3.9137 \\
\hline 33 & 7.7836 & -3.3899 & 7.8364 & 9.4008 & 7.7803 & -4.9286 & 7.8242 & -11.7913 \\
\hline 34 & 7.8453 & 10.1255 & 7.8422 & -1.0476 & 7.8376 & 5.0764 & 7.8334 & 8.9548 \\
\hline 35 & 7.8906 & 2.9909 & 7.8707 & -8.0488 & 7.857 & -4.4538 & 7.8706 & 1.0702 \\
\hline 36 & 7.9213 & -9.2428 & 7.8972 & 2.16 & 7.8816 & 0.2904 & 7.8919 & -1.4293 \\
\hline 37 & 7.9497 & -0.5507 & 7.938 & 11.5785 & 7.9201 & 10.8475 & 7.9352 & 29.5849 \\
\hline 38 & 7.9934 & 2.7447 & 7.951 & 1.4917 & 7.9952 & 2.4154 & 7.9596 & -1.3799 \\
\hline 39 & 8.0563 & 0.7287 & 8.0309 & -0.8244 & 8.0309 & 4.2074 & 8.0289 & 0.2966 \\
\hline 40 & 8.0903 & 26.1109 & 8.0509 & 2.0903 & 8.088 & 10.8244 & 8.0629 & 3.3339 \\
\hline 41 & 8.1046 & -2.872 & 8.0726 & 12.5183 & 8.0937 & 9.2904 & 8.0982 & 10.5337 \\
\hline 42 & 8.1676 & 9.1556 & 8.1096 & -7.9417 & 8.1664 & 7.198 & 8.1077 & -7.4576 \\
\hline 43 & 8.1818 & 7.7364 & 8.2005 & -2.3798 & 8.182 & 19.8101 & 8.2043 & 0.5359 \\
\hline 44 & 8.1997 & 3.1542 & 8.2073 & 6.8323 & 8.2408 & 0.6644 & 8.2165 & 11.6812 \\
\hline 45 & 8.2489 & -16.679 & 8.2556 & -2.151 & 8.2462 & -13.4386 & 8.2782 & -4.4093 \\
\hline 46 & 8.2616 & -6.2761 & 8.299 & -17.3755 & 8.2854 & 20.0354 & 8.2982 & 1.825 \\
\hline 47 & 8.2929 & -8.5779 & 8.3061 & -10.5379 & 8.299 & -4.9313 & 8.314 & -35.6493 \\
\hline 48 & 8.3029 & 19.2192 & 8.331 & -17.632 & 8.3137 & -1.9423 & 8.3424 & -39.2774 \\
\hline 49 & 8.3398 & -20.8344 & 8.3509 & -51.4754 & 8.3521 & -23.2224 & 8.3549 & -0.0125 \\
\hline 50 & 8.3521 & 10.7797 & 8.3723 & 4.8746 & 8.3612 & 5.8569 & 8.3748 & -7.2377 \\
\hline 51 & 8.3786 & 5.2339 & 8.3918 & 31.4758 & 8.3904 & 10.2664 & 8.3934 & 50.8032 \\
\hline
\end{tabular}




\begin{tabular}{|l|l|l|l|l|l|l|l|l|}
\hline 52 & 8.4149 & -0.9597 & 8.3984 & 9.1489 & 8.4108 & -20.9884 & 8.4008 & -1.4678 \\
\hline 53 & 8.4505 & -3.942 & 8.423 & 10.2721 & 8.4471 & -10.0422 & 8.4194 & -10.479 \\
\hline 54 & 8.4578 & -57.1248 & 8.4617 & -37.5983 & 8.4711 & -46.3453 & 8.4334 & -21.0747 \\
\hline 55 & 8.4681 & 0.892 & 8.4724 & -11.7295 & 8.4987 & 17.1118 & 8.4704 & -34.3717 \\
\hline 56 & 8.4868 & -0.703 & 8.4794 & 5.9278 & 8.5058 & -6.5437 & 8.4896 & 21.8169 \\
\hline 57 & 8.4985 & 5.9832 & 8.4896 & 12.506 & 8.5164 & -17.3924 & 8.5077 & -8.6564 \\
\hline 58 & 8.5076 & -8.2091 & 8.5181 & -2.7193 & 8.539 & -12.5295 & 8.5279 & -3.2087 \\
\hline 59 & 8.5186 & -7.7478 & 8.5324 & 8.2547 & 8.5466 & -16.2731 & 8.5533 & -9.961 \\
\hline 60 & 8.5593 & 1.5958 & 8.5342 & -1.379 & 8.5774 & -51.2004 & 8.5671 & -26.035 \\
\hline
\end{tabular}

* $\mathrm{R}$ (velocity) 10**-40 erg-esu-cm

Table S15. Calculated ECD Data for 2 in gas phase.

\begin{tabular}{|c|c|c|c|c|c|c|}
\hline \multirow[b]{2}{*}{ State } & \multicolumn{2}{|c|}{ C1 } & \multicolumn{2}{|c|}{$\mathrm{C2}$} & \multicolumn{2}{|c|}{$\mathrm{C3}$} \\
\hline & $\begin{array}{l}\text { Excitation } \\
\text { energies }(\mathrm{eV})\end{array}$ & $\begin{array}{l}\text { Rotatory } \\
\text { Strengths* }\end{array}$ & $\begin{array}{l}\text { Excitation } \\
\text { energies }(\mathrm{eV})\end{array}$ & $\begin{array}{l}\text { Rotatory } \\
\text { Strengths* }\end{array}$ & $\begin{array}{l}\text { Excitation } \\
\text { energies }(\mathrm{eV})\end{array}$ & $\begin{array}{l}\text { Rotatory } \\
\text { Strengths* }\end{array}$ \\
\hline 1 & 4.9324 & 1.1981 & 4.9247 & 0.7261 & 4.8123 & -2.0343 \\
\hline 2 & 5.0147 & 4.3899 & 5.0155 & 4.5895 & 5.0105 & -3.4986 \\
\hline 3 & 5.0227 & -3.7309 & 5.0202 & -1.0489 & 5.0619 & -11.573 \\
\hline 4 & 5.5972 & -264.6622 & 5.5891 & -274.5578 & 5.5886 & -216.4144 \\
\hline 5 & 5.697 & 191.0543 & 5.6583 & 158.7281 & 5.66 & 132.6096 \\
\hline 6 & 5.8686 & 50.0833 & 5.8699 & 83.2997 & 5.8195 & 36.733 \\
\hline 7 & 6.0481 & 114.6104 & 6.0482 & 113.4588 & 6.0226 & 142.9503 \\
\hline 8 & 6.1876 & -81.9048 & 6.2038 & -84.1001 & 6.1311 & -117.2567 \\
\hline 9 & 6.3438 & -51.2215 & 6.3255 & -36.7802 & 6.231 & 12.1161 \\
\hline 10 & 6.4271 & -52.6129 & 6.4056 & -60.0251 & 6.2813 & -17.7403 \\
\hline 11 & 6.5228 & 1.86 & 6.5361 & 1.7946 & 6.4629 & -54.3582 \\
\hline 12 & 6.5926 & -26.6702 & 6.5871 & 9.5631 & 6.5892 & -24.2063 \\
\hline 13 & 6.6239 & -0.5122 & 6.5996 & -49.3229 & 6.6389 & -11.9105 \\
\hline 14 & 6.6829 & -12.602 & 6.6821 & -17.0731 & 6.6613 & 15.0617 \\
\hline 15 & 6.7811 & 2.8679 & 6.784 & 0.7109 & 6.7458 & 27.8985 \\
\hline 16 & 6.8347 & -16.3402 & 6.8345 & -6.2514 & 6.8139 & -2.5639 \\
\hline 17 & 6.9713 & 3.6521 & 6.9205 & 11.2709 & 6.887 & -0.6084 \\
\hline 18 & 7.0053 & 34.72 & 7.0241 & 12.1501 & 6.9689 & 6.8247 \\
\hline 19 & 7.0509 & 8.5586 & 7.0593 & 26.718 & 7.0019 & 10.4501 \\
\hline 20 & 7.0638 & -2.438 & 7.0772 & -0.5374 & 7.0513 & -33.8287 \\
\hline 21 & 7.1328 & 7.9211 & 7.1241 & 21.2411 & 7.0964 & 3.2383 \\
\hline 22 & 7.1707 & 9.8082 & 7.2203 & 8.6855 & 7.1335 & 7.6366 \\
\hline 23 & 7.2525 & -14.9885 & 7.2427 & -17.1081 & 7.2279 & -39.1044 \\
\hline 24 & 7.265 & 5.9656 & 7.2714 & 13.2165 & 7.2542 & 46.3372 \\
\hline 25 & 7.2914 & 6.9148 & 7.297 & 4.8486 & 7.2998 & 44.9774 \\
\hline
\end{tabular}




\begin{tabular}{|c|c|c|c|c|c|c|}
\hline 26 & 7.3435 & 40.5077 & 7.3516 & -12.4613 & 7.3652 & 37.6329 \\
\hline 27 & 7.3628 & 11.4066 & 7.373 & 83.3051 & 7.3874 & 24.853 \\
\hline 28 & 7.4009 & 32.2081 & 7.4056 & 13.1767 & 7.4007 & -7.3718 \\
\hline 29 & 7.4499 & 5.023 & 7.4662 & 35.1442 & 7.4206 & -13.7461 \\
\hline 30 & 7.4618 & -16.0549 & 7.483 & -4.4565 & 7.4538 & 0.1897 \\
\hline 31 & 7.477 & -11.3516 & 7.4981 & -19.3182 & 7.4652 & -4.8733 \\
\hline 32 & 7.4961 & -1.1928 & 7.5079 & -13.9787 & 7.4885 & -24.2299 \\
\hline 33 & 7.5088 & -26.9414 & 7.521 & -1.7153 & 7.5411 & 37.9551 \\
\hline 34 & 7.5331 & 26.3116 & 7.5359 & 17.0576 & 7.5632 & -10.5241 \\
\hline 35 & 7.5616 & 18.8837 & 7.5738 & -1.1392 & 7.6375 & 6.335 \\
\hline 36 & 7.6197 & -41.9919 & 7.63 & -21.167 & 7.6433 & 15.3068 \\
\hline 37 & 7.6556 & 14.5093 & 7.66 & 15.4047 & 7.6521 & -58.7896 \\
\hline 38 & 7.6811 & 0.6476 & 7.6691 & -14.71 & 7.6931 & -12.313 \\
\hline 39 & 7.7008 & -14.6921 & 7.697 & 1.9826 & 7.6973 & 6.0581 \\
\hline 40 & 7.7278 & -29.62 & 7.74 & -21.2386 & 7.7274 & 5.4741 \\
\hline 41 & 7.7692 & -6.8285 & 7.7821 & 3.0896 & 7.7389 & -15.7127 \\
\hline 42 & 7.7897 & 6.0208 & 7.7928 & -14.1966 & 7.7878 & 14.603 \\
\hline 43 & 7.8004 & -8.5948 & 7.8307 & -5.224 & 7.8138 & -33.0715 \\
\hline 44 & 7.8343 & -14.6047 & 7.8448 & 52.4164 & 7.8639 & 11.1747 \\
\hline 45 & 7.8472 & 33.4138 & 7.8556 & -3.3697 & 7.872 & -47.7881 \\
\hline 46 & 7.8784 & -2.5809 & 7.879 & 7.2702 & 7.8804 & 43.8409 \\
\hline 47 & 7.8936 & -3.1979 & 7.8904 & -6.1975 & 7.929 & 2.6287 \\
\hline 48 & 7.9012 & 0.6591 & 7.9244 & -1.442 & 7.9672 & 13.0706 \\
\hline 49 & 7.946 & 16.9304 & 7.9916 & -8.9841 & 7.969 & -3.8855 \\
\hline 50 & 7.9869 & 26.8225 & 7.9984 & -4.8364 & 7.9834 & -11.438 \\
\hline 51 & 8.0066 & -8.0175 & 8.0182 & -24.7134 & 7.9872 & -7.8809 \\
\hline 52 & 8.0124 & 4.7043 & 8.0278 & 12.8096 & 8.0003 & 37.7022 \\
\hline 53 & 8.0274 & 12.2977 & 8.0767 & 19.3758 & 8.0242 & 46.451 \\
\hline 54 & 8.082 & -33.8929 & 8.0865 & -80.7453 & 8.0315 & -13.0391 \\
\hline 55 & 8.1011 & 9.0192 & 8.1067 & 12.3551 & 8.0614 & 19.5703 \\
\hline 56 & 8.1325 & -13.2365 & 8.1298 & 22.9415 & 8.0735 & -29.2759 \\
\hline 57 & 8.1853 & 1.1207 & 8.1464 & -42.0731 & 8.1317 & -17.6696 \\
\hline 58 & 8.1888 & -7.1506 & 8.1787 & 11.0151 & 8.142 & -3.3613 \\
\hline 59 & 8.2142 & -6.3988 & 8.21 & -1.1844 & 8.1853 & -5.2165 \\
\hline 60 & 8.2238 & -18.7692 & 8.2299 & 0.1165 & 8.2161 & 10.6922 \\
\hline
\end{tabular}

* $\mathrm{R}$ (velocity) $10 * *-40$ erg-esu-cm 


\section{Calculated Specific OR Data for Compounds 1 and 2.}

In order to reinforce the above conclusions, specific optical rotations (OR) were further calculated for 1 and 2 at the M062X/TZVP//B3LYP/6-31G(d) level in gas phase. The theoretical specific OR value for $\mathbf{1}(+13.00)$ and the experimental data $(+15.26)$ were both positive. For compound 2, the theoretical OR value (-142.42) and experimental data $(-118.67)$ showed similar negative numbers. Hence, the results of ECD and specific OR calculations are in accordance with experimental ones, which all gave solid evidences for the absolute configurations of 1 as $1 R, 5 R, 8 S, 10 R, 1^{\prime} R, 4^{\prime} R, 5^{\prime} R, 6^{\prime} R, 8^{\prime} S$ and 2 as $1 R, 3 S, 5 S, 8 S, 9 S, 10 S, 1^{\prime} R, 6^{\prime} R, 8^{\prime} S, 10^{\prime} R$.

\begin{tabular}{|l|c|c|l|}
\hline \multicolumn{3}{|c|}{1} \\
\cline { 1 - 2 } Conf & Alpha[D](vacuum) & Averaged value & Experimental value \\
\hline C1 & 10.75 & & \\
\cline { 1 - 2 } C2 & 3.33 & \multirow{2}{*}{+12.99623926} & +15.26 \\
\cline { 1 - 2 } C3 & 32.12 & & \\
\hline C4 & 24.76 & & \\
\hline
\end{tabular}

\begin{tabular}{|l|l|l|l|}
\hline \multicolumn{4}{|c|}{2} \\
\hline Conf & Alpha[D](vacuum) & Averaged value & Experimental value \\
\hline C1 & -139.81 & & \\
C2 & -123.55 & -142.4246166 & -118.67 \\
\hline C3 & -146.02 & & \\
\hline
\end{tabular}


Figure S6. ${ }^{1} \mathrm{H}$ NMR spectrum $(500 \mathrm{MHz})$ of horienoid A (1) in methanol- $d_{4}$.

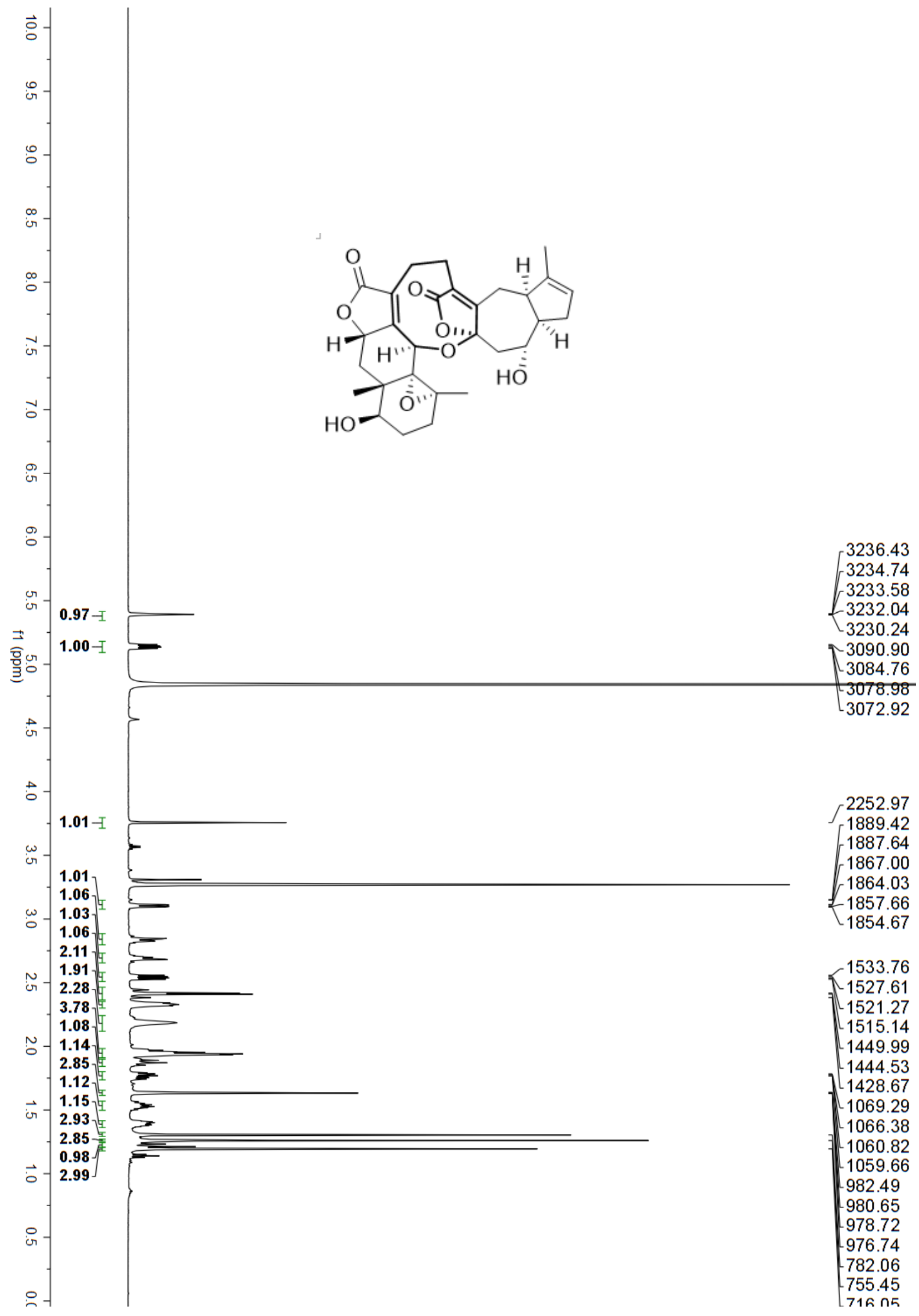


Figure S7. ${ }^{13} \mathrm{C}\left\{{ }^{1} \mathrm{H}\right\}$ NMR spectrum $(125 \mathrm{MHz})$ of horienoid A (1) in methanol- $d_{4}$.

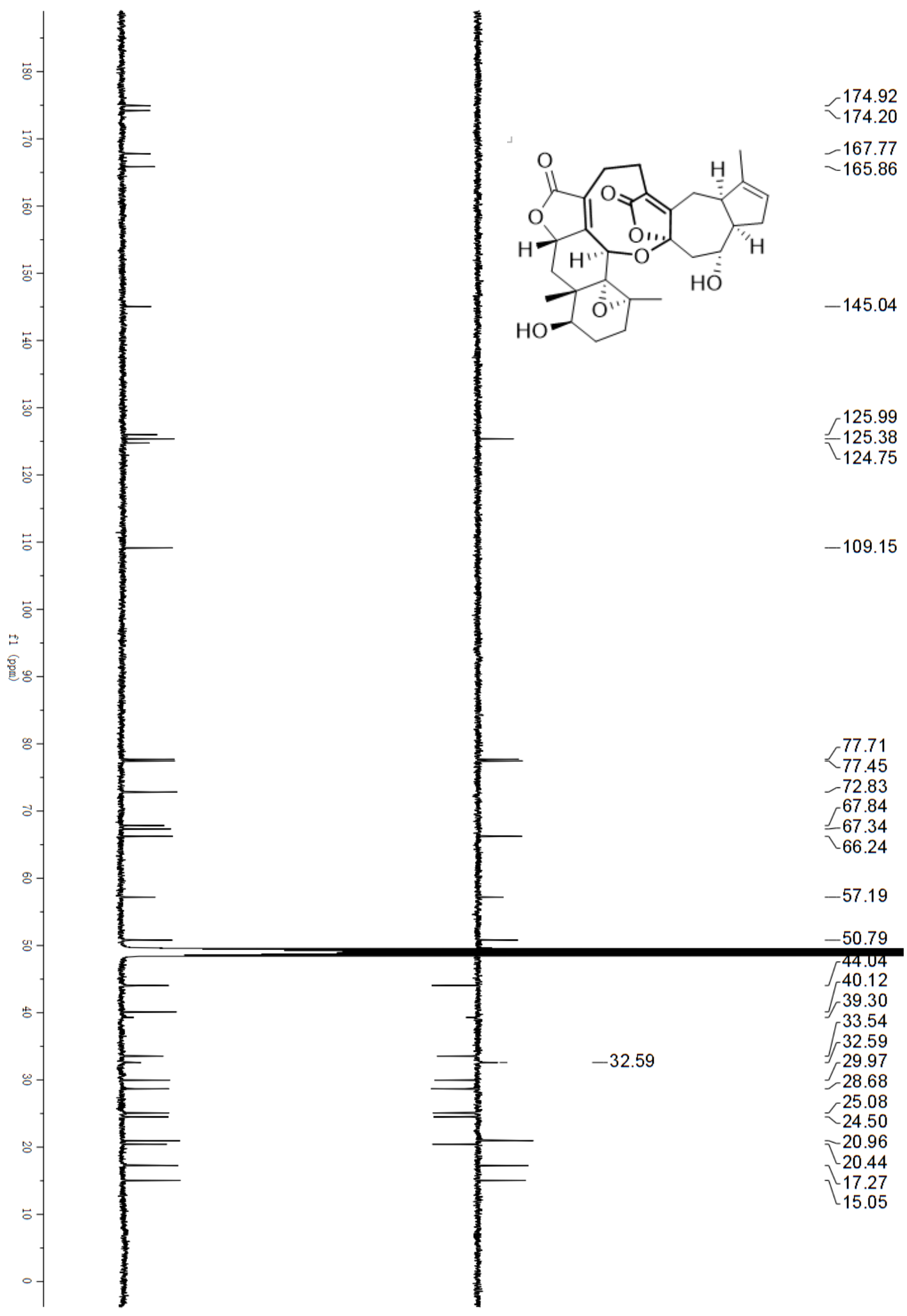


Figure S8. ${ }^{1} \mathrm{H}-{ }^{1} \mathrm{H}$ COSY spectrum $(500 \mathrm{MHz})$ of horienoid A (1) in methanol- $d 4$.

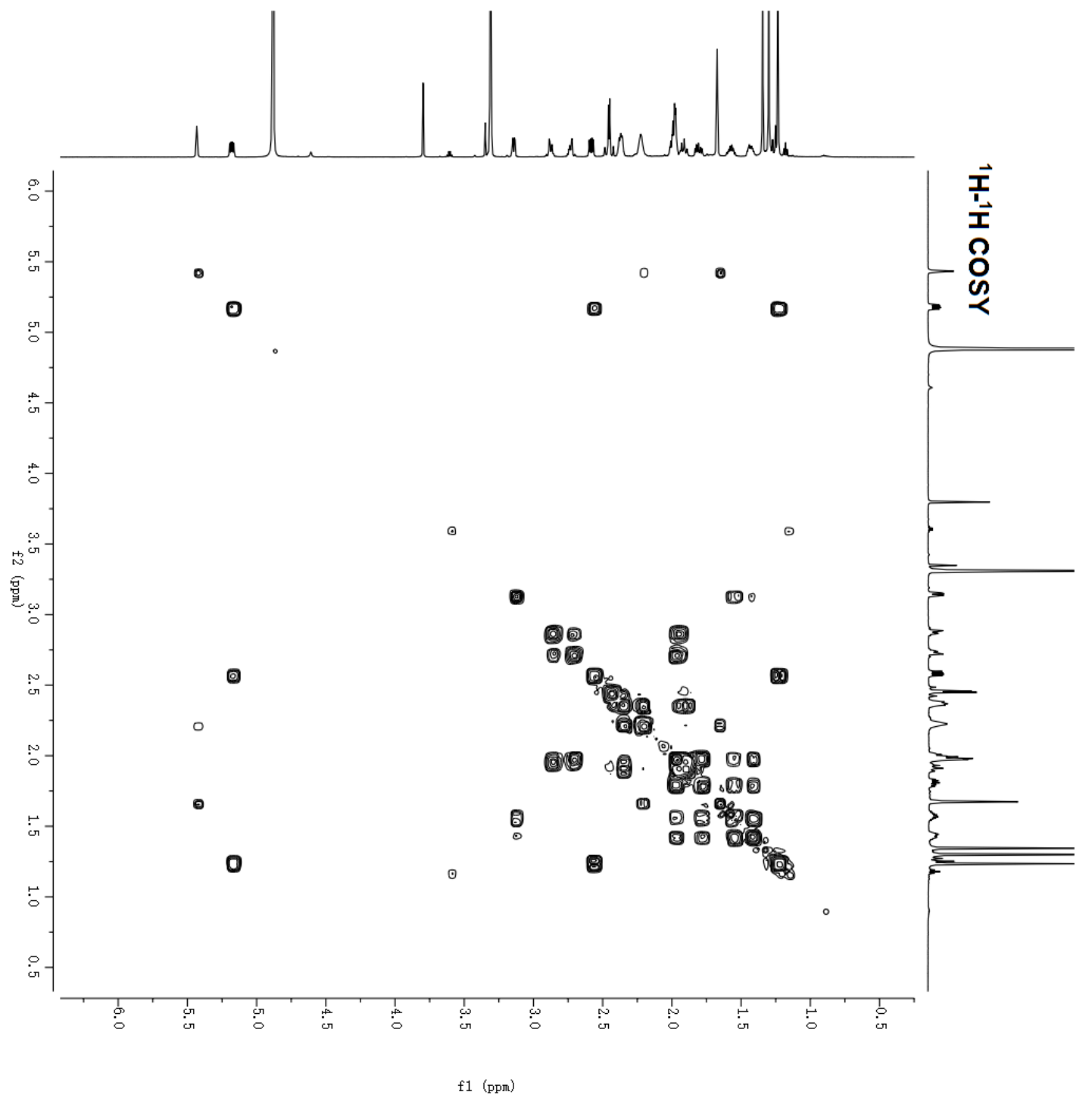


Figure S9. HSQC spectrum $(500 \mathrm{MHz})$ of horienoid A (1) in methanol- $d_{4}$.

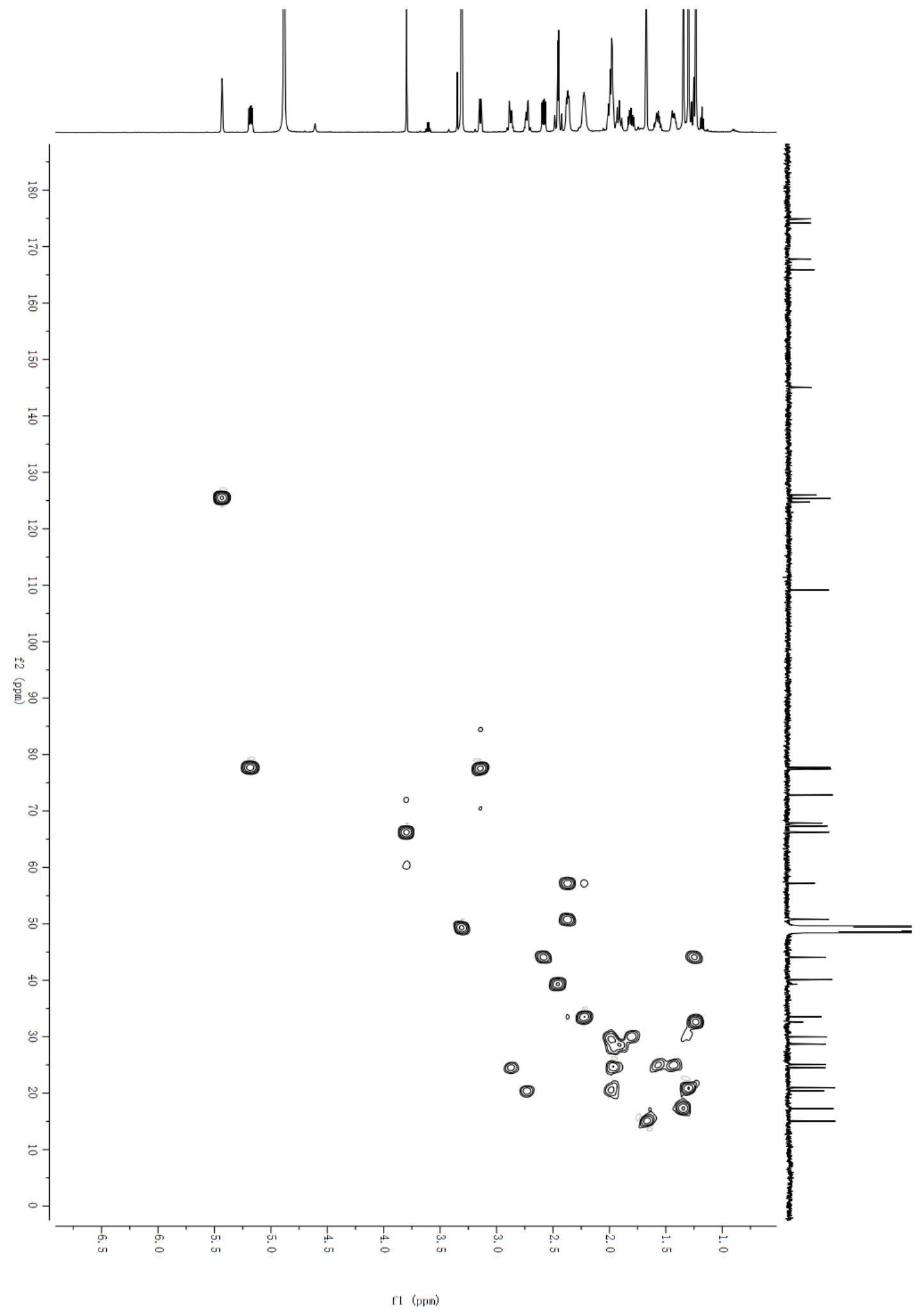


Figure S10. HMBC spectrum (500 MHz) of horienoid A (1) in methanol- $d_{4}$.

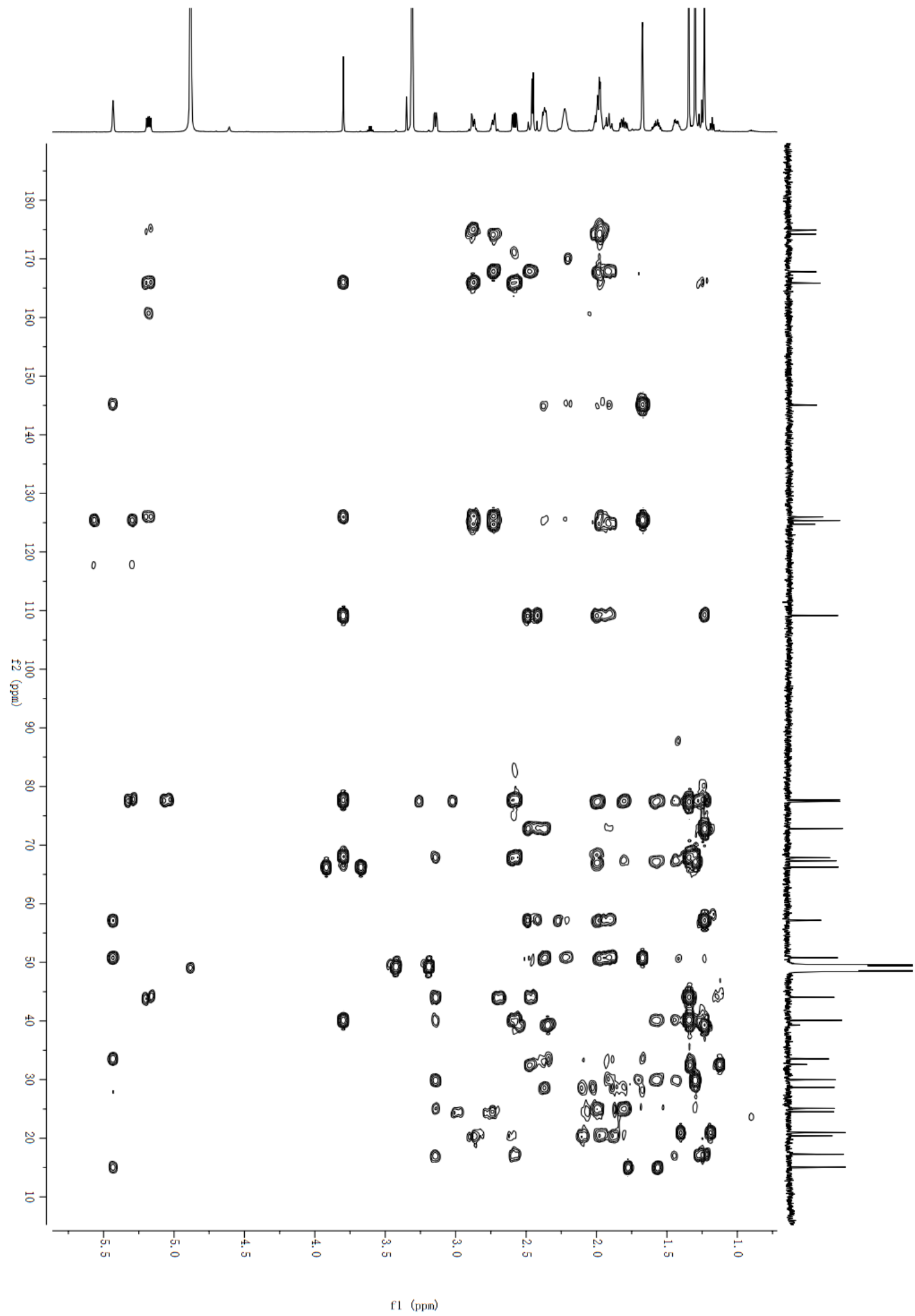


Figure S11. NOESY spectrum (500 MHz) of horienoid A (1) in methanol- $d_{4}$.

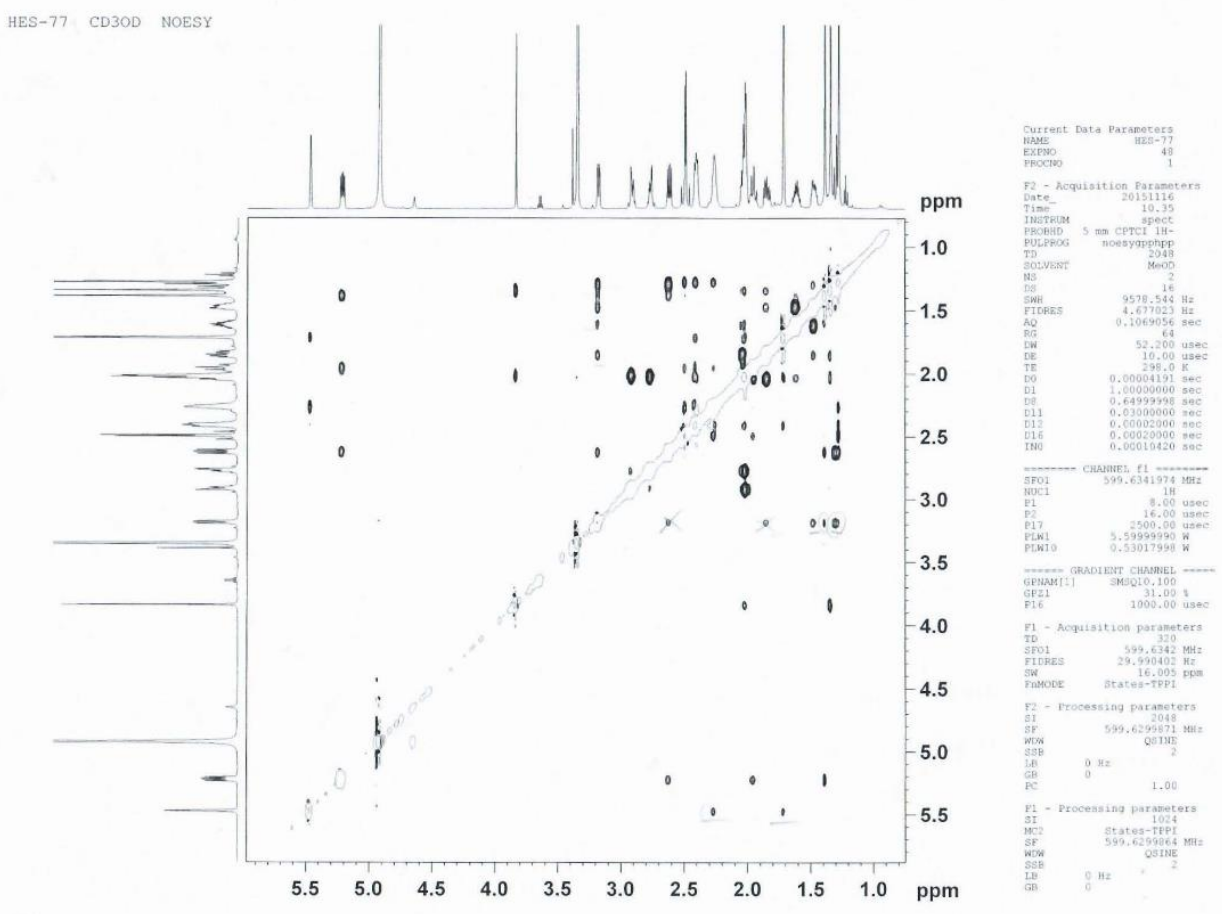


Figure S12. ${ }^{1} \mathrm{H}$ NMR spectrum $(500 \mathrm{MHz})$ of horienoid A (1) in pyridine- $d 5$.

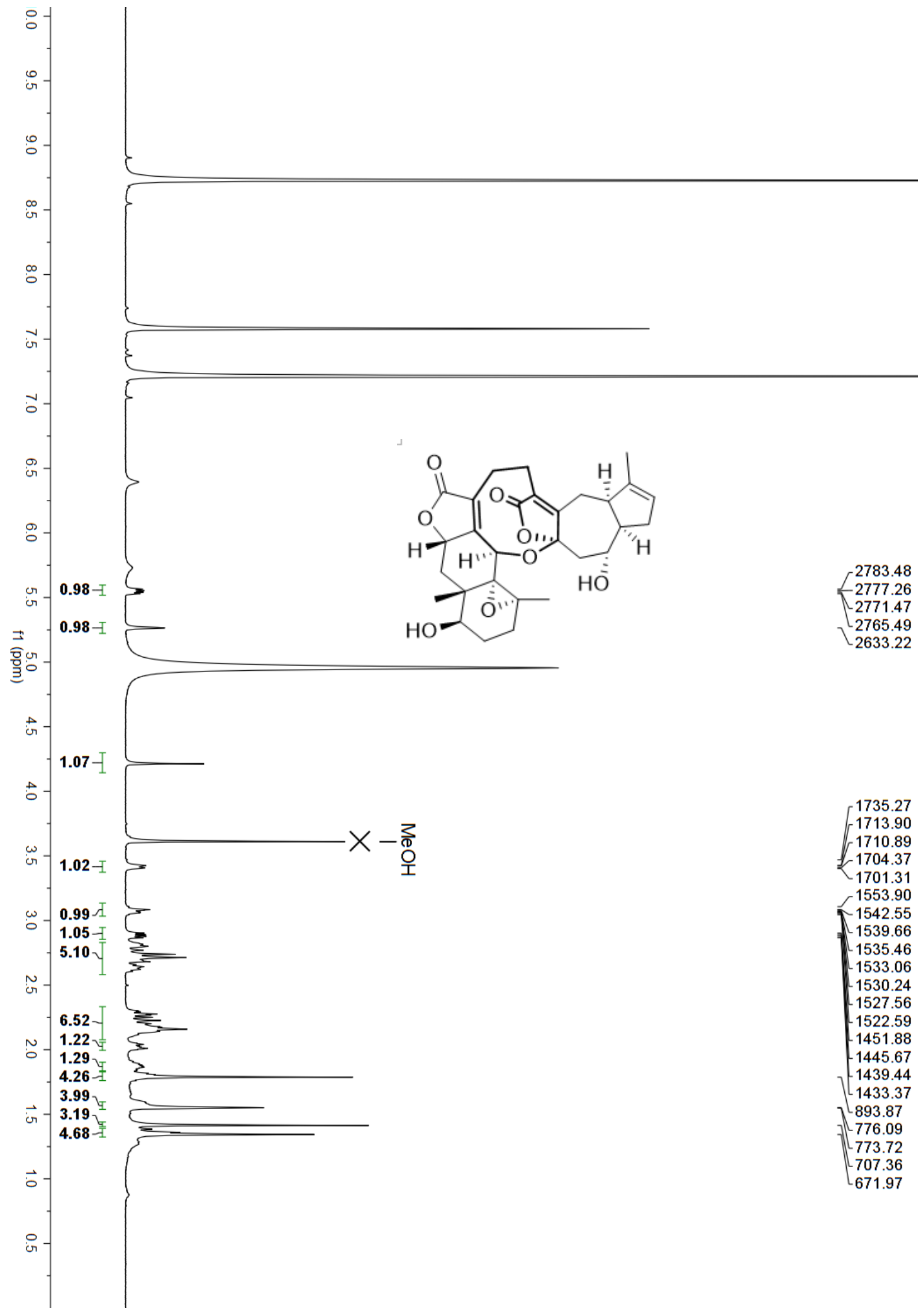


Figure S13. ${ }^{13} \mathrm{C}\left\{{ }^{1} \mathrm{H}\right\}$ NMR spectrum $(125 \mathrm{MHz})$ of horienoid A (1) in pyridine- $d 5$.

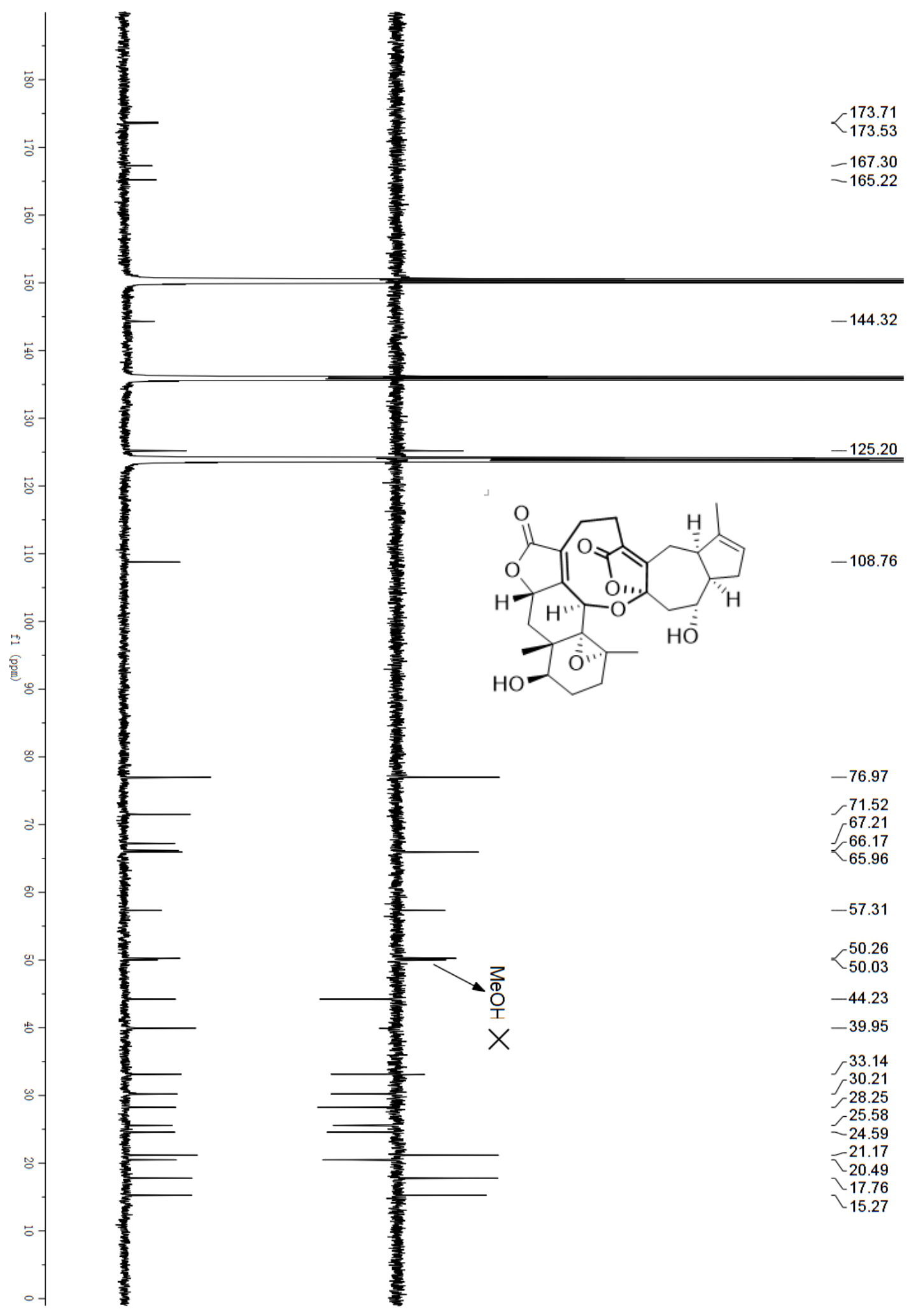


Figure S14. ${ }^{1} \mathrm{H}-{ }^{1} \mathrm{H}$ COSY spectrum $(500 \mathrm{MHz})$ of horienoid A (1) in pyridine- $d 5$.

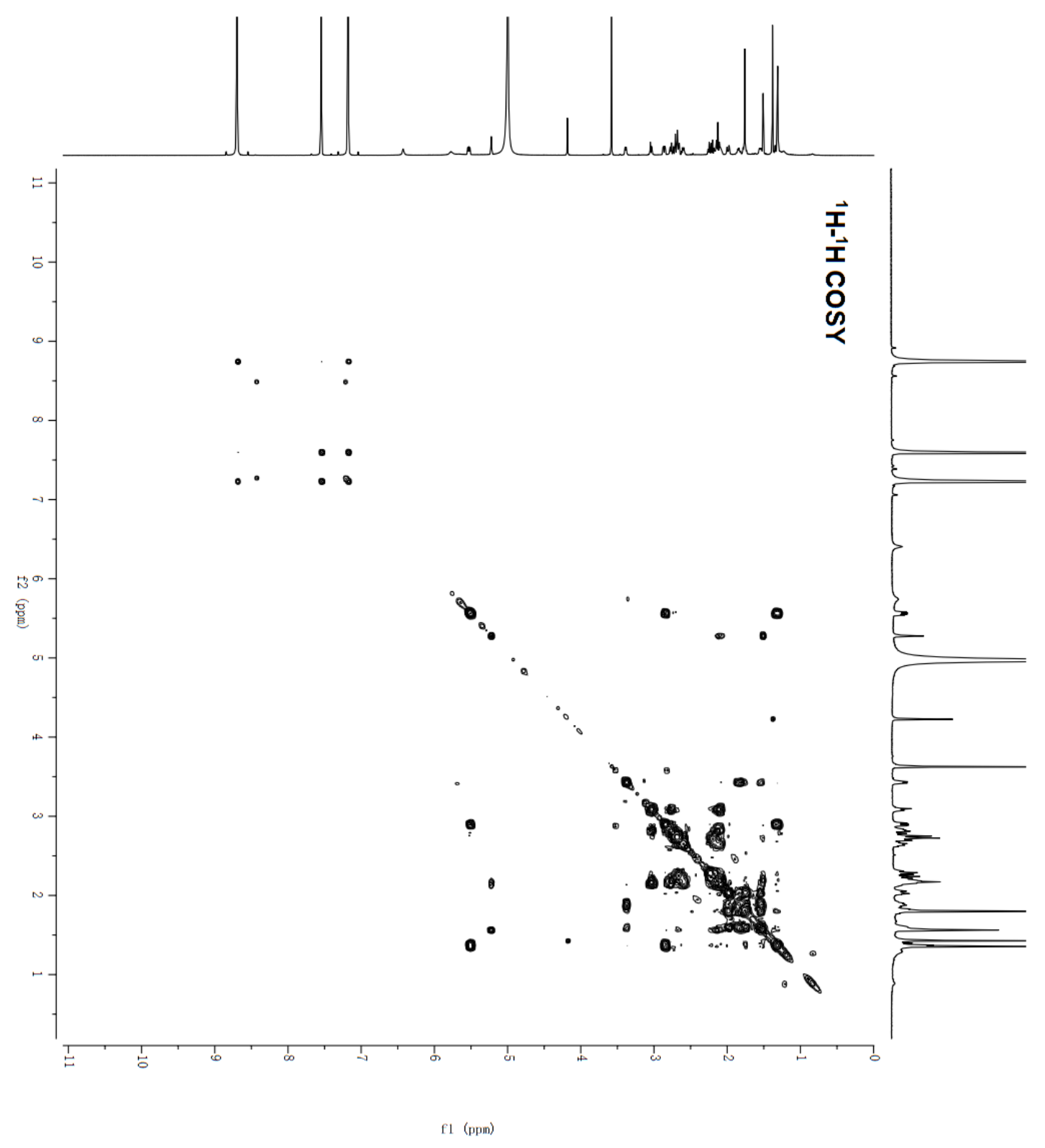


Figure S15. HSQC spectrum $(500 \mathrm{MHz})$ of horienoid A (1) in pyridine- $d 5$.

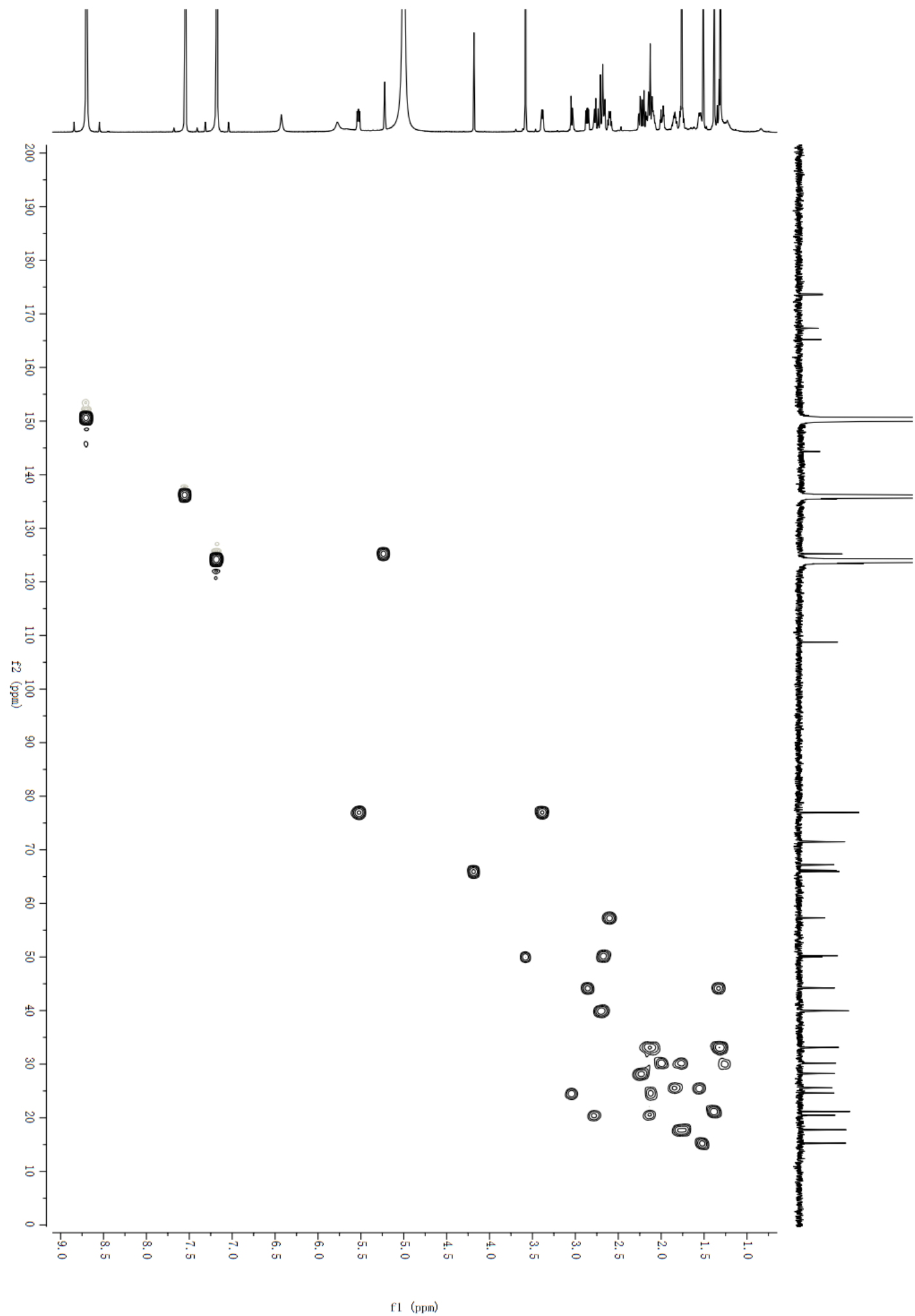


Figure S16. HMBC spectrum $(500 \mathrm{MHz})$ of horienoid A (1) in pyridine- $d_{5}$.

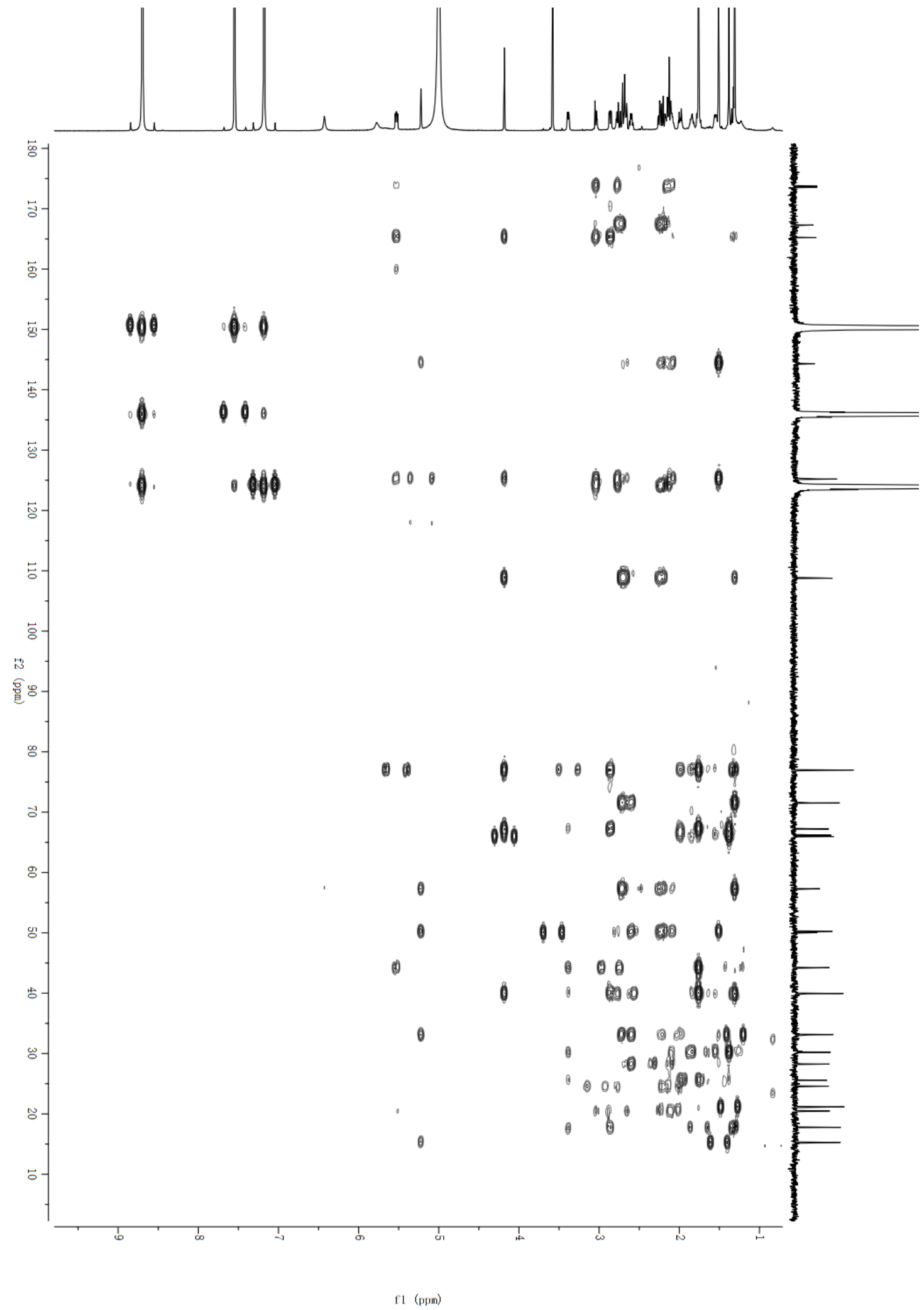


Figure S17. NOESY spectrum $(500 \mathrm{MHz})$ of horienoid A (1) in pyridine- $d_{5}$.

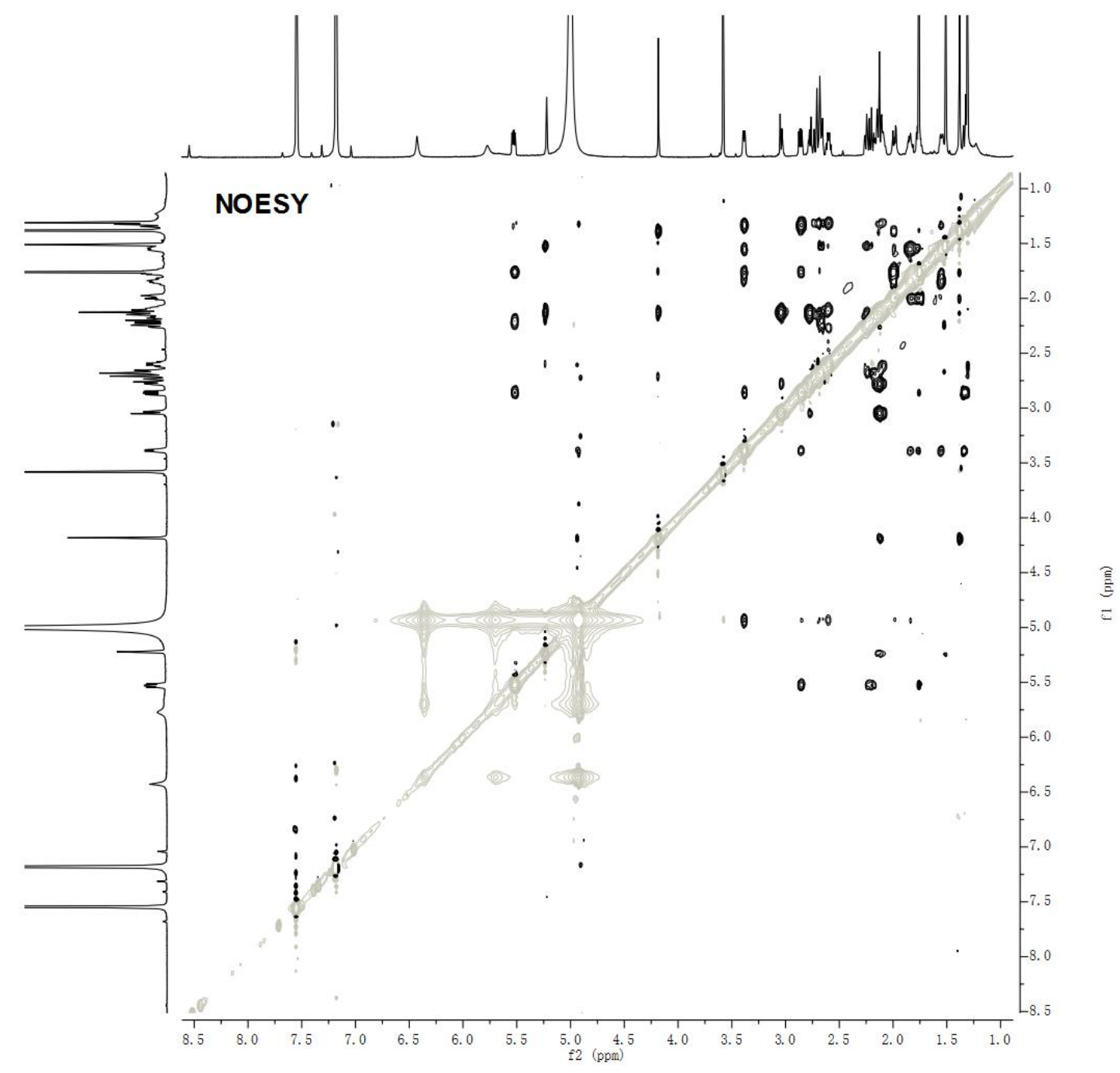


Figure S18. (+)-ESIMS spectrum of horienoid A (1).

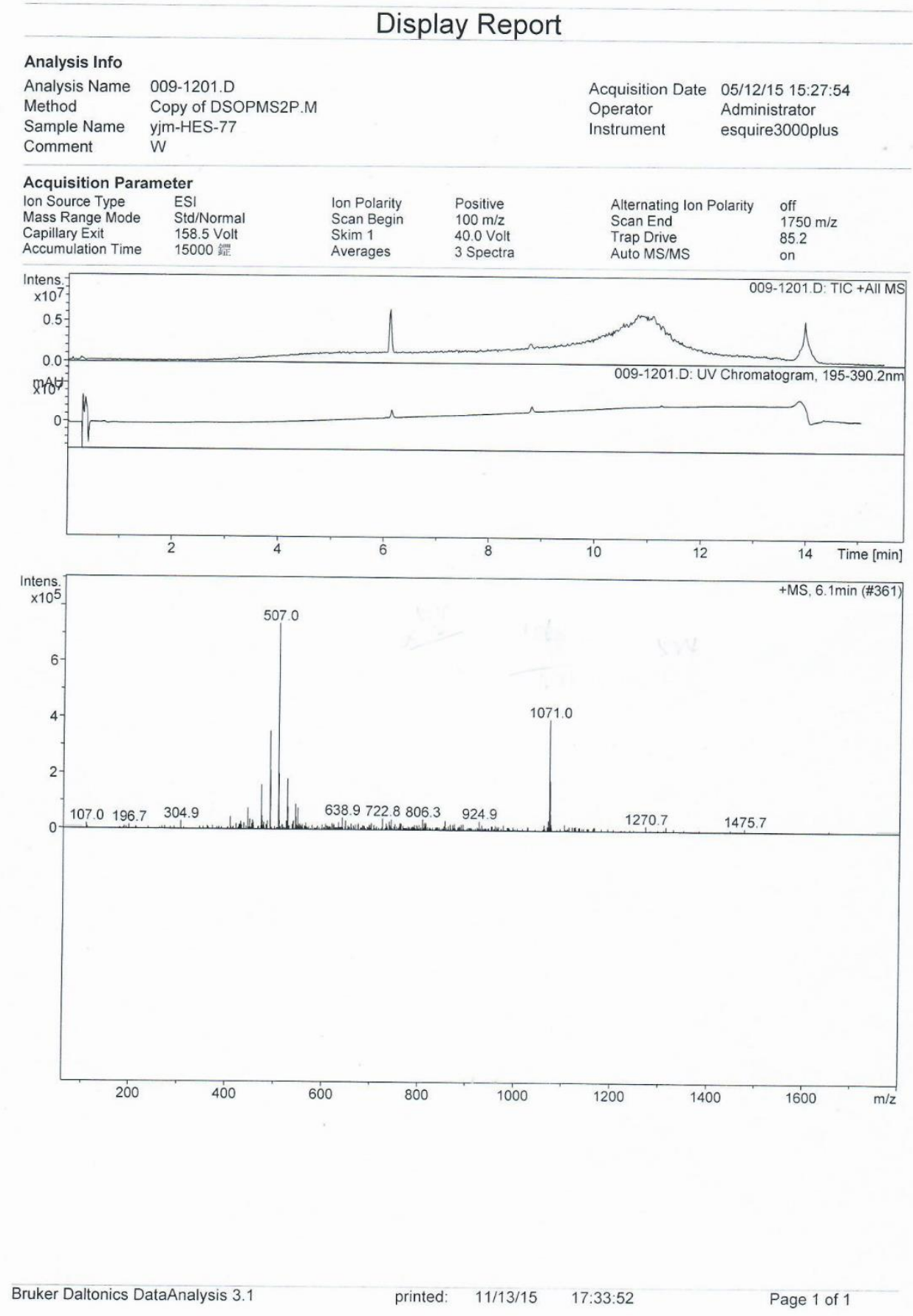


Figure S19. (-)-ESIMS spectrum of horienoid A (1).

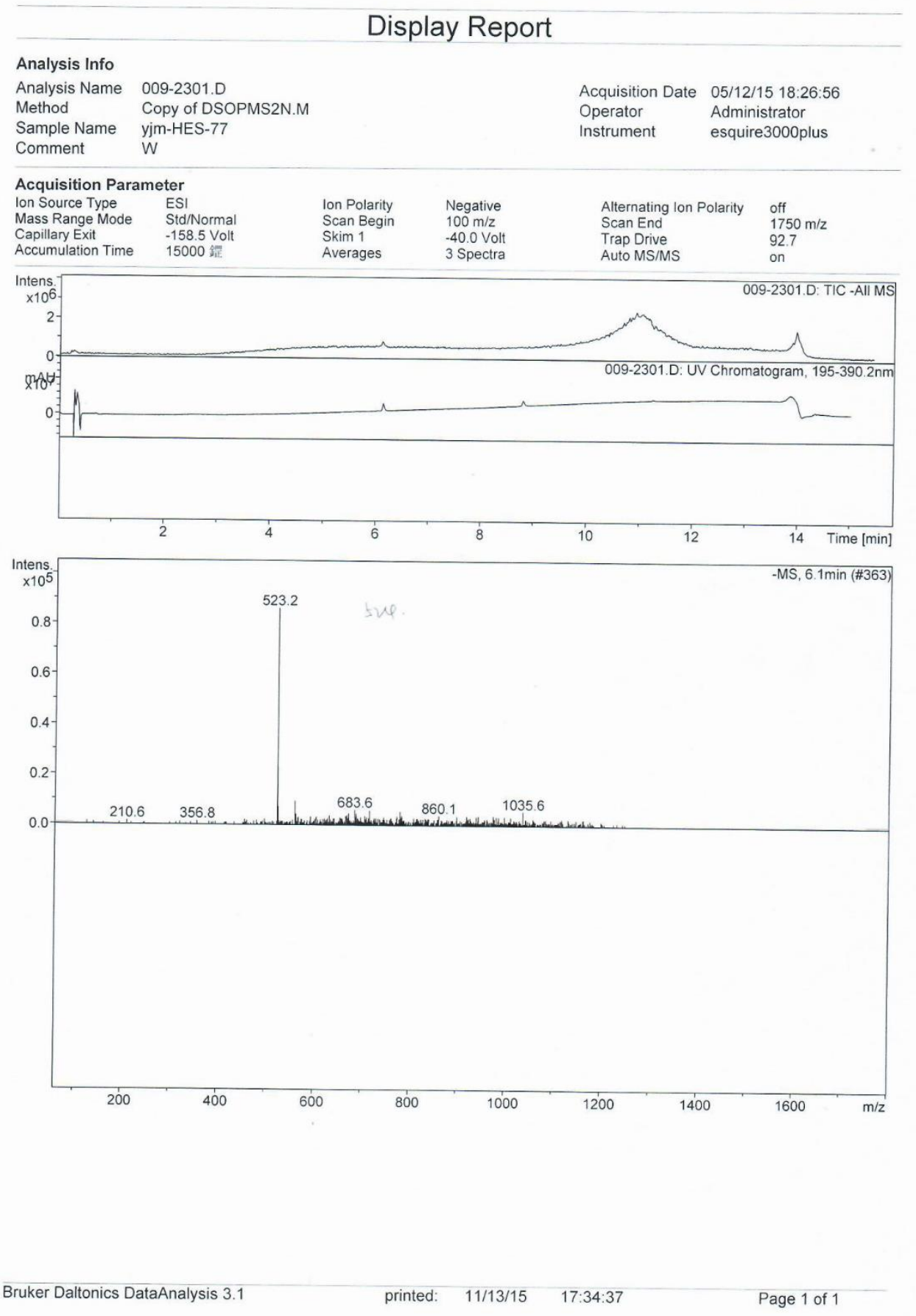


Figure S20. (-)-HRESIMS spectrum of horienoid A (1).

\section{Elemental Composition Report}

Single Mass Analysis

Tolerance $=3.0$ PPM / DBE: $\min =-1.5, \max =50.0$

Element prediction: Off

Number of isotope peaks used for $\mathrm{i}-\mathrm{FIT}=3$

Monoisotopic Mass, Even Electron Ions

205 formula(e) evaluated with 1 results within limits (up to 50 closest results for each mass)

Elements Used:

$\begin{array}{llll}\text { C: } 5-80 & \text { H: } 2-120 & \text { O: 0-20 } & \mathrm{Na}: 0-1\end{array}$

HES-77N

LCT PXE KE324

HES-77N_20151117 16 (0.335) AM2 (Ar, 10000.0,0.00,1.00); ABS; Cm (5:17)

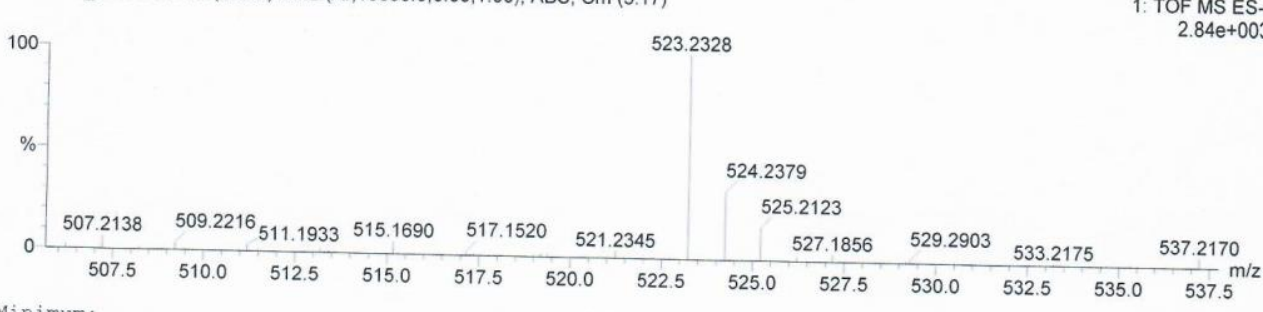

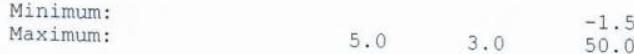

Mass Calc. Mass mDa

DBE i-FIT i-FIT (Norm) Formula

$\begin{array}{llllllllll}23.2328 & 523.2332 & -0.4 & -0.8 & 13.5 & 92.2 & 0.0 & \text { C30 } & \text { H35 } & 08\end{array}$ 
Figure S21. IR spectrum of horienoid A (1).

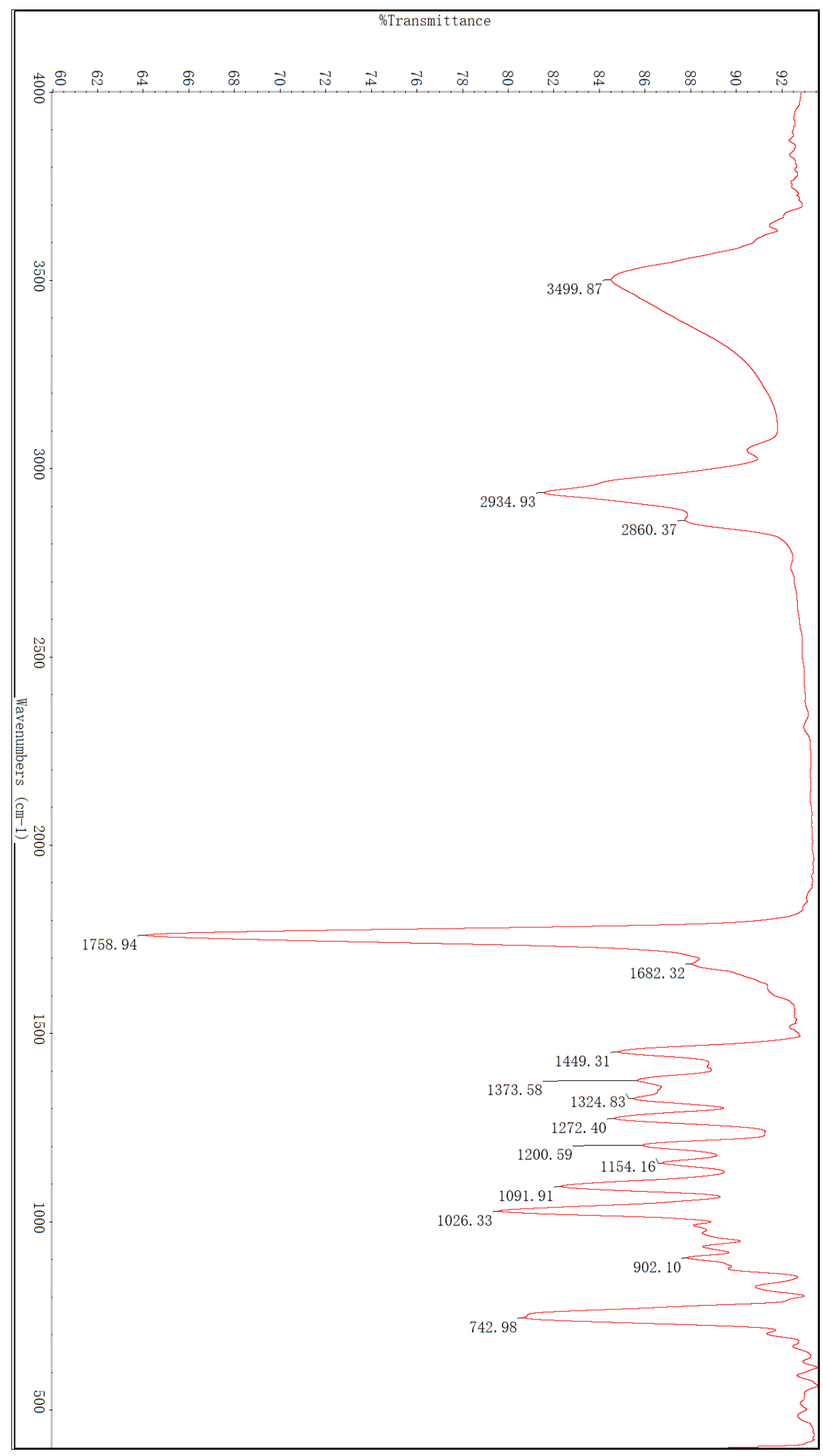


Figure S22. ${ }^{1} \mathrm{H}$ NMR spectrum $(500 \mathrm{MHz})$ of horienoid B (2) in methanol- $d_{4}$.

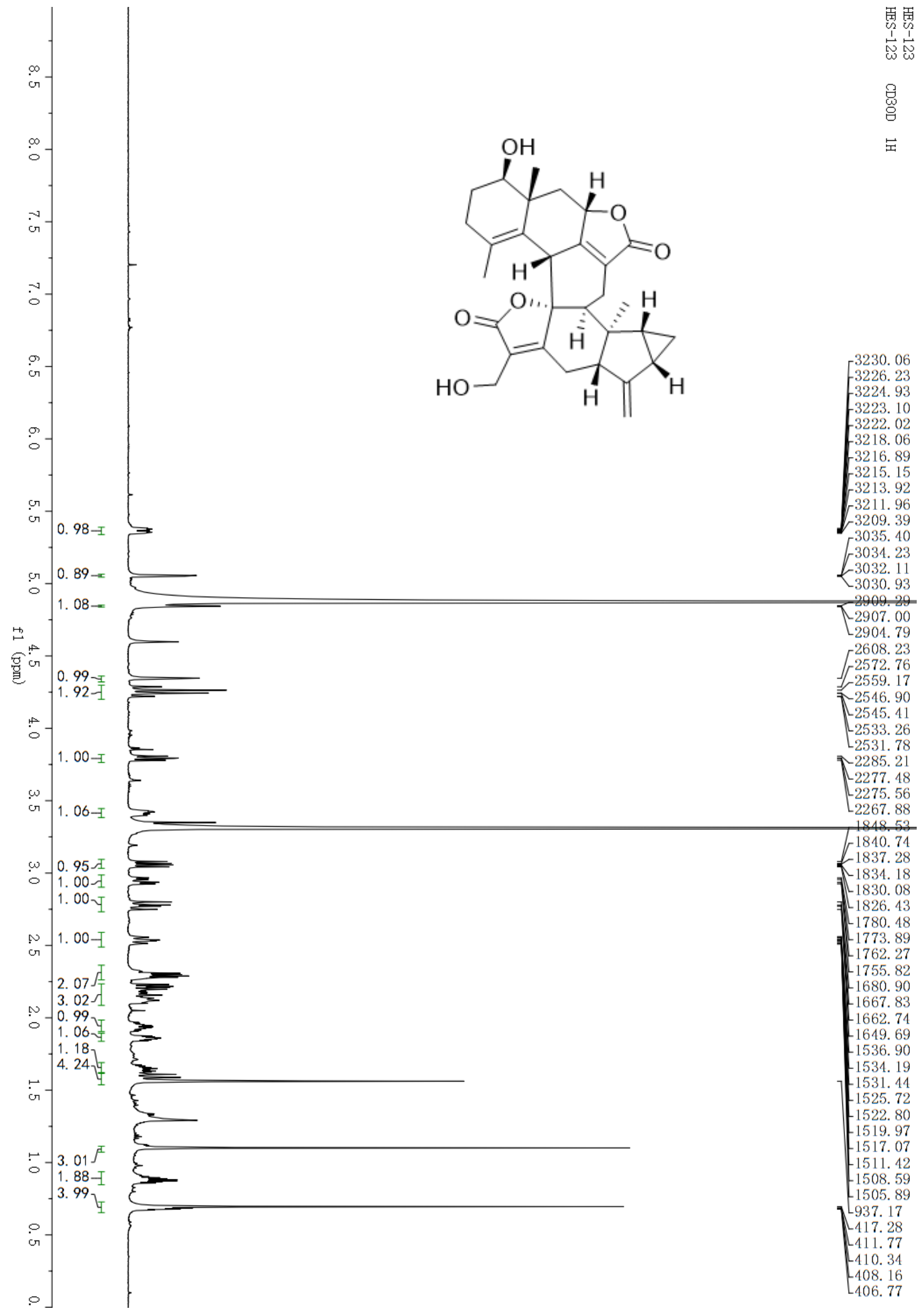


Figure S23. ${ }^{13} \mathrm{C}\left\{{ }^{1} \mathrm{H}\right\}$ NMR spectrum $(125 \mathrm{MHz})$ of horienoid B (2) in methanol- $d_{4}$.

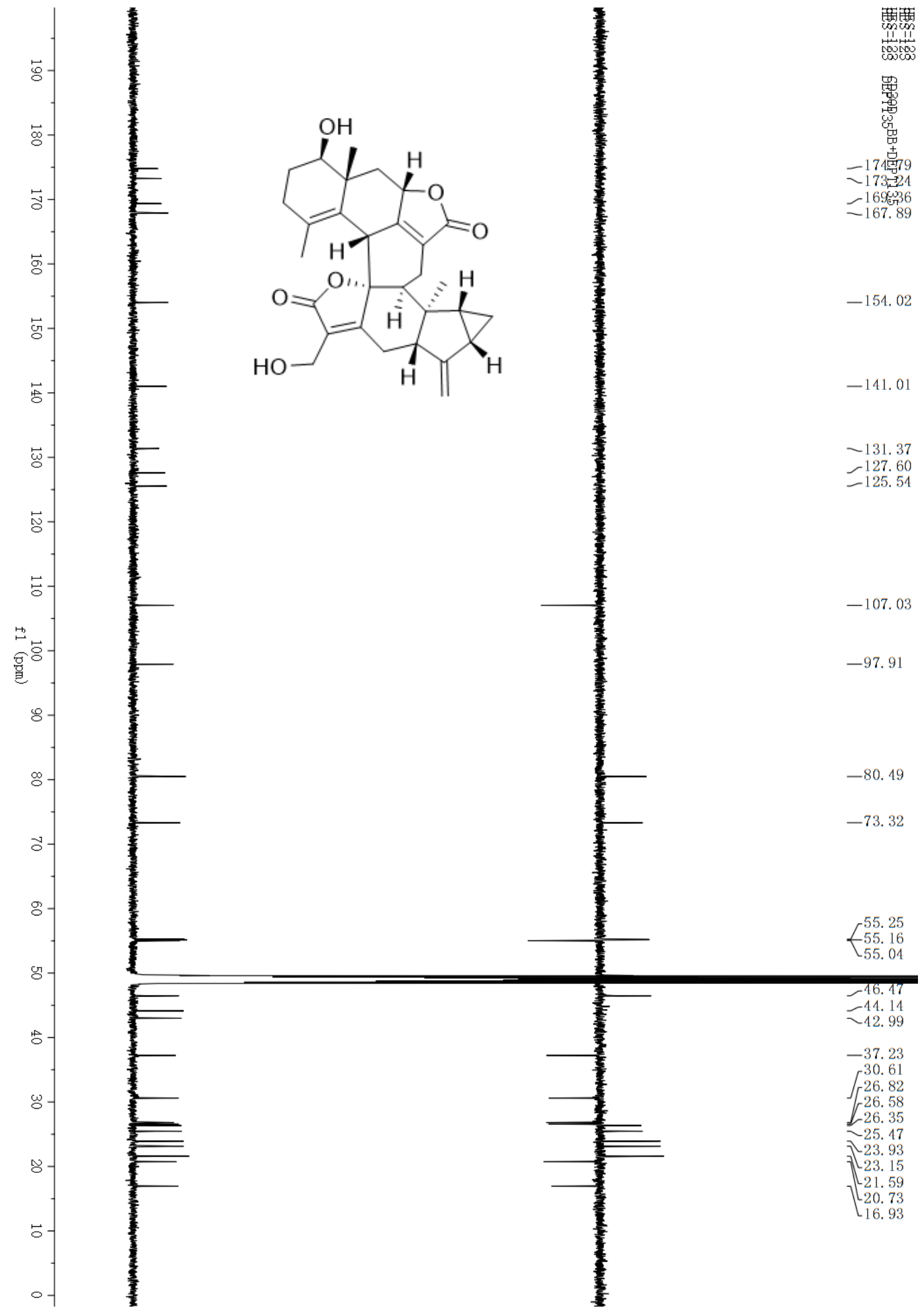


Figure S24. ${ }^{1} \mathrm{H}-{ }^{1} \mathrm{H}$ COSY spectrum $(500 \mathrm{MHz})$ of horienoid B (2) in methanol- $d_{4}$.

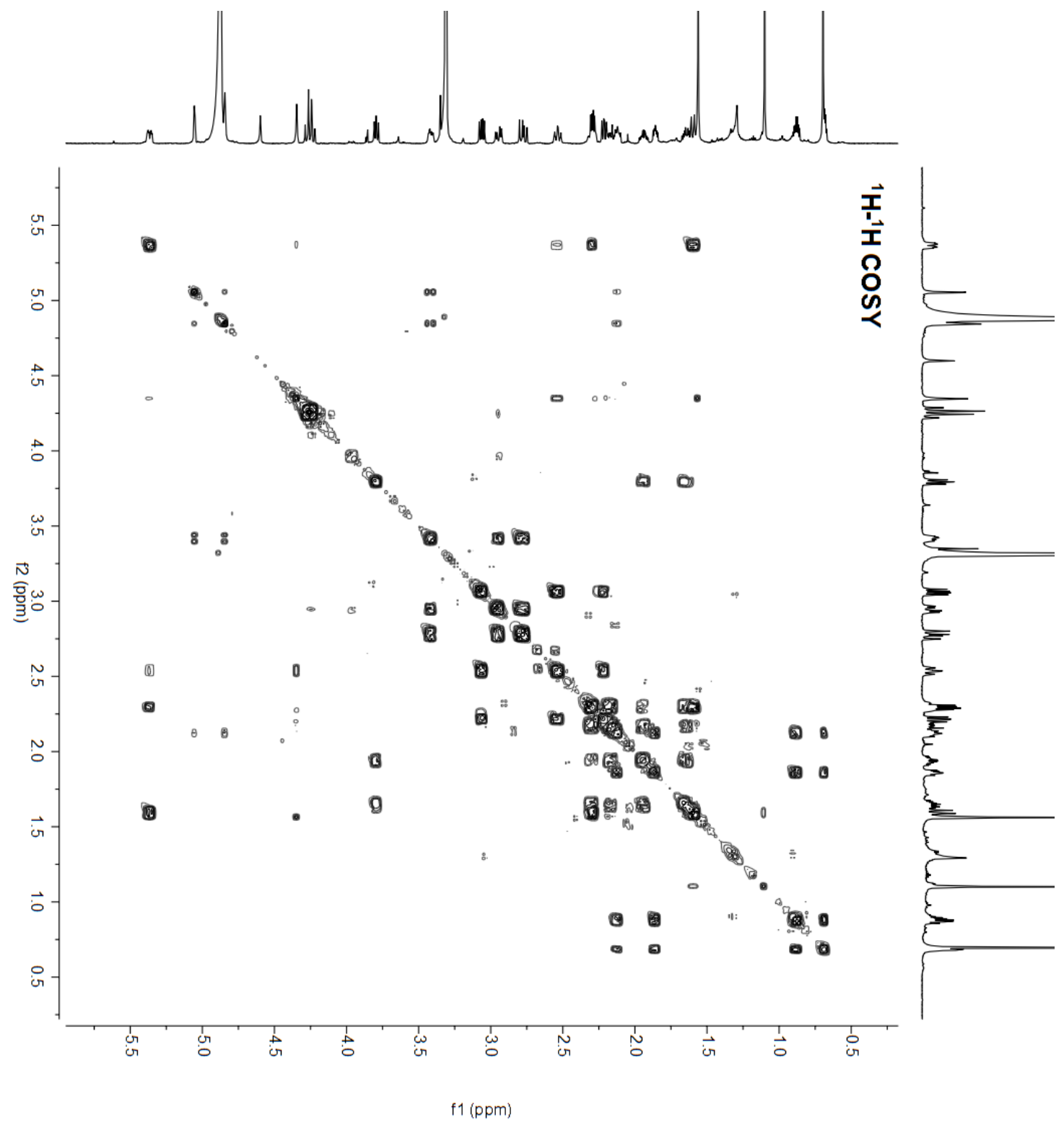


Figure S25. HSQC spectrum (500 MHz) of horienoid B (2) in methanol- $d_{4}$.

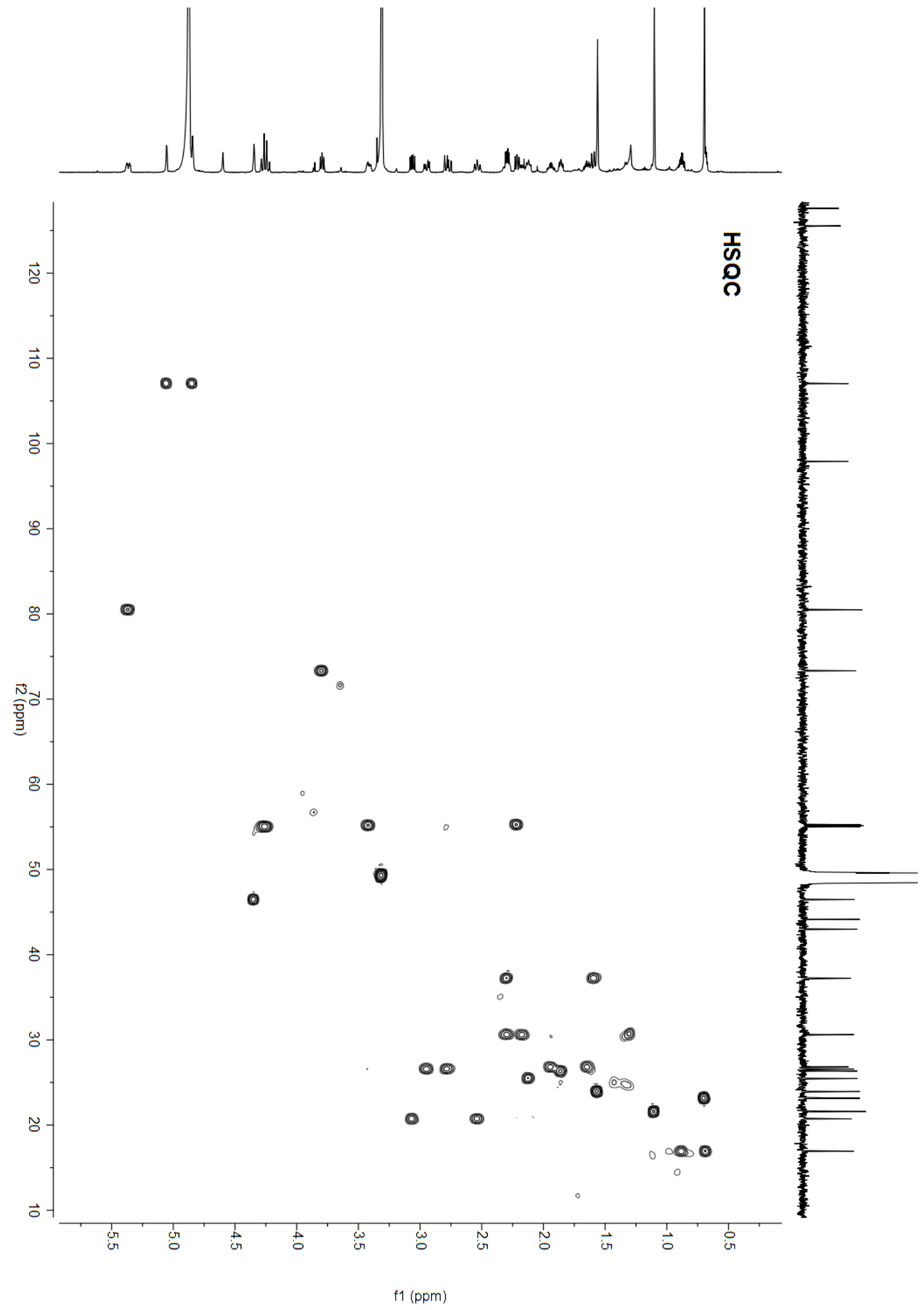


Figure S26. HMBC spectrum $(500 \mathrm{MHz})$ of horienoid B (2) in methanol- $d_{4}$.

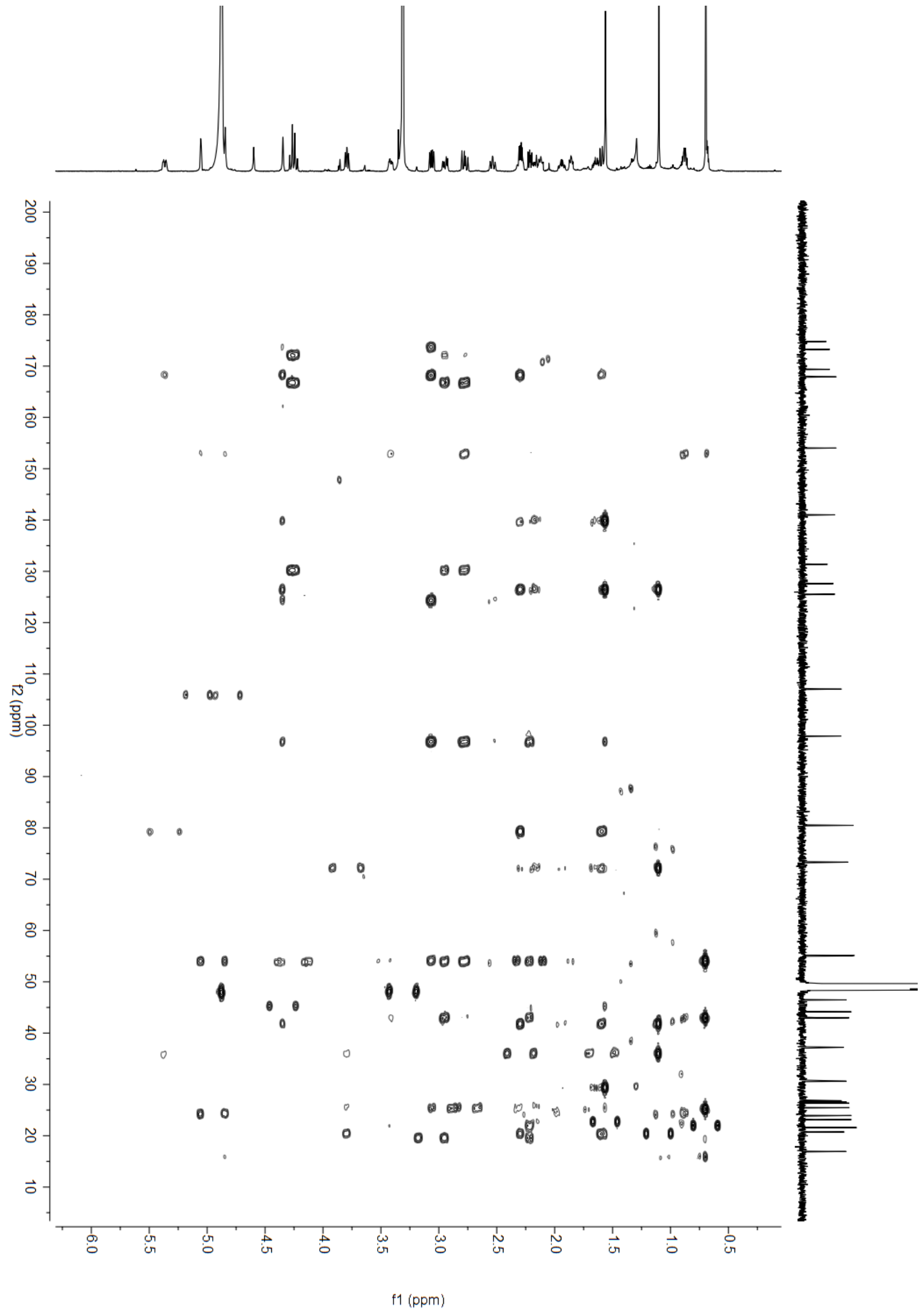


Figure S27. NOESY spectrum (500 MHz) of horienoid B (2) in methanol- $d_{4}$.

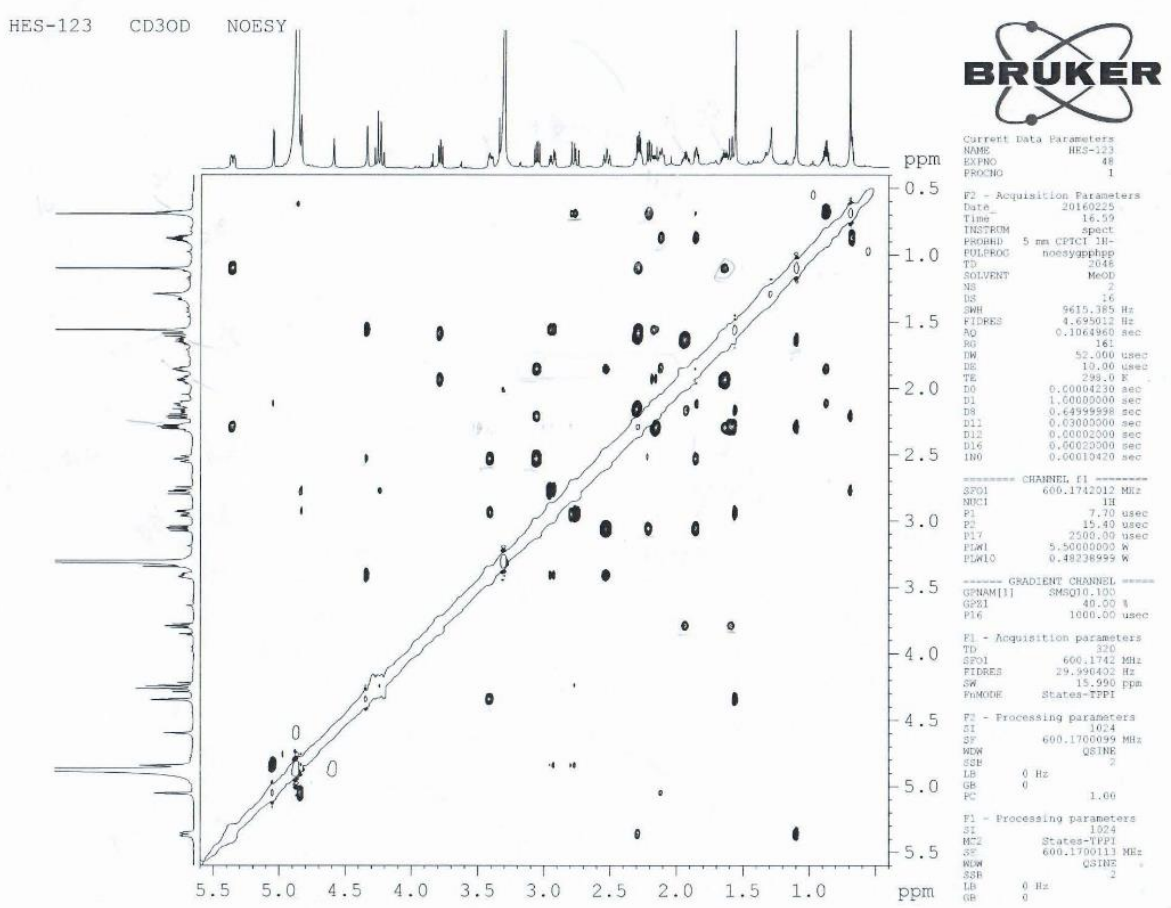


Figure S28. ESIMS spectrum of horienoid B (2).

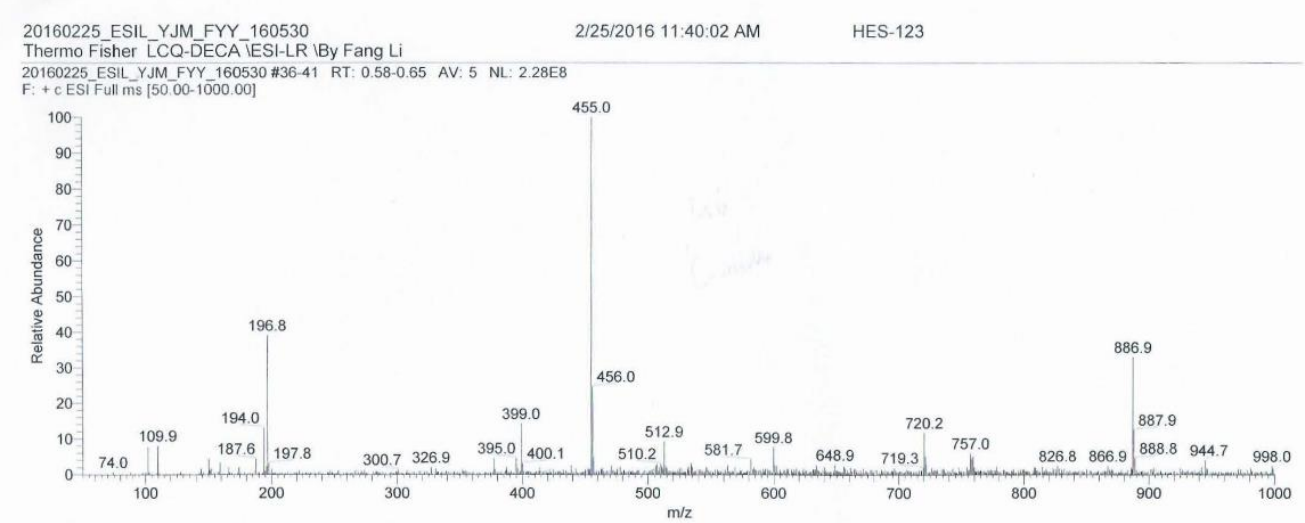

20160225 _ESIL_YJM_FYY_160530 \#36 RT: 0.55 AV: 1 NL: $6.83 E 6$
F:-cESI Full ms [50.00-1000.00]

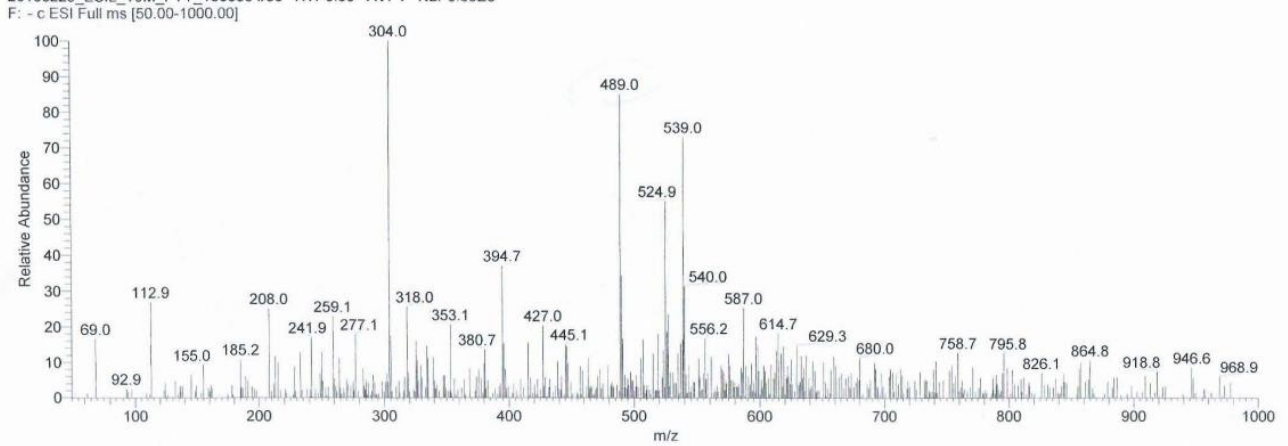


Figure S29. (+)-HRESIMS spectrum of horienoid B (2).

\section{Elemental Composition Report}

Page 1

Single Mass Analysis

Tolerance $=5.0$ PPM / DBE: $\min =-1.5, \max =50.0$

Element prediction: Off

Number of isotope peaks used for $\mathrm{i}-\mathrm{FIT}=3$

Monoisotopic Mass, Even Electron lons

200 formula(e) evaluated with 1 results within limits (up to 50 closest results for each mass) Elements Used:

$\begin{array}{llll}C: & \text { H: }-80 & -120 & \text { O: } 0-20 \quad \mathrm{Na}: 0-1\end{array}$

C: 120

LCT PXE KE324

26-Feb-2016

HES-123_20160226 32 (0.687) AM2 (Ar, 10000.0,0.00,1.00); ABS; Cm (17:35)

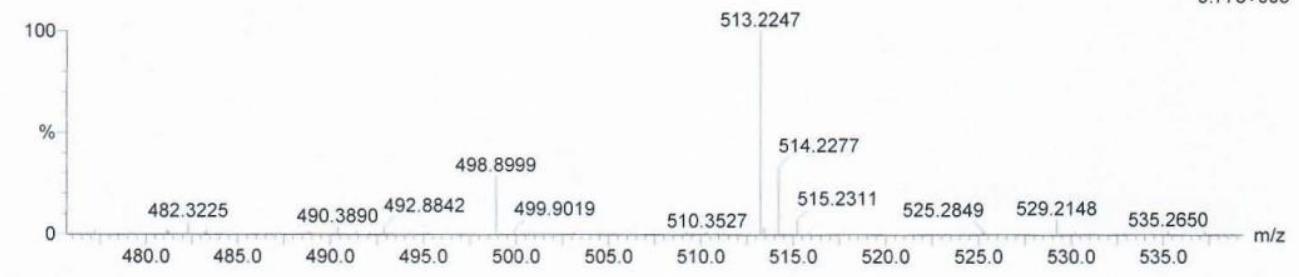

Maximum:

Mass Calc. Mass mDa PPM DBE

i-EIT (Norm) Formula

513.2247

513.2253

$-0.6$

$-1.2$

13.5

120.6

0.0

C30 H34 O6 Na 
Figure S30. IR spectrum of horienoid B (2).

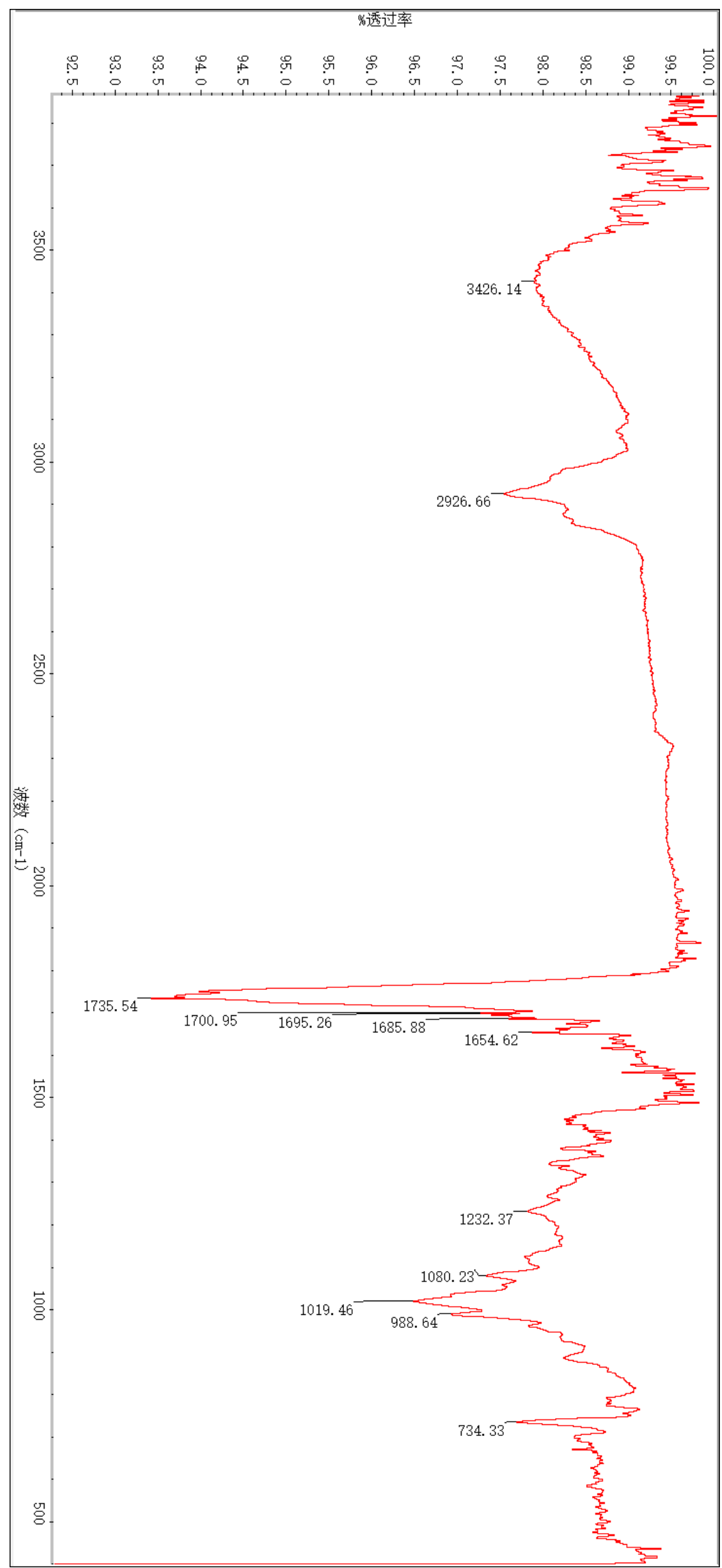

